\title{
Hydricity-promoted [1,5]-H Shifts in Acetalic Ketenimines and Carbodiimides
}

\author{
Mateo Alajarín,* Baltasar Bonillo, María-Mar Ortín, Pilar Sánchez-Andrada, \\ Ángel Vidal \\ Departamento de Química Orgánica, Facultad de Química, Universidad de Murcia, Campus de \\ Espinardo, 30100, Murcia, Spain
}

\section{Table of Contents}

General Methods and Materials

Preparation of 2-azidobenzaldehyde ethylene acetals 6a-d

Preparation of 2-azidobenzaldehyde ethylene dithioacetals $\mathbf{6 e , f .}$

Preparation of 2-(triphenylphosphoranylideneamino)benzaldehyde ethylene acetals 7a-d and 2-(triphenylphosphoranylideneamino)benzaldehyde ethylene dithioacetals 7 e,f.

Preparation of ketenimine 1a.

Preparation of 4,4-ethylenedioxy-3,4-dihydroquinolines 3a-e.

Preparation of 4,4-ethylenedithio-3,4-dihydroquinolines $\mathbf{3 f - h}$

Preparation of carbodiimides 21a-f.

Preparation of 3-aryl-4,4-ethylenedioxy-3,4-dihydroquinazolines 22a-f..

TABLE S1. Electronic energy including the zero point vibrational correction, relative energy and first frequency of the stationary points found in the transformations $9 \rightarrow \mathbf{1 1}, \mathbf{1 2} \rightarrow \mathbf{1 4}$, and $\mathbf{1 5} \rightarrow \mathbf{1 7} \ldots$

Selected Interaction from the Second Order Perturbation Analysis.

Figure S1. B3LYP/6-31+G**-optimized geometries of stationary points found in the transformation $\mathbf{9} \rightarrow \mathbf{1 1 \ldots}$ S16

Figure S2. B3LYP/6-31+G**-optimized geometries of stationary points found in the transformation $\mathbf{1 2} \rightarrow \mathbf{1 4}$.. $\quad \mathrm{S} 17$

Figure S3. B3LYP/6-31+G**-optimized geometries of stationary points found in the transformation $\mathbf{1 5} \rightarrow \mathbf{1 7}$..

Cartesian coordinates of the stationary points found in the transformations $\mathbf{9} \rightarrow \mathbf{1 1}, \mathbf{1 2} \rightarrow \mathbf{1 4}$, and $\mathbf{1 5} \rightarrow \mathbf{1 7}$ optimized at the B3LYP/6-31+G** theoretical level.

References. 


\section{General Methods}

All melting points are uncorrected. Infrared (IR) spectra were recorded neat or as Nujol emulsions.

${ }^{1} \mathrm{H}$ NMR spectra were recorded in $\mathrm{CDCl}_{3}$ at 300 or $400 \mathrm{MHz} .{ }^{13} \mathrm{C}$ NMR spectra were recorded in $\mathrm{CDCl}_{3}$ at 75 or $100 \mathrm{MHz} .{ }^{31} \mathrm{P} \mathrm{NMR}$ spectra were recorded in $\mathrm{CDCl}_{3}$ at $121.4 \mathrm{MHZ}$, using $\mathrm{H}_{3} \mathrm{PO}_{4}$ as internal reference. The chemical shifts are expressed in $\mathrm{ppm}$, relative to $\mathrm{Me}_{4} \mathrm{Si}$ at $\delta=0.00 \mathrm{ppm}$ for ${ }^{1} \mathrm{H}$, while the chemical shifts for ${ }^{13} \mathrm{C}$ are reported relative to the resonance of $\mathrm{CDCl}_{3} \delta=77.10 \mathrm{ppm}$. Mass spectra were determined at an ionizing voltage of $70 \mathrm{eV}$.

Materials: $\quad$ 2-Azidobenzaldehyde $\quad \mathbf{5 a},{ }^{1} \quad$ 2-azido-5-methylbenzaldehyde $\quad \mathbf{5 b}{ }^{2} \quad$ 2-azido-5chlorobenzaldehyde $\mathbf{5 c}{ }^{3} \quad$ 2-azido-3-methylbenzaldehyde $\quad \mathbf{5 d}^{2}{ }^{2}$ diphenylketene $^{4}$ and methylphenylketene ${ }^{5}$ were prepared by published procedures.

\section{Preparation of 2-azidobenzaldehyde ethylene acetals 6a-d $(X=0)$}

To a solution of the corresponding 2-azidobenzaldehyde $5(15 \mathrm{mmol})$ in benzene $(75 \mathrm{~mL})$ ethylene glycol $(1.12 \mathrm{~g}, 18 \mathrm{mmol})$ and $p$-toluenesulfonic acid monohydrate $(0.1 \mathrm{~g})$ were added. The mixture was heated at reflux temperature for 3 hours, with azeotropic removing of water by using a DeanStark. After cooling at room temperature, diethyl ether $(50 \mathrm{~mL})$ was added and the resulting mixture was washed with saturated sodium hydrogen carbonate solution $(3 \times 50 \mathrm{~mL})$ and brine $(3 \times 50 \mathrm{~mL})$. The organic phase was dried over anhydrous magnesium sulfate. The solvent was removed under reduced pressure and the resulting material was purified by column chromatography on silica gel, using hexanes/diethyl ether (4:1, v/v) as eluent.

2-Azidobenzaldehyde ethylene acetal 6a: yield 91\%; colorless oil; IR (neat) 2133 (vs), 1593 (s), 1490 (s), 1455 (s), 1398 (s), 1299 (vs), 1210 (m), 1116 (vs), 1073 (vs), 974 (s), 945 (s), 760 (vs), $685(\mathrm{~m}) \mathrm{cm}^{-1} ;{ }^{1} \mathrm{H}$ NMR $\left(\mathrm{CDCl}_{3}, 400 \mathrm{MHz}\right) \delta$ 4.00-4.07 (m, $\left.2 \mathrm{H}\right), 4.09-4.17$ (m, $\left.2 \mathrm{H}\right), 6.02$ (s, $\left.1 \mathrm{H}\right)$, 7.14-7.19 (m, $2 \mathrm{H}), 7.37-7.41(\mathrm{~m}, 1 \mathrm{H}), 7.57-7.59(\mathrm{~m}, 1 \mathrm{H}) ;{ }^{13} \mathrm{C} \mathrm{NMR}\left(\mathrm{CDCl}_{3}, 100 \mathrm{MHz}\right) \delta 65.5$, 99.4, 118.3, 124.8, 127.4, 128.7 (s), 130.5, 138.4 (s); MS (EI, 70 eV) m/z (rel int) $191\left(\mathrm{M}^{+}, 10\right), 119$ (100). Anal. Calcd for $\mathrm{C}_{9} \mathrm{H}_{9} \mathrm{~N}_{3} \mathrm{O}_{2}$ (191.19): C, 56.54; H, 4.74; N, 21.98. Found: C, 56.57; H, 4.59; N, 22.01. 
2-Azido-5-methylbenzaldehyde ethylene acetal 6b: yield 90\%; colorless oil; IR (neat) 2134 (vs), 1594 (m), 1497 (s), 1303 (vs), 1174 (s), 1123 (vs), 1075 (vs), 967 (s), 948 (s), 892 (w), 816 (m) cm ${ }^{1} ;{ }^{1} \mathrm{H}$ NMR $\left(\mathrm{CDCl}_{3}, 400 \mathrm{MHz}\right) \delta 2.33(\mathrm{~s}, 3 \mathrm{H}), 3.97-4.08(\mathrm{~m}, 2 \mathrm{H}), 4.08-4.18(\mathrm{~m}, 2 \mathrm{H}), 5.98(\mathrm{~s}, 1 \mathrm{H})$, $7.06(\mathrm{~d}, 1 \mathrm{H}, J=8.1 \mathrm{~Hz}), 7.17-7.21(\mathrm{~m}, 1 \mathrm{H}), 7.38-7.39(\mathrm{~m}, 1 \mathrm{H}) ;{ }^{13} \mathrm{C} \mathrm{NMR}\left(\mathrm{CDCl}_{3}, 100 \mathrm{MHz}\right) \delta$ 20.9, 65.4, 99.4, 118.2, 127.8, 128.3 (s), 131.1, 134.6 (s), 135.7 (s); MS (EI, 70 eV) m/z (rel int) 205 $\left(\mathrm{M}^{+}, 12\right), 133$ (100). Anal. Calcd for $\mathrm{C}_{10} \mathrm{H}_{11} \mathrm{~N}_{3} \mathrm{O}_{2}$ (205.22): C, 58.53; H, 5.40; N, 20.48. Found: C, 58.34; H, 5.31; N, 20.29.

2-Azido-5-chlorobenzaldehyde ethylene acetal 6c: yield $92 \%$; mp 55-57 ${ }^{\circ} \mathrm{C}$ (colorless prisms, $n$ pentane); IR (nujol) 2135 (vs), 2098 (vs), 1585 (m), 1485 (vs), 1301 (vs), 1203 (m), 1106 (vs), 1070 (vs), 1029 (m), 974 (s), 945 (s), 892 (m), 817 (s) cm ${ }^{-1} ;{ }^{1} \mathrm{H} \mathrm{NMR}\left(\mathrm{CDCl}_{3}, 400 \mathrm{MHz}\right) \delta$ 3.97-4.05 (m, $2 \mathrm{H}), 4.07-4.14(\mathrm{~m}, 2 \mathrm{H}), 5.96(\mathrm{~s}, 1 \mathrm{H}), 7.08$ (d, $1 \mathrm{H}, J=8.5 \mathrm{~Hz}), 7.33$ (dd, $1 \mathrm{H}, J=8.5,2.5 \mathrm{~Hz})$, $7.54(\mathrm{~d}, 1 \mathrm{H}, J=2.5 \mathrm{~Hz}) ;{ }^{13} \mathrm{C} \mathrm{NMR}\left(\mathrm{CDCl}_{3}, 100 \mathrm{MHz}\right) \delta 65.5,98.9,119.7,127.8,130.3,130.5$ (s), 130.9 (s), 137.2 (s); MS (EI, $70 \mathrm{eV}) \mathrm{m} / \mathrm{z}$ (rel int) $227\left(\mathrm{M}^{+}+2,2\right), 225\left(\mathrm{M}^{+}, 5\right), 153$ (100). Anal. Calcd for $\mathrm{C}_{9} \mathrm{H}_{8} \mathrm{ClN}_{3} \mathrm{O}_{2}$ (225.63): C, 47.91; H, 3.57; N, 18.62. Found: C, 47.78; H, 3.39; N, 18.45 .

2-Azido-3-methylbenzaldehyde ethylene acetal 6d: yield 81\%; colorless oil; IR (neat) 2107 (vs), 1595 (w), 1471 (s), 1444 (m), 1390 (m), 1296 (s), 1111 (vs), 1023 (m), 969 (m), 953 (m), 788 (m) $\mathrm{cm}^{-1}$; ${ }^{1} \mathrm{H}$ NMR $\left(\mathrm{CDCl}_{3}, 400 \mathrm{MHz}\right) \delta 2.41(\mathrm{~s}, 3 \mathrm{H}), 4.01-4.09(\mathrm{~m}, 2 \mathrm{H}), 4.09-4.17(\mathrm{~m}, 2 \mathrm{H}), 6.10(\mathrm{~s}, 1$ $\mathrm{H}), 7.12(\mathrm{t}, 1 \mathrm{H}, J=7.6 \mathrm{~Hz}), 7.17-7.19(\mathrm{~m}, 1 \mathrm{H}), 7.41-7.43(\mathrm{~m}, 1 \mathrm{H}) ;{ }^{13} \mathrm{C} \mathrm{NMR}\left(\mathrm{CDCl}_{3}, 100 \mathrm{MHz}\right)$ $\delta$ 18.0, 65.4, 100.6, 125.0, 125.9, 131.5 (s), 132.2, 132.9 (s), 136.8 (s); MS (EI, 70 eV) m/z (rel int) $205\left(\mathrm{M}^{+}, 7\right), 104$ (100). Anal. Calcd for $\mathrm{C}_{10} \mathrm{H}_{11} \mathrm{~N}_{3} \mathrm{O}_{2}$ (205.22): C, 58.53; H, 5.40; N, 20.48. Found: C, 58.38; H, 5.29; N, 20.24.

\section{Preparation of 2-azidobenzaldehyde ethylene dithioacetals $6 e, f(X=S)$}

To a solution of the corresponding 2-azidobenzaldehyde $5(10 \mathrm{mmol})$ in anhydrous dichloromethane $(40 \mathrm{~mL})$ 1,2-ethanedithiol $(1.41 \mathrm{~g}, 15 \mathrm{mmol})$ was added. Next, and under nitrogen, boron trifluoride diethyl etherate $(0.25 \mathrm{ml})$ was added, and the reaction mixture was stirred at room temperature for 4 hours. Then $5 \% \mathrm{NaOH}$ solution $(50 \mathrm{~mL})$ was added. The organic layer was separated, washed with water $(2 \times 40 \mathrm{~mL})$ and dried over anhydrous magnesium sulfate. The solvent was removed under reduced pressure, and the residue was purified by column chromatography on silica gel, using hexanes/diethyl ether $(9: 1 ; \mathrm{v} / \mathrm{v})$ as eluent. 
2-Azidobenzaldehyde ethylene dithioacetal 6e: yield $84 \%$; mp $82-83^{\circ} \mathrm{C}$ (colorless prisms, $n$ pentane); IR (nujol) 2141 (vs), 1574 (s), 1295 (vs), 1083 (m), 1041 (w), 944 (w), 850 (m), 755 (vs), $698(\mathrm{~m}) \mathrm{cm}^{-1} ;{ }^{1} \mathrm{H}$ NMR $\left(\mathrm{CDCl}_{3}, 400 \mathrm{MHz}\right) \delta 3.29-3.37$ (m, $\left.2 \mathrm{H}\right), 3.40-3.47$ (m, $\left.2 \mathrm{H}\right), 5.92$ (s, $\left.1 \mathrm{H}\right)$, 7.10-7.15 (m, $2 \mathrm{H}), 7.29(\mathrm{td}, 1 \mathrm{H}, J=7.7,1.6 \mathrm{~Hz}), 7.77(\mathrm{dd}, 1 \mathrm{H}, J=7.7,1.6 \mathrm{~Hz}) ;{ }^{13} \mathrm{C} \mathrm{NMR}$ $\left(\mathrm{CDCl}_{3}, 100 \mathrm{MHz}\right) \delta 39.8,49.8,118.0,125.0,129.0,132.1$ (s), $137.6(\mathrm{~s})$; MS (EI, $\left.70 \mathrm{eV}\right) \mathrm{m} / \mathrm{z}$ (rel int) $223\left(\mathrm{M}^{+}, 6\right), 167$ (100). Anal. Calcd for $\mathrm{C}_{9} \mathrm{H}_{9} \mathrm{~N}_{3} \mathrm{~S}_{2}$ (223.32): C, 48.40; H, 4.06; N, 18.82. Found: C, 48.21; H, 3.88; N, 18.80 .

2-Azido-5-chlorobenzaldehyde ethylene dithioacetal 6f: yield $86 \%$; $\mathrm{mp} 76^{\circ} \mathrm{C}$ (colorless prisms, n-pentane); IR (nujol) 2131 (vs), 2082 (vs), 1290 (vs), 1230 (w), 1181 (m), 1153 (w), 1117 (s), 902 (w), 817 (vs) cm ${ }^{-1}$; ${ }^{1} \mathrm{H}$ NMR $\left(\mathrm{CDCl}_{3}, 400 \mathrm{MHz}\right) \delta 3.29-3.36$ (m, $\left.2 \mathrm{H}\right), 3.39-3.46$ (m, $\left.2 \mathrm{H}\right), 5.83$ (s, 1 $\mathrm{H}), 7.03(\mathrm{~d}, 1 \mathrm{H}, J=8.5 \mathrm{~Hz}), 7.24(\mathrm{dd}, 1 \mathrm{H}, J=8.5,2.5 \mathrm{~Hz}), 7.74(\mathrm{~d}, 1 \mathrm{H}, J=2.5 \mathrm{~Hz}) ;{ }^{13} \mathrm{C} \mathrm{NMR}$ $\left(\mathrm{CDCl}_{3}, 100 \mathrm{MHz}\right) \delta 39.8,49.3,119.3,128.9,129.1,130.4$ (s), 134.4 (s), 136.2 (s); MS (EI, $\left.70 \mathrm{eV}\right)$ $\mathrm{m} / \mathrm{z}$ (rel int) $259\left(\mathrm{M}^{+}+2,2\right), 257\left(\mathrm{M}^{+}, 7\right), 169$ (100). Anal. Calcd for $\mathrm{C}_{9} \mathrm{H}_{8} \mathrm{ClN}_{3} \mathrm{~S}_{2}$ (257.77): C, 41.94; H, 3.13; N, 16.30. Found: C, 41.77; H, 3.08; N, 16.44.

Preparation of 2-(triphenylphosphoranylideneamino)benzaldehyde ethylene acetals 7a-d and 2-(triphenylphosphoranylideneamino)benzaldehyde ethylene dithioacetals 7e,f

To a solution of the corresponding 2-azidobenzaldehyde ethylene acetal 6a-d (4 mmol) or 2azidobenzaldehyde ethylene dithioacetal 6e,f $(4 \mathrm{mmol})$ in anhydrous diethyl ether $(40 \mathrm{~mL})$ triphenylphosphane (1.05 g, $4 \mathrm{mmol}$ ) was added in five portions. The reaction mixture was stirred at room temperature under nitrogen for 16 hours. Then, the precipitated compounds 7 were isolated by filtration.

These compounds were used in the following step without further purification. For analytical samples compounds 7 were recrystallized from diethyl ether.

2-(Triphenylphosphoranylideneamino)benzaldehyde ethylene acetal 7a: yield 98\%; $\mathrm{mp} 182$ $184^{\circ} \mathrm{C}$ (colorless prisms, diethyl ether); IR (nujol) 1595 (s), 1456 (vs), 1439 (vs), 1341 (vs), 1112 (vs), 1061 (vs), 1028 (m), 964 (m), 944 (m), 763 (s), 747 (m), 721 (vs), 701 (vs) cm ${ }^{-1}$; ${ }^{1} \mathrm{H}$ NMR $\left(\mathrm{CDCl}_{3}, 400 \mathrm{MHz}\right) \delta 3.97-4.05$ (m, $\left.2 \mathrm{H}\right), 4.10-4.18$ (m, $\left.2 \mathrm{H}\right), 6.41$ (dt, $\left.1 \mathrm{H}, J=8.0,1.2 \mathrm{~Hz}\right), 6.63-$ $6.67(\mathrm{~m}, 1 \mathrm{H}), 6.66(\mathrm{~s}, 1 \mathrm{H}), 6.80-6.85(\mathrm{~m}, 1 \mathrm{H}), 7.38-7.50(\mathrm{~m}, 10 \mathrm{H}), 7.73-7.79(\mathrm{~m}, 6 \mathrm{H}) ;{ }^{13} \mathrm{C} \mathrm{NMR}$ $\left(\mathrm{CDCl}_{3}, 100 \mathrm{MHz}\right) \delta 65.2,101.6,117.3,121.6(\mathrm{~d}, J=9.7 \mathrm{~Hz}), 126.5(\mathrm{~d}, J=1.9 \mathrm{~Hz}), 128.5(\mathrm{~d}, J=$ $12.0 \mathrm{~Hz}), 128.9,131.1$ (d, $J=20.2 \mathrm{~Hz})(\mathrm{s}), 131.6(\mathrm{~d}, J=2.7 \mathrm{~Hz}), 131.9$ (d, $J=100.0 \mathrm{~Hz})(\mathrm{s}), 132.7$ 
(d, $J=9.6 \mathrm{~Hz}), 150.0(\mathrm{~s}) ;{ }^{31} \mathrm{P} \mathrm{NMR}\left(\mathrm{CDCl}_{3}, \mathrm{H}_{3} \mathrm{PO}_{4}, 121.4 \mathrm{MHz}\right) \delta 1.4 ; \mathrm{MS}(\mathrm{EI}, 70 \mathrm{eV}) \mathrm{m} / \mathrm{z}$ (rel int) $425\left(\mathrm{M}^{+}, 7\right), 352$ (100). Anal. Calcd for $\mathrm{C}_{27} \mathrm{H}_{24} \mathrm{NO}_{2} \mathrm{P}$ (425.47): C, 76.22; H, 5.69; N, 3.29. Found: C, 76.01; H, 5.58; N, 3.45.

5-Methyl-2-(triphenylphosphoranylideneamino)benzaldehyde ethylene acetal 7b: yield 90\%; mp 176- $177^{\circ} \mathrm{C}$ (colorless prisms, diethyl ether); IR (nujol) 1613 (s), 1493 (vs), 1437 (vs), 1368 (vs), 1312 (m), 1280 (m), 1164 (s), 1113 (vs), 1071 (vs), 967 (s), 895 (m), 826 (m), 759 (m), 724 (vs), 701 (s) $\mathrm{cm}^{-1} ;{ }^{1} \mathrm{H} \mathrm{NMR}\left(\mathrm{CDCl}_{3}, 300 \mathrm{MHz}\right) \delta 2.18$ (s, $\left.3 \mathrm{H}\right), 3.98-4.08$ (m, $\left.2 \mathrm{H}\right), 4.12-4.22(\mathrm{~m}, 2 \mathrm{H})$, 6.34 (dd, $1 \mathrm{H}, J=8.0,1.3 \mathrm{~Hz}), 6.64-6.67$ (m, $2 \mathrm{H}), 7.27$ (t, $1 \mathrm{H}, J=2.3 \mathrm{~Hz}), 7.37-7.48$ (m, $9 \mathrm{H})$, 7.72-7.79 (m, $6 \mathrm{H}) ;{ }^{13} \mathrm{C} \mathrm{NMR}\left(\mathrm{CDCl}_{3}, 75 \mathrm{MHz}\right) \delta 20.7,65.1,101.4,121.4(\mathrm{~d}, J=9.7 \mathrm{~Hz}), 126.3$ (s), $126.9(\mathrm{~d}, J=1.7 \mathrm{~Hz}), 128.5(\mathrm{~d}, J=12.0 \mathrm{~Hz}), 129.7,130.3(\mathrm{~d}, J=20.4 \mathrm{~Hz})(\mathrm{s}), 131.5(\mathrm{~d}, J=2.8$ $\mathrm{Hz}), 131.7$ (d, $J=99.6 \mathrm{~Hz})(\mathrm{s}), 132.6$ (d, $J=9.6 \mathrm{~Hz}), 147.3$ (s) ; ${ }^{31} \mathrm{P}$ NMR $\left(\mathrm{CDCl}_{3}, \mathrm{H}_{3} \mathrm{PO}_{4}, 121.4\right.$ $\mathrm{MHz}) \delta 1.2$; MS (EI, $70 \mathrm{eV}) \mathrm{m} / \mathrm{z}$ (rel int) $439\left(\mathrm{M}^{+}, 7\right), 366$ (100). Anal. Calcd for $\mathrm{C}_{28} \mathrm{H}_{26} \mathrm{NO}_{2} \mathrm{P}$ (439.49): C, 76.52; H, 5.96; N, 3.19. Found: C, 76.41; H, 5.78; N, 3.25.

5-Chloro-2-(triphenylphosphoranylideneamino)benzaldehyde ethylene acetal 7c: yield 92\%; $\mathrm{mp} 162-163^{\circ} \mathrm{C}$ (colorless prisms, diethyl ether); IR (nujol) 1589 (s), 1435 (vs), 1415 (vs), 1329 (vs), 1258 (m), 1185 (m), 1106 (vs), 1064 (vs), 1023 (s), 964 (s), 883 (m), 816 (m), 780 (w), 747 (m), 721 (s), 696 (vs) cm ${ }^{-1} ;{ }^{1} \mathrm{H}$ NMR $\left(\mathrm{CDCl}_{3}, 400 \mathrm{MHz}\right) \delta$ 3.99-4.07 (m, $\left.2 \mathrm{H}\right), 4.11-4.19$ (m, $\left.2 \mathrm{H}\right), 6.32$ (dt, $1 \mathrm{H}, J=8.5,1.4 \mathrm{~Hz}), 6.61(\mathrm{~s}, 1 \mathrm{H}), 6.77$ (dd, $1 \mathrm{H}, J=8.5,2.8 \mathrm{~Hz}), 7.39-7.45(\mathrm{~m}, 7 \mathrm{H}), 7.47-$ $7.52(\mathrm{~m}, 3 \mathrm{H}), 7.70-7.77(\mathrm{~m}, 6 \mathrm{H}) ;{ }^{13} \mathrm{C} \mathrm{NMR}\left(\mathrm{CDCl}_{3}, 100 \mathrm{MHz}\right) \delta 65.2,100.9,121.9$ (s), $122.3(\mathrm{~d}, J$ $=9.9 \mathrm{~Hz}), 126.5(\mathrm{~d}, J=1.5 \mathrm{~Hz}), 128.6(\mathrm{~d}, J=12.0 \mathrm{~Hz}), 131.1(\mathrm{~d}, J=100.2 \mathrm{~Hz})(\mathrm{s}), 131.8(\mathrm{~d}, J=$ $2.8 \mathrm{~Hz}), 132.3$ (s), 132.6 (d, $J=9.7 \mathrm{~Hz}), 148.8(\mathrm{~s}) ;{ }^{31} \mathrm{P} \mathrm{NMR}\left(\mathrm{CDCl}_{3}, \mathrm{H}_{3} \mathrm{PO}_{4}, 121.4 \mathrm{MHz}\right) \delta 2.7$; MS (EI, $70 \mathrm{eV}) \mathrm{m} / \mathrm{z}$ (rel int) $461\left(\mathrm{M}^{+}+2,2\right), 459\left(\mathrm{M}^{+}, 5\right), 386$ (100). Anal. Calcd for $\mathrm{C}_{27} \mathrm{H}_{23} \mathrm{ClNO}_{2} \mathrm{P}$ (459.91): C, 70.51; H, 5.04; N, 3.05. Found: C, 70.33; H, 5.08; N, 2.88.

3-Methyl-2-(triphenylphosphoranylideneamino)benzaldehyde ethylene acetal 7d: yield 85\%; $\mathrm{mp} 143-144^{\circ} \mathrm{C}$ (colorless prisms, diethyl ether); IR (nujol) 1597 (m), 1436 (vs), 1342 (s), 1279 (m), 1111 (vs), 1088 (vs), 947 (m), 911 (w), 814 (w), 781 (m), 758 (m), 716 (vs), 695 (vs) cm ${ }^{-1} ;{ }^{1} \mathrm{H}$ NMR $\left(\mathrm{CDCl}_{3}, 400 \mathrm{MHz}\right) \delta 1.92(\mathrm{~s}, 3 \mathrm{H}), 3.37-3.44(\mathrm{~m}, 2 \mathrm{H}), 3.82-3.91(\mathrm{~m}, 2 \mathrm{H}), 5.90$ (s, $\left.1 \mathrm{H}\right), 6.73$ (td, $1 \mathrm{H}, J=7.5,2.4 \mathrm{~Hz}), 6.96-6.99(\mathrm{~m}, 1 \mathrm{H}), 7.30-7.32$ (m, $1 \mathrm{H}), 7.36-7.41$ (m, $6 \mathrm{H})$, 7.45-7.50 (m, $3 \mathrm{H}), 7.59-7.65(\mathrm{~m}, 6 \mathrm{H}) ;{ }^{13} \mathrm{C} \mathrm{NMR}\left(\mathrm{CDCl}_{3}, 100 \mathrm{MHz}\right) \delta 20.8,64.6,101.4,119.0(\mathrm{~d}, J=3.3 \mathrm{~Hz})$, $123.5(\mathrm{~d}, J=2.7 \mathrm{~Hz}), 128.3(\mathrm{~d}, J=11.9 \mathrm{~Hz}), 130.7$ (d, $J=2.7 \mathrm{~Hz}), 131.2$ (d, $J=2.7 \mathrm{~Hz}), 131.5$ (d, $J$ $=7.4 \mathrm{~Hz})(\mathrm{s}), 132.4(\mathrm{~d}, J=9.6 \mathrm{~Hz}), 132.7$ (d, $J=101.9 \mathrm{~Hz})(\mathrm{s}), 133.7$ (d, $J=6.1 \mathrm{~Hz})(\mathrm{s}), 147.6(\mathrm{~s})$; 
${ }^{31} \mathrm{P}$ NMR $\left(\mathrm{CDCl}_{3}, \mathrm{H}_{3} \mathrm{PO}_{4}, 121.4 \mathrm{MHz}\right) \delta 2.9$; MS (EI, $\left.70 \mathrm{eV}\right) \mathrm{m} / \mathrm{z}\left(\right.$ rel int) $439\left(\mathrm{M}^{+}, 5\right), 366(100)$. Anal. Calcd for $\mathrm{C}_{28} \mathrm{H}_{26} \mathrm{NO}_{2} \mathrm{P}$ (439.49): C, 76.52; H, 5.96; N, 3.19. Found: C, 76.31; H, 5.78; N, 3.05 .

2-(Triphenylphosphoranylideneamino)benzaldehyde ethylene dithioacetal 7e: yield 93\%; IR (nujol) 1588 (s), 1444 (vs), 1188 (m), 1162 (m), 1109 (vs), 1060 (m), 1031 (m), 1002 (m), 851 (w), 756 (s), 722 (vs), 696 (vs) cm ${ }^{-1} ;{ }^{1} \mathrm{H} \mathrm{NMR}\left(\mathrm{CDCl}_{3}, 400 \mathrm{MHz}\right) \delta$ 3.27-3.34 (m, $\left.2 \mathrm{H}\right)$, 3.35-3.42 (m, 2 $\mathrm{H}), 6.38(\mathrm{dt}, 1 \mathrm{H}, J=8.0,1.1 \mathrm{~Hz}), 6.62-6.66(\mathrm{~m}, 2 \mathrm{H}), 6.76(\mathrm{td}, 1 \mathrm{H}, J=7.4,1.7 \mathrm{~Hz}), 7.41-7.45(\mathrm{~m}$, $6 \mathrm{H}), 7.47-7.52(\mathrm{~m}, 3 \mathrm{H}), 7.66(\mathrm{dt}, 1 \mathrm{H}, J=7.5,2.1 \mathrm{~Hz}), 7.76-7.82(\mathrm{~m}, 6 \mathrm{H}) ;{ }^{13} \mathrm{C} \mathrm{NMR}\left(\mathrm{CDCl}_{3}, 100\right.$ $\mathrm{MHz}) \delta 39.1,52.5,117.0,120.6(\mathrm{~d}, J=10.3 \mathrm{~Hz}), 126.5(\mathrm{~d}, J=2.2 \mathrm{~Hz}), 127.5,128.6(\mathrm{~d}, J=12.2$ Hz), $131.1(\mathrm{~d}, J=99.8 \mathrm{~Hz})(\mathrm{s}), 131.7$ (d, $J=2.8 \mathrm{~Hz}), 132.7(\mathrm{~d}, J=9.7 \mathrm{~Hz}), 135.2(\mathrm{~d}, J=22.1 \mathrm{~Hz})$ (s), 149.0 (s); ${ }^{31} \mathrm{P} \mathrm{NMR}\left(\mathrm{CDCl}_{3}, \mathrm{H}_{3} \mathrm{PO}_{4}, 121.4 \mathrm{MHz}\right) \delta 2.3$; MS (EI, $\left.70 \mathrm{eV}\right) \mathrm{m} / \mathrm{z}$ (rel int) $457\left(\mathrm{M}^{+}\right.$, 15), 262 (100).

5-Chloro-2-(triphenylphosphoranylideneamino)benzaldehyde ethylene dithioacetal 7f: yield 90\%; mp 176-177 $\mathrm{C}$ (colorless prisms, diethyl ether); IR (nujol) 1582 (m), 1436 (vs), 1408 (m), 1278 (m), 1113 (vs), 1027 (m), 1005 (w), 895 (m), 813 (m), 755 (m), 793 (m), 725 (vs), 697 (s) cm ${ }^{1} ;{ }^{1} \mathrm{H} \mathrm{NMR}\left(\mathrm{CDCl}_{3}, 400 \mathrm{MHz}\right) \delta 3.27-3.39$ (m, $\left.4 \mathrm{H}\right), 6.26(\mathrm{dd}, 1 \mathrm{H}, J=8.4,1.2 \mathrm{~Hz}), 6.51(\mathrm{~s}, 1 \mathrm{H})$, $6.70(\mathrm{dd}, 1 \mathrm{H}, J=8.4,2.7 \mathrm{~Hz}), 7.42-7.46(\mathrm{~m}, 6 \mathrm{H}), 7.49-7.54(\mathrm{~m}, 3 \mathrm{H}), 7.62(\mathrm{t}, 1 \mathrm{H}, J=2.7 \mathrm{~Hz})$, 7.73-7.79 (m, $6 \mathrm{H}) ;{ }^{13} \mathrm{C} \mathrm{NMR}\left(\mathrm{CDCl}_{3}, 100 \mathrm{MHz}\right) \delta 39.1,52.0,121.2(\mathrm{~d}, J=10.3 \mathrm{~Hz}), 121.7$ (s), $126.6(\mathrm{~d}, J=2.0 \mathrm{~Hz}), 127.0,128.7$ (d, $J=12.1 \mathrm{~Hz}), 130.7$ (d, $J=100.1 \mathrm{~Hz})(\mathrm{s}), 131.9$ (d, $J=2.8$ $\mathrm{Hz}), 132.6$ (d, $J=9.8 \mathrm{~Hz}), 137.2$ (d, $J=22.2 \mathrm{~Hz})(\mathrm{s}), 147.7(\mathrm{~s}) ;{ }^{31} \mathrm{P} \mathrm{NMR}\left(\mathrm{CDCl}_{3}, \mathrm{H}_{3} \mathrm{PO}_{4}, 121.4\right.$ $\mathrm{MHz}) \delta 4.1$; MS (EI, $70 \mathrm{eV}) \mathrm{m} / \mathrm{z}$ (rel int) $493\left(\mathrm{M}^{+}+2,5\right), 491\left(\mathrm{M}^{+}, 11\right), 262$ (100). Anal. Calcd for $\mathrm{C}_{27} \mathrm{H}_{23} \mathrm{ClNPS}_{2}$ (492.04): C, 65.91; H, 4.71; N, 2.85. Found: C, 65.73; H, 4.55; N, 2.77.

\section{Preparation of ketenimine 1a}

To a solution of 2-(triphenylphosphoranylideneamino)benzaldehyde ethylene acetal 7a (0.42 g, 1 $\mathrm{mmol})$ in anhydrous dichloromethane $(25 \mathrm{~mL})$ a solution of diphenylketene $(0.19 \mathrm{~g}, 1 \mathrm{mmol})$ in the same solvent $(5 \mathrm{~mL})$ was added. After stirring at room temperature for $30 \mathrm{~min}$ the solvent was removed under reduced pressure, and the resulting material was chromatographed on a silica gel column using hexanes/diethyl ether $(4: 1, \mathrm{v} / \mathrm{v})$ as eluent.

Ketenimine 1a: yield 87\%; mp 94-95 C (yellow prisms, diethyl ether/n-pentane); IR (nujol) 1995 (vs), 1592 (s), 1493 (s), 1278 (m), 1209 (m), 1178 (w), 1093 (s), 1076 (vs), 979 (s), 952 (s), 939 (s), 
764 (vs), 694 (vs), 642 (s) cm ${ }^{-1} ;{ }^{1} \mathrm{H}$ NMR $\left(\mathrm{CDCl}_{3}, 300 \mathrm{MHz}\right) \delta$ 3.96-4.06 (m, $\left.2 \mathrm{H}\right), 4.09-4.19$ (m, 2 $\mathrm{H}), 6.31(\mathrm{~s}, 1 \mathrm{H}), 7.21-7.30(\mathrm{~m}, 2 \mathrm{H}), 7.31-7.37(\mathrm{~m}, 11 \mathrm{H}), 7.64-7.67(\mathrm{~m}, 1 \mathrm{H}) ;{ }^{13} \mathrm{C} \mathrm{NMR}\left(\mathrm{CDCl}_{3}\right.$, $75 \mathrm{MHz})$ \&: 65.6, 77.8 (s), 99.9, 122.7, 126.5, 127.2, 127.7, 128.0, 128.9, 130.3, 132.3 (s), 133.9 (s), 139.4 (s), 190.8 (s). Anal. Calcd for $\mathrm{C}_{23} \mathrm{H}_{19} \mathrm{NO}_{2}$ (341.41): C, 80.92; H, 5.61; N, 4.10. Found: C, $80.78 ; \mathrm{H}, 5.51 ; \mathrm{N}, 4.02$.

\section{Preparation of 4,4-ethylenedioxy-3,4-dihydroquinolines 3a-e}

To a solution of the corresponding 2-(triphenylphosphoranylideneamino)benzaldehyde ethylene acetal $7(1 \mathrm{mmol})$ in anhydrous toluene $(15 \mathrm{~mL})$ methylphenylketene $(0.13 \mathrm{~g}, 1 \mathrm{mmol})$ or diphenylketene $(0.19 \mathrm{~g}, 1 \mathrm{mmol})$ in the same solvent $(5 \mathrm{ml})$ was added. The reaction mixture was stirred at room temperature for $15 \mathrm{~min}$, and the formation of the corresponding ketenimine 1 was established by IR spectroscopy (the IR spectra of the reaction mixtures showed strong absorptions around $2000 \mathrm{~cm}^{-1}$, characteristic of the $\mathrm{N}=\mathrm{C}=\mathrm{C}$ ). Next, the solutions containing ketenimines 1 were heated at reflux temperature up to the total disappearance of the cumulenic band in their IR spectra, approximately $1 \mathrm{~h}$. After cooling, the solvent was removed under reduced pressure. The resulting material was purified by column chromatography on silica gel, using hexanes/diethyl ether (7:3, $\mathrm{v} / \mathrm{v})$ as eluent.

4,4-Ethylenedioxy-3,3-diphenyl-3,4-dihydroquinoline 3a: yield 70\%; mp 184-185 ${ }^{\circ} \mathrm{C}$ (colorless prisms, diethyl ether); IR (nujol) 1619 (m), 1597 (m), 1496 (s), 1278 (w), 1250 (s), 1159 (s), 1093 (m), 1066 (s), 1036 (s), 1000 (m), 949 (s), 897 (w), 768 (vs), 708 (vs) cm ${ }^{-1} ;{ }^{1} \mathrm{H} \mathrm{NMR}_{\text {(CDCl }}, 400$ MHz) $\delta 3.15(\mathrm{t}, 2 \mathrm{H}, J=6.9 \mathrm{~Hz}), 3.85(\mathrm{t}, 2 \mathrm{H}, J=6.9 \mathrm{~Hz}), 7.19-7.23(\mathrm{~m}, 6 \mathrm{H}), 7.31-7.36(\mathrm{~m}, 7 \mathrm{H})$, 7.56-7.58 (m, $1 \mathrm{H}), 8.61(\mathrm{~s}, 1 \mathrm{H}) ;{ }^{13} \mathrm{C} \mathrm{NMR}\left(\mathrm{CDCl}_{3}, 100 \mathrm{MHz}\right) \delta 59.0(\mathrm{~s}), 65.7,107.9(\mathrm{~s}), 122.6$, $127.2,127.7,127.8,128.4,129.5,130.4,131.1$ (s), 140.4 (s), 142.6 (s), 167.5; MS (EI, $70 \mathrm{eV}) \mathrm{m} / \mathrm{z}$ (rel int) $341\left(\mathrm{M}^{+}, 77\right), 165$ (100). Anal. Calcd for $\mathrm{C}_{23} \mathrm{H}_{19} \mathrm{NO}_{2}$ (341.41): C, 80.92; H, 5.61; N, 4.10. Found: C, 80.78; H, 5.39; N, 4.22.

4,4-Ethylenedioxy-3-methyl-3-phenyl-3,4-dihydroquinoline $3 \mathrm{~b}$ : yield $58 \%$; $\mathrm{mp} \quad 120-121^{\circ} \mathrm{C}$ (colorless prisms, diethyl ether); IR (nujol) 1623 (vs), 1601 (s), 1496 (s), 1279 (s), 1226 (vs), 1145 (vs), 1084 (vs), 1036 (s), 1009 (s), 965 (s), 938 (vs), 902 (m), 853 (w), 824 (w), 768 (vs), 751 (s), 707 (vs) $\mathrm{cm}^{-1} ;{ }^{1} \mathrm{H} \mathrm{NMR}\left(\mathrm{CDCl}_{3}, 400 \mathrm{MHz}\right) \delta 1.53$ (s, $\left.3 \mathrm{H}\right), 3.15$ (dt, $\left.1 \mathrm{H}, J=7.7,6.2 \mathrm{~Hz}\right), 3.50$ (dt, $1 \mathrm{H}, J=7.4,6.3 \mathrm{~Hz}), 3.73(\mathrm{dt}, 1 \mathrm{H}, J=7.7,6.2 \mathrm{~Hz}), 3.97$ (dt, $1 \mathrm{H}, J=7.4,6.3 \mathrm{~Hz}), 7.25-7.42$ (m, 6 $\mathrm{H}), 7.46-7.50(\mathrm{~m}, 3 \mathrm{H}), 8.15(\mathrm{~s}, 1 \mathrm{H}) ;{ }^{13} \mathrm{C} \mathrm{NMR}\left(\mathrm{CDCl}_{3}, 100 \mathrm{MHz}\right) \delta 17.5,50.4(\mathrm{~s}), 65.0,66.7$, 108.6 (s), 123.5, 127.5, 127.6, 128.0, 128.2, 129.2, 129.4 (s), 129.7, 138.8 (s), 142.7 (s), 170.2; MS 
(EI, $70 \mathrm{eV}) \mathrm{m} / \mathrm{z}$ (rel int) $279\left(\mathrm{M}^{+}, 100\right)$. Anal. Calcd for $\mathrm{C}_{18} \mathrm{H}_{17} \mathrm{NO}_{2}$ (279.34): C, 77.40; H, 6.13; N, 5.01. Found: C, 77.20; H, 5.97; N, 4.88.

4,4-Ethylenedioxy-6-methyl-3,3-diphenyl-3,4-dihydroquinoline $3 \mathrm{c}$ : yield $68 \%$; $\mathrm{mp} 180-181^{\circ} \mathrm{C}$ (colorless prisms, diethyl ether); IR (nujol) 1621 (s), 1599 (s), 1287 (s), 1263 (s), 1201 (s), 1173 (vs), 1093 (s), 1067 (vs), 1038 (s), 1004 (s), 948 (s), 896 (w), 848 (m), 822 (m), 764 (vs), 703 (vs) $\mathrm{cm}^{-1} ;{ }^{1} \mathrm{H} \mathrm{NMR}\left(\mathrm{CDCl}_{3}, 400 \mathrm{MHz}\right) \delta 2.42(\mathrm{~s}, 3 \mathrm{H}), 3.12(\mathrm{t}, 2 \mathrm{H}, J=6.7 \mathrm{~Hz}), 3.84(\mathrm{t}, 2 \mathrm{H}, J=6.7$ $\mathrm{Hz}), 7.12(\mathrm{dd}, 1 \mathrm{H}, J=7.9,1.3 \mathrm{~Hz}), 7.19-7.22(\mathrm{~m}, 7 \mathrm{H}), 7.31-7.36(\mathrm{~m}, 5 \mathrm{H}), 8.53(\mathrm{~s}, 1 \mathrm{H}) ;{ }^{13} \mathrm{C} \mathrm{NMR}$ $\left(\mathrm{CDCl}_{3}, 100 \mathrm{MHz}\right) \delta 21.7,59.1$ (s), 65.7, 108.1 (s), 123.2, 127.1, 127.7, 127.8, 130.0, 130.4, 130.8 (s), 138.4 (s), 140.4 (s), 140.6 (s), 166.3; MS (EI, 70 eV) m/z (rel int) 355 (M+100). Anal. Calcd for $\mathrm{C}_{24} \mathrm{H}_{21} \mathrm{NO}_{2}$ (355.44): C, 81.10; H, 5.96; N, 3.94. Found: C, 80.93; H, 5.79; N, 3.85.

6-Chloro-4,4-ethylenedioxy-3,3-diphenyl-3,4-dihydroquinoline 3d: yield 68\%; mp 197-198 ${ }^{\circ} \mathrm{C}$ (colorless prisms, diethyl ether); IR (nujol) 1617 (s), 1414 (s), 1252 (vs), 1192 (s), 1157 (vs), 1101 (vs), 1086 (s), 1066 (vs), 1041 (vs), 1003 (s), 952 (vs), 898 (s), 827 (s), 769 (vs), 713 (vs), 641 (s) $\mathrm{cm}^{-1} ;{ }^{1} \mathrm{H} \mathrm{NMR}\left(\mathrm{CDCl}_{3}, 400 \mathrm{MHz}\right) \delta 3.14(\mathrm{t}, 2 \mathrm{H}, J=6.9 \mathrm{~Hz}), 3.84(\mathrm{t}, 2 \mathrm{H}, J=6.9 \mathrm{~Hz}), 7.21-7.27$ $(\mathrm{m}, 8 \mathrm{H}), 7.28-7.34(\mathrm{~m}, 4 \mathrm{H}), 7.54(\mathrm{~d}, 1 \mathrm{H}, J=2.2 \mathrm{~Hz}), 8.60(\mathrm{~s}, 1 \mathrm{H}) ;{ }^{13} \mathrm{C} \mathrm{NMR}\left(\mathrm{CDCl}_{3}, 100 \mathrm{MHz}\right)$ $\delta 58.8$ (s), 65.8, 107.6 (s), 123.0, 127.4, 127.8, 129.2, 129.5, 130.3 (s), 132.8 (s), 133.6 (s), 140.0 (s), 141.2 (s), 168.0; MS (EI, $70 \mathrm{eV}) \mathrm{m} / \mathrm{z}$ (rel int) $377\left(\mathrm{M}^{+}+2,26\right), 375\left(\mathrm{M}^{+}, 74\right), 165$ (100). Anal. Calcd for $\mathrm{C}_{23} \mathrm{H}_{18} \mathrm{ClNO}_{2}$ (375.85): C, 73.50; H, 4.83; N, 3.73. Found: C, 73.29; H, 4.73; N, 3.69.

4,4-Ethylenedioxy-8-methyl-3,3-diphenyl-3,4-dihydroquinoline $3 \mathrm{e}$ : yield $67 \%$; $\mathrm{mp}$ 199-200 ${ }^{\circ} \mathrm{C}$ (colorless prisms, diethyl ether); IR (nujol) 1598 (m), 1582 (m), 1498 (s), 1278 (s), 1171 (vs), 1121 (m), 1057 (vs), 1037 (s), 1023 (s), 957 (s), 849 (m), 795 (s), 763 (vs), 705 (vs) cm ${ }^{-1} ;{ }^{1} \mathrm{H}$ NMR $\left(\mathrm{CDCl}_{3}, 400 \mathrm{MHz}\right) \delta 2.38(\mathrm{~s}, 3 \mathrm{H}), 3.13(\mathrm{t}, 2 \mathrm{H}, J=6.7 \mathrm{~Hz}), 3.82(\mathrm{t}, 2 \mathrm{H}, J=6.7 \mathrm{~Hz}), 7.14-7.23(\mathrm{~m}$, $8 \mathrm{H}), 7.32-7.36(\mathrm{~m}, 4 \mathrm{H}), 7.40(\mathrm{dd}, 1 \mathrm{H}, J=7.2,1.8 \mathrm{~Hz}), 8.64(\mathrm{~s}, 1 \mathrm{H}) ;{ }^{13} \mathrm{C} \mathrm{NMR}\left(\mathrm{CDCl}_{3}, 100\right.$ MHz) $\delta 17.6,58.5$ (s), 65.7, 108.3 (s), 120.3, 127.1, 127.7, 127.9, 130.4, 130.9 (s), 131.4, 136.0 (s), 140.6 (s), 140.7 (s), 166.0; MS (EI, $70 \mathrm{eV}) \mathrm{m} / \mathrm{z}$ (rel int) $355\left(\mathrm{M}^{+}, 100\right)$. Anal. Calcd for $\mathrm{C}_{24} \mathrm{H}_{21} \mathrm{NO}_{2}$ (355.44): C, 81.10; H, 5.96; N, 3.94. Found: C, 80.91; H, 5.79; N, 3.77.

\section{Preparation of 4,4-ethylenedithio-3,4-dihydroquinolines 3f-h}

To a solution of the corresponding 2-(triphenylphosphoranylideneamino)benzaldehyde ethylene dithioacetal $7(1 \mathrm{mmol})$ in anhydrous toluene $(15 \mathrm{~mL})$ methylphenylketene $(0.13 \mathrm{~g}, 1 \mathrm{mmol})$ or 
diphenylketene $(0.19 \mathrm{~g}, 1 \mathrm{mmol})$ in the same solvent $(5 \mathrm{ml})$ was added. The reaction mixture was stirred at room temperature for $15 \mathrm{~min}$, and the formation of the corresponding ketenimine $\mathbf{1}$ was established by IR spectroscopy (the IR spectra of the reaction mixtures showed strong absorptions around $2000 \mathrm{~cm}^{-1}$, characteristic of the $\mathrm{N}=\mathrm{C}=\mathrm{C}$ ). Next, the solutions containing ketenimines 1 were heated at reflux temperature up to the total disappearance of the cumulenic band in their IR spectra, approximately $16 \mathrm{~h}$. After cooling, the solvent was removed under reduced pressure. The resulting material was purified by column chromatography on silica gel, using hexanes/diethyl ether (7:3, $\mathrm{v} / \mathrm{v})$ as eluent.

4,4-Ethylenedithio-3,3-diphenyl-3,4-dihydroquinoline 3f: yield 74\%; mp 229-230 ${ }^{\circ} \mathrm{C}$ (colorless prisms, diethyl ether); IR (nujol) 1620 (s), 1591 (m), 1283 (m), 1239 (w), 1196 (w), 1108 (m), 1038

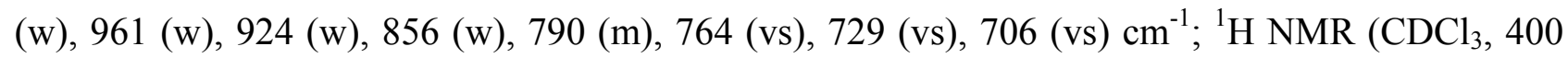
MHz) $\delta 1.28-1.34(\mathrm{~m}, 1 \mathrm{H}), 2.72-2.77(\mathrm{~m}, 1 \mathrm{H}), 2.96-3.00(\mathrm{~m}, 1 \mathrm{H}), 3.30-3.36(\mathrm{~m}, 1 \mathrm{H})$, 7.07-7.13 (m, $5 \mathrm{H}), 7.23$ (dd, $1 \mathrm{H}, J=7.8,1.6 \mathrm{~Hz}), 7.27$ (td, $1 \mathrm{H}, J=7.2,1.4 \mathrm{~Hz}), 7.28-7.33(\mathrm{~m}, 3 \mathrm{H}), 7.37$ $(\mathrm{td}, 1 \mathrm{H}, J=7.5,1.6 \mathrm{~Hz}), 7.60(\mathrm{~d}, 2 \mathrm{H}, J=6.7 \mathrm{~Hz}), 8.21(\mathrm{dd}, 1 \mathrm{H}, J=7.8,1.4 \mathrm{~Hz}), 8.51(\mathrm{~s}, 1 \mathrm{H}){ }^{13} \mathrm{C}$ $\mathrm{NMR}\left(\mathrm{CDCl}_{3}, 100 \mathrm{MHz}\right) \delta 38.9,41.9,61.4$ (s), 74.2 (s), 125.6, 126.5, 127.2, 127.6, 127.9, 128.0, 128.5, 129.1, 129.3, 133.1, 133.4 (s), 140.5 (s), 140.8 (s), 141.4 (s), 165.7; MS (EI, 70 eV) m/z (rel int) $373\left(\mathrm{M}^{+}, 51\right), 312$ (100). Anal. Calcd for $\mathrm{C}_{23} \mathrm{H}_{19} \mathrm{NS}_{2}$ (373.54): C, 73.95; H, 5.13; N, 3.75. Found: C, 73.71; H, 5.02; N, 3.64.

4,4-Ethylenedithio-3-methyl-3-phenyl-3,4-dihydroquinoline $3 \mathrm{~g}$ : yield $52 \%$; $\mathrm{mp} \quad 118-119^{\circ} \mathrm{C}$ (colorless prisms, diethyl ether); IR (nujol) 1626 (vs), 1598 (m), 1275 (m), 1243 (m), 1199 (w), 1163 (w), 1113 (w), 1079 (w), 1027 (m), 961 (m), 901 (w), 765 (vs), 729 (s), 703 (s) cm ${ }^{-1}$; ${ }^{1} \mathrm{H}$ NMR $\left(\mathrm{CDCl}_{3}, 400 \mathrm{MHz}\right) \delta 1.69$ (s, $\left.3 \mathrm{H}\right), 2.06$ (br s, $\left.1 \mathrm{H}\right), 2.93-3.02$ (m, $\left.2 \mathrm{H}\right), 3.21-3.27$ (m, $\left.1 \mathrm{H}\right)$, 7.257.32 (m, 4 H), 7.34 (dd, $1 \mathrm{H}, J=7.4,1.5 \mathrm{~Hz}), 7.41$ (dd, $1 \mathrm{H}, J=7.6,1.5 \mathrm{~Hz}), 7.57-7.59$ (m, $2 \mathrm{H})$, $7.90(\mathrm{dd}, 1 \mathrm{H}, J=7.6,1.3 \mathrm{~Hz}), 8.18(\mathrm{~s}, 1 \mathrm{H}) ;{ }^{13} \mathrm{C} \mathrm{NMR}\left(\mathrm{CDCl}_{3}, 100 \mathrm{MHz}\right) \delta 20.2,40.3,40.7,50.8$ (s), 76.4 (s), 126.6, 127.4, 127.6, 127.7, 128.7, 128.8, 130.0, 132.4 (s), 139.0 (s), 140.9 (s), 169.6; MS (EI, $70 \mathrm{eV}) \mathrm{m} / \mathrm{z}$ (rel int) $311\left(\mathrm{M}^{+}, 34\right), 250$ (100). Anal. Calcd for $\mathrm{C}_{18} \mathrm{H}_{17} \mathrm{NS}_{2}$ (311.47): C, 69.41; H, 5.50; N, 4.50. Found: C, 69.22; H, 5.32; N, 4.64.

6-Chloro-4,4-ethylenedithio-3,3-diphenyl-3,4-dihydroquinoline $3 \mathrm{~h}$ : yield $77 \%$; $\mathrm{mp}$ 228-229 ${ }^{\circ} \mathrm{C}$ (colorless prisms, diethyl ether); IR (nujol) 1624 (s), 1499 (vs), 1282 (m), 1244 (m), 1200 (w), 1176

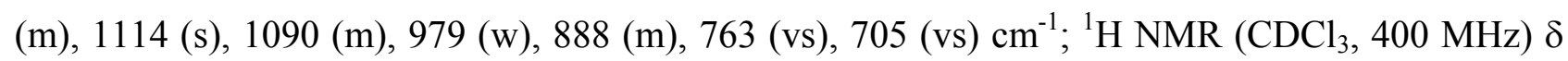
1.29-1.36 (m, $1 \mathrm{H}), 2.73-2.78$ (m, $1 \mathrm{H}), 2.95-3.00$ (m, $1 \mathrm{H}), 3.31-3.38$ (m, $1 \mathrm{H})$, 7.10-7.19 (m, $6 \mathrm{H})$, $7.23(\mathrm{dd}, 1 \mathrm{H}, J=8.3,2.3 \mathrm{~Hz}), 7.28-7.35$ (m, $3 \mathrm{H}), 7.57$ (d, $2 \mathrm{H}, J=6.7 \mathrm{~Hz}), 8.21$ (d, $1 \mathrm{H}, J=2.3$ 
$\mathrm{Hz}), 8.49$ (s, $1 \mathrm{H}) ;{ }^{13} \mathrm{C} \mathrm{NMR}\left(\mathrm{CDCl}_{3}, 100 \mathrm{MHz}\right) \delta 39.0,42.0,61.1$ (s), 73.7 (s), 125.8, 126.6, 127.4, 127.7, 128.1, 128.5, 129.0, 129.3, 133.0, 134.4 (s), 135.5 (s), 139.1 (s), 140.6 (s), 141.1 (s), 166.0; MS (EI, $70 \mathrm{eV}) \mathrm{m} / \mathrm{z}$ (rel int) $409\left(\mathrm{M}^{+}+2,24\right), 407\left(\mathrm{M}^{+}, 55\right), 346$ (100). Anal. Calcd for $\mathrm{C}_{23} \mathrm{H}_{18} \mathrm{ClNS}_{2}$ (407.99): C, 67.71; H, 4.45; N, 3.43. Found: C, 67.55; H, 4.32; N, 3.34.

\section{Preparation of carbodiimides 21 a-f}

To a solution of the corresponding 2-(triphenylphosphoranylideneamino)benzaldehyde ethylene acetal $7(\mathbf{X}=\mathbf{O})(2 \mathrm{mmol})$ in anhydrous dichloromethane $(25 \mathrm{~mL})$ a solution in the same solvent $(5$ $\mathrm{ml}$ ) of the aryl isocyanate $(2 \mathrm{mmol})$ was added. The reaction mixture was stirred at room temperature for $30 \mathrm{~min}$. The solvent was removed under reduced pressure, and the resulting material was purified by column chromatography on silica gel, using hexanes/diethyl ether (4:1, $\mathrm{v} / \mathrm{v}$ ) as eluent.

Carbodiimide 21a $\left(\mathbf{R}^{1}=\mathbf{H} ; \mathbf{R}^{2}=\mathbf{H} ; \mathbf{A r}=\mathbf{4}-\mathbf{C H}_{3} \mathbf{O}-\mathbf{C}_{6} \mathbf{H}_{4}\right)$ : yield 81\%; colorless oil; IR (neat) 2132 (vs), 1597 (s), 1579 (vs), 1519 (vs), 1491 (vs), 1458 (s), 1389 (s), 1289 (vs), 1250 (vs), 1215 (vs), 1191 (vs), 1102 (vs), 1071 (vs), 1030 (vs), 969 (s), 939 (vs), 892 (vs), 763 (vs), 737 (s) cm ${ }^{-1} ;{ }^{1} \mathrm{H}$ NMR $\left(\mathrm{CDCl}_{3}, 300 \mathrm{MHz}\right) \delta 3.77(\mathrm{~s}, 3 \mathrm{H}), 3.99-4.07$ (m, $\left.2 \mathrm{H}\right), 4.08-4.16$ (m, $\left.2 \mathrm{H}\right), 6.19(\mathrm{~s}, 1 \mathrm{H})$, 6.81-6.86 (m, 2 H), 7.09-7.14 (m, 2 H), 7.17-7.24 (m, 2 H), 7.28-7.34 (m, 1 H), 7.57 (dd, 1 H, $J=$

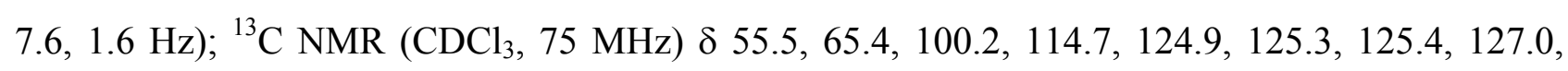
130.1, 130.6 (s), 131.3 (s), 134.7 (s), 137.6 (s), 157.5 (s); MS (EI, 70 eV) m/z (rel int) 296 (M', 35), 121 (100). Anal. Calcd for $\mathrm{C}_{17} \mathrm{H}_{16} \mathrm{~N}_{2} \mathrm{O}_{3}$ (296.32): C, 69.91; H, 5.44; N, 9.45. Found: C, 69.77; H, $5.21 ; \mathrm{N}, 9.24$.

Carbodiimide 21b $\left(\mathbf{R}^{1}=\mathbf{H} ; \mathbf{R}^{2}=\mathbf{C H}_{3} ; \mathbf{A r}=4-\mathbf{B r}-\mathbf{C}_{6} \mathbf{H}_{4}\right)$ : yield 52\%; colorless oil; IR (neat) 2130 (vs), 1583 (vs), 1511 (s), 1472 (vs), 1379 (s), 1256 (s), 1201 (vs), 1172 (s), 1105 (vs), 1069 (vs), 1009 (vs), 962 (s), 945 (s), 827 (vs), 778 (vs), 748 (s) cm ${ }^{-1} ;{ }^{1} \mathrm{H} \mathrm{NMR}\left(\mathrm{CDCl}_{3}, 400 \mathrm{MHz}\right) \delta 2.38$ (s, 3 H), 3.96-4.02 (m, 2 H), 4.06-4.12 (m, 2 H), 6.11 (s, 1 H), 7.03-7.07 (m, 2 H), 7.10 (t, 1 H, J = 7.6 $\mathrm{Hz}), 7.18-7.21(\mathrm{~m}, 1 \mathrm{H}), 7.39-7.43(\mathrm{~m}, 3 \mathrm{H}) ;{ }^{13} \mathrm{C} \mathrm{NMR}\left(\mathrm{CDCl}_{3}, 100 \mathrm{MHz}\right) \delta 18.6,65.4,101.1$, 117.9 (s), 124.7, 125.4, 125.7, 131.5, 131.9 (s), 132.6, 133.3 (s), 134.4 (s), 139.0 (s); MS (EI, 70 eV) $\mathrm{m} / \mathrm{z}$ (rel int) $360\left(\mathrm{M}^{+}+2,29\right), 358\left(\mathrm{M}^{+}, 37\right), 315$ (100). Anal. Calcd for $\mathrm{C}_{17} \mathrm{H}_{15} \mathrm{BrN}_{2} \mathrm{O}_{2}$ (359.22): C, 56.84; H, 4.21; N, 7.80. Found: C, 56.70; H, 4.07; N, 7.67.

Carbodiimide 21c $\left(\mathbf{R}^{1}=\mathbf{H} ; \mathbf{R}^{2}=\mathbf{C H}_{3} ; \mathbf{A r}=4-\mathbf{C l}-\mathbf{C}_{6} \mathbf{H}_{4}\right)$ : yield 46\%; colorless oil; IR (neat) 2144 (vs), 1591 (vs), 1573 (m), 1510 (s), 1475 (vs), 1437 (m), 1377 (s), 1255 (s), 1201 (vs), 1167 (m), 1099 (vs), 1013 (vs), 964 (s), 943 (s), 829 (vs), 778 (s), 749 (s), 659 (w), 613 (m) cm ${ }^{-1}$; ${ }^{1} \mathrm{H}$ NMR 
$\left(\mathrm{CDCl}_{3}, 400 \mathrm{MHz}\right) \delta 2.36(\mathrm{~s}, 3 \mathrm{H}), 3.92-3.98(\mathrm{~m}, 2 \mathrm{H}), 4.02-4.08$ (m, $\left.2 \mathrm{H}\right), 6.10(\mathrm{~s}, 1 \mathrm{H})$, 7.06-7.10 $(\mathrm{m}, 3 \mathrm{H}), 7.15-7.17$ (m, $1 \mathrm{H}), 7.20-7.23(\mathrm{~m}, 2 \mathrm{H}), 7.41(\mathrm{dd}, 1 \mathrm{H}, J=7.6,1.2 \mathrm{~Hz}) ;{ }^{13} \mathrm{C} \mathrm{NMR}\left(\mathrm{CDCl}_{3}\right.$, $100 \mathrm{MHz}) \delta 18.5,65.1,100.7,124.4,125.1,125.2,129.3,130.0$ (s), 131.2 (s), 131.3, 131.5 (s), 133.1 (s), 134.2 (s), 138.0 (s); MS (EI, $70 \mathrm{eV}) \mathrm{m} / \mathrm{z}$ (rel int) $316\left(\mathrm{M}^{+}+2,11\right), 314\left(\mathrm{M}^{+}, 41\right), 271$ (100). Anal. Calcd for $\mathrm{C}_{17} \mathrm{H}_{15} \mathrm{ClN}_{2} \mathrm{O}_{2}$ (314.77): C, 64.87; H, 4.80; N, 8.90. Found: C, 64.59; H, $4.68 ; \mathrm{N}, 8.82$.

Carbodiimide 21d $\left(\mathbf{R}^{1}=\mathbf{H} ; \mathbf{R}^{2}=\mathbf{C H}_{3} ; \mathbf{A r}=\mathbf{4}-\mathbf{C H}_{3}-\mathbf{C}_{6} \mathbf{H}_{4}\right)$ : yield 80\%; colorless oil; IR (neat) 2161 (vs), 1613 (w), 1597 (m), 1520 (m), 1483 (s), 1378 (m), 1278 (w), 1254 (m), 1205 (s), 1104 (s), 1020 (m), 964 (m), 945 (m), 817 (s), 785 (m), 761 (m), 717 (w) cm ${ }^{-1}$; ${ }^{1} \mathrm{~N}$ NR $\left(\mathrm{CDCl}_{3}, 400\right.$ MHz) $\delta 2.29$ (s, 3 H), 2.38 (s, 3 H), 3.94-4.00 (m, 2 H), 4.04-4.10 (m, 2 H), 6.14 (s, 1 H), 7.05-7.10 $(\mathrm{m}, 5 \mathrm{H}), 7.15-7.19(\mathrm{~m}, 1 \mathrm{H}), 7.40-7.43(\mathrm{~m}, 1 \mathrm{H}) ;{ }^{13} \mathrm{C} \mathrm{NMR}\left(\mathrm{CDCl}_{3}, 100 \mathrm{MHz}\right) \delta 18.7,20.9,65.2$, $100.8,123.8,124.4,124.9,129.9,131.3,131.4$ (s), 131.8 (s), 133.1 (s), 134.6 (s), 135.1 (s), 136.3 (s); MS (EI, $70 \mathrm{eV}) \mathrm{m} / \mathrm{z}$ (rel int) $294\left(\mathrm{M}^{+}, 33\right), 105$ (100). Anal. Calcd for $\mathrm{C}_{18} \mathrm{H}_{18} \mathrm{~N}_{2} \mathrm{O}_{2}$ (294.35): C, 73.45; H, 6.16; N, 9.52. Found: C, 73.27; H, 6.11; N, 9.36.

Carbodiimide 21e $\left(\mathbf{R}^{1}=\mathbf{C H}_{3} ; \mathbf{R}^{2}=\mathbf{H} ; \mathbf{A r}=\mathbf{4}-\mathbf{B r}-\mathbf{C}_{6} \mathbf{H}_{4}\right)$ : yield 33\%; colorless oil; IR (neat) 2146 (vs), 1581 (vs), 1515 (m), 1488 (vs), 1393 (s), 1279 (m), 1265 (m), 1234 (s), 1202 (vs), 1161 (vs),

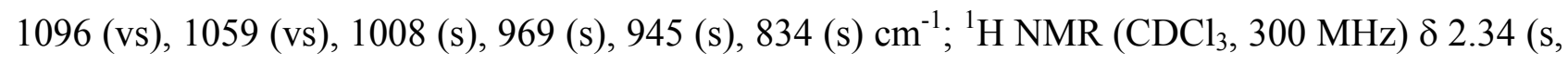
$3 \mathrm{H}), 3.98-4.07$ (m, 2 H), 4.09-4.17 (m, 2 H), 6.11 (s, 1 H), 7.02-7.06 (m, 2 H), 7.08-7.15 (m, 2 H), 7.39-7.43 (m, $3 \mathrm{H}) ;{ }^{13} \mathrm{C} \mathrm{NMR}\left(\mathrm{CDCl}_{3}, 75 \mathrm{MHz}\right) \delta 21.1,65.4,100.2,118.4(\mathrm{~s}), 125.1,125.8,127.5$, 130.9, 131.0 (s), 132.4, 133.5 (s), 134.1 (s), 135.9 (s), 138.0 (s); MS (EI, 70 eV) m/z (rel int) 360 $\left(\mathrm{M}^{+}+2,40\right), 358\left(\mathrm{M}^{+}, 43\right), 133$ (100). Anal. Calcd for $\mathrm{C}_{17} \mathrm{H}_{15} \mathrm{BrN}_{2} \mathrm{O}_{2}$ (359.22): C, 56.84; H, 4.21; N, 7.80. Found: C, 56.64; H, 4.09; N, 7.67.

Carbodiimide $21 \mathbf{f}\left(\mathbf{R}^{\mathbf{1}}=\mathbf{C H}_{3} ; \mathbf{R}^{2}=\mathbf{H} ; \mathbf{A r}=4-\mathbf{C l}-\mathbf{C}_{6} \mathbf{H}_{4}\right)$ : yield 50\%; colorless oil; IR (neat) 2143 (vs), 1587 (s), 1487 (vs), 1392 (m), 1282 (m), 1270 (m), 1205 (vs), 1164 (s), 1093 (vs), 1063 (vs), 1010 (s), 969 (s), 947 (s), 890 (m), 831 (vs) cm ${ }^{-1} ;{ }^{1} \mathrm{H}$ NMR $\left(\mathrm{CDCl}_{3}, 400 \mathrm{MHz}\right) \delta 2.34$ (s, $\left.3 \mathrm{H}\right), 4.01$ 4.07 (m, 2 H), 4.11-4.19 (m, 2 H), 6.12 (s, 1 H), 7.08-7.13 (m, 4 H), 7.25-7.29 (m, 2 H), 7.39-7.40 $(\mathrm{m}, 1 \mathrm{H}) ;{ }^{13} \mathrm{C} \mathrm{NMR}\left(\mathrm{CDCl}_{3}, 100 \mathrm{MHz}\right) \delta 21.1,65.5,100.3,125.1,125.4,127.6,129.6,130.8$ (s), 131.0, 131.1 (s), 133.6 (s), 134.3 (s), 135.9 (s), 137.5 (s); MS (EI, $70 \mathrm{eV}) \mathrm{m} / \mathrm{z}$ (rel int) $316\left(\mathrm{M}^{+}+2\right.$, 20), $314\left(\mathrm{M}^{+}, 100\right)$. Anal. Calcd for $\mathrm{C}_{17} \mathrm{H}_{15} \mathrm{ClN}_{2} \mathrm{O}_{2}$ (314.77): C, 64.87; H, 4.80; N, 8.90. Found: C, $64.76 ; \mathrm{H}, 4.71 ; \mathrm{N}, 8.78$. 


\section{Preparation of 3-aryl-4,4-ethylenedioxy-3,4-dihydroquinazolines 22a-f}

A solution of the corresponding carbodiimide $21(1 \mathrm{mmol})$ in anhydrous ortho-xylene $(25 \mathrm{~mL})$ was heated at reflux temperature for 24-36 hours. After cooling, the solvent was removed under reduced pressure. The crude material was purified by column chromatography on silica gel.

4,4-Ethylenedioxy-3-(4-methoxyphenyl)-3,4-dihydroquinazoline 22a: eluent for column chromatography: hexanes/diethyl ether $(3: 7, \mathrm{v} / \mathrm{v})$; yield $81 \% ; \mathrm{mp} 126^{\circ} \mathrm{C}$ (colorless prisms, diethyl ether); IR (nujol) 1619 (vs), 1599 (vs), 1568 (vs), 1507 (vs), 1333 (vs), 1301 (s), 1247 (vs), 1222 (vs), 1204 (s), 1181 (s), 1072 (vs), 1029 (vs), 1004 (s), 981 (vs), 951 (vs), 832 (vs), 774 (vs), 753 (s) $\mathrm{cm}^{-1}$; ${ }^{1} \mathrm{H} \mathrm{NMR}\left(\mathrm{CDCl}_{3}, 400 \mathrm{MHz}\right) \delta$ 3.57-3.63 (m, $\left.2 \mathrm{H}\right), 3.83$ (s, $\left.3 \mathrm{H}\right), 4.05-4.14$ (m, $\left.2 \mathrm{H}\right), 6.93-6.95$ $(\mathrm{m}, 2 \mathrm{H}), 7.23-7.28(\mathrm{~m}, 1 \mathrm{H}), 7.37-7.52(\mathrm{~m}, 6 \mathrm{H}) ;{ }^{13} \mathrm{C} \mathrm{NMR}\left(\mathrm{CDCl}_{3}, 100 \mathrm{MHz}\right) \delta 55.4,66.4,111.4$ (s), 114.2, 123.9 (s), 125.0, 125.1, 125.4, 129.8, 131.4, 131.7 (s), 142.3 (s), 146.6, 159.7 (s); MS (EI, $70 \mathrm{eV}$ ) m/z (rel int) $296\left(\mathrm{M}^{+}, 39\right), 121$ (100). Anal. Calcd for $\mathrm{C}_{17} \mathrm{H}_{16} \mathrm{~N}_{2} \mathrm{O}_{3}$ (296.32): C, 68.91; H, 5.44; N, 9.45. Found: C, 68.80; H, 5.29; N, 9.37.

3-(4-Bromophenyl)-4,4-ethylenedioxy-8-methyl-3,4-dihydroquinazoline $22 \mathrm{~b}$ : eluent for column chromatography: hexanes/diethyl ether $(1: 1, \mathrm{v} / \mathrm{v})$; yield $98 \%$; $\mathrm{mp} 194^{\circ} \mathrm{C}$ (colorless prisms, diethyl ether); IR (nujol) 1627 (vs), 1596 (vs), 1574 (vs), 1331 (vs), 1307 (vs), 1283 (vs), 1250 (vs), 1197 (s), 1172 (s), 1136 (vs), 1122 (vs), 1098 (s), 1088 (s), 1041 (vs), 1011 (vs), 992 (vs), 951 (vs), 911 (s), 856 (s), 822 (vs), 816 (vs), 784 (vs), 761 (vs) cm ${ }^{-1} ;{ }^{1} \mathrm{H} \mathrm{NMR}\left(\mathrm{CDCl}_{3}, 400 \mathrm{MHz}\right) \delta 2.51$ (s, $3 \mathrm{H}$ ), 3.55-3.59 (m, 2 H), 4.02-4.05 (m, 2 H), 7.13 (t, 1 H, J = 7.7 Hz), 7.26 (d, 2 H, J = 7.7 Hz), 7.29$7.33(\mathrm{~m}, 2 \mathrm{H}), 7.37$ (m, $1 \mathrm{H}), 7.50-7.54(\mathrm{~m}, 2 \mathrm{H}) ;{ }^{13} \mathrm{C} \mathrm{NMR}\left(\mathrm{CDCl}_{3}, 100 \mathrm{MHz}\right) \delta 17.6,66.2,111.8$ (s), 122.2, 122.7 (s), 124.0 (s), 125.1, 130.8, 131.8, 132.3, 133.4 (s), 138.7 (s), 140.5 (s), 144.5; MS (EI, $70 \mathrm{eV}) \mathrm{m} / \mathrm{z}$ (rel int) $360\left(\mathrm{M}^{+}+2,58\right), 358\left(\mathrm{M}^{+}, 69\right), 144$ (100). Anal. Calcd for $\mathrm{C}_{17} \mathrm{H}_{15} \mathrm{BrN}_{2} \mathrm{O}_{2}$ (359.22): C, 56.84; H, 4.21; N, 7.80. Found: C, 56.76; H, 4.12; N, 7.64.

3-(4-Chlorophenyl)-4,4-ethylenedioxy-8-methyl-3,4-dihydroquinazoline 22c: eluent for column chromatography: hexanes/diethyl ether $(1: 1, \mathrm{v} / \mathrm{v})$; yield $97 \%$; $\mathrm{mp} 176^{\circ} \mathrm{C}$ (colorless prisms, diethyl ether); IR (nujol) 1627 (vs), 1596 (vs), 1576 (vs), 1331 (vs), 1305 (vs), 1287 (vs), 1250 (vs), 1197 (s), 1137 (vs), 1082 (vs), 1044 (vs), 1013 (vs), 989 (vs), 951 (vs), 822 (vs), 782 (s), 770 (s), 761 (vs) $\mathrm{cm}^{-1} ;{ }^{1} \mathrm{H}$ NMR $\left(\mathrm{CDCl}_{3}, 300 \mathrm{MHz}\right) \delta 2.51(\mathrm{~s}, 3 \mathrm{H}), 3.53-3.57(\mathrm{~m}, 2 \mathrm{H}), 4.01-4.06(\mathrm{~m}, 2 \mathrm{H}), 7.15(\mathrm{t}, 1$ $\mathrm{H}, J=7.6 \mathrm{~Hz}), 7.27-7.30(\mathrm{~m}, 2 \mathrm{H}), 7.37(\mathrm{~s}, 4 \mathrm{H}), 7.40(\mathrm{~s}, 1 \mathrm{H}) ;{ }^{13} \mathrm{C} \mathrm{NMR}\left(\mathrm{CDCl}_{3}, 75 \mathrm{MHz}\right) \delta 17.7$, 66.1, $111.4(\mathrm{~s}), 122.2,123.7$ (s), 125.0, 129.2, 130.7, 131.4, 133.1 (s), 134.5 (s), 137.7 (s), 140.3 (s), 
144.6; MS (EI, $70 \mathrm{eV}) \mathrm{m} / \mathrm{z}$ (rel int) $316\left(\mathrm{M}^{+}+2,25\right), 314\left(\mathrm{M}^{+}, 83\right), 125$ (100). Anal. Calcd for $\mathrm{C}_{17} \mathrm{H}_{15} \mathrm{ClN}_{2} \mathrm{O}_{2}$ (314.77): C, 64.87; H, 4.80; N, 8.90. Found: C, 64.79; H, 4.69; N, 8.73.

4,4-Ethylenedioxy-8-methyl-3-(4-methylphenyl)-3,4-dihydroquinazoline 22d: eluent for column chromatography: hexanes/diethyl ether $(1: 1, \mathrm{v} / \mathrm{v})$; yield $99 \%$; mp $110^{\circ} \mathrm{C}$ (colorless prisms, diethyl ether); IR (nujol) 1620 (vs), 1592 (vs), 1513 (vs), 1330 (vs), 1304 (vs), 1245 (vs), 1194 (vs), 1169 (vs), 1138 (vs), 1117 (vs), 1049 (vs), 1025 (vs), 975 (vs), 952 (vs), 908 (s), 856 (s), 823 (s), 764 (vs), $725(\mathrm{~s}) \mathrm{cm}^{-1} ;{ }^{1} \mathrm{H} \mathrm{NMR}\left(\mathrm{CDCl}_{3}, 300 \mathrm{MHz}\right) \delta 2.37(\mathrm{~s}, 3 \mathrm{H}), 2.52(\mathrm{~s}, 3 \mathrm{H}), 3.52-3.56(\mathrm{~m}, 2 \mathrm{H})$, 3.99-4.04 (m, 2 H), 7.13 (t, $1 \mathrm{H}, J=7.6 \mathrm{~Hz}), 7.18-7.21$ (m, $2 \mathrm{H}), 7.25-7.29$ (m, $2 \mathrm{H}), 7.31-7.34$ (m, $2 \mathrm{H}), 7.43(\mathrm{~s}, 1 \mathrm{H}) ;{ }^{13} \mathrm{C} \mathrm{NMR}\left(\mathrm{CDCl}_{3}, 75 \mathrm{MHz}\right) \delta 17.7,21.0,66.2,111.5(\mathrm{~s}), 122.4,123.7$ (s), 124.7, 129.6, 129.8, 130.6, 132.8 (s), 136.6 (s), 138.5 (s), 140.6 (s), 145.3; MS (EI, 70 eV) m/z (rel int) $294\left(\mathrm{M}^{+}, 74\right), 105$ (100). Anal. Calcd for $\mathrm{C}_{18} \mathrm{H}_{18} \mathrm{~N}_{2} \mathrm{O}_{2}$ (294.35): C, 73.45; H, 6.16; N, 9.52. Found: C, 73.32; H, 6.02; N, 9.35.

3-(4-Bromophenyl)-4,4-ethylenedioxy-6-methyl-3,4-dihydroquinazoline 22e: eluent for column chromatography: hexanes/diethyl ether $(1: 9, \mathrm{v} / \mathrm{v})$; yield $77 \%$; mp $136^{\circ} \mathrm{C}$ (colorless prisms, diethyl ether); IR (nujol) 1623 (vs), 1610 (vs), 1579 (vs), 1568 (vs), 1334 (vs), 1307 (vs), 1180 (vs), 1061 (vs), 1001 (vs), 982 (vs), 968 (vs), 951 (vs), 922 (s), 878 (s), 840 (s), 777 (vs), 755 (s), 727 (s) cm ${ }^{-1}$; ${ }^{1} \mathrm{H} \mathrm{NMR}\left(\mathrm{CDCl}_{3}, 400 \mathrm{MHz}\right) \delta 2.39$ (s, $\left.3 \mathrm{H}\right), 3.58-3.62$ (m, $\left.2 \mathrm{H}\right), 4.08-4.12(\mathrm{~m}, 2 \mathrm{H})$, 7.23-7.25 (m, $2 \mathrm{H})$, 7.27-7.30 (m, $2 \mathrm{H}), 7.32-7.35(\mathrm{~m}, 2 \mathrm{H}), 7.54-7.56(\mathrm{~m}, 2 \mathrm{H}) ;{ }^{13} \mathrm{C} \mathrm{NMR}\left(\mathrm{CDCl}_{3}, 100 \mathrm{MHz}\right) \delta$ 21.3, 66.3, 111.5 (s), 122.8 (s), 123.6 (s), 124.6, 125.2, 131.0, 131.9, 132.3, 135.4 (s), 138.4 (s), 139.7 (s), 145.0; MS (EI, $70 \mathrm{eV}) \mathrm{m} / \mathrm{z}$ (rel int) $360\left(\mathrm{M}^{+}+2,37\right), 358\left(\mathrm{M}^{+}, 40\right), 144$ (100). Anal. Calcd for $\mathrm{C}_{17} \mathrm{H}_{15} \mathrm{BrN}_{2} \mathrm{O}_{2}$ (359.22): C, 56.84; H, 4.21; N, 7.80. Found: C, 56.66; H, 4.07; N, 7.66.

3-(4-Chlorophenyl)-4,4-ethylenedioxy-6-methyl-3,4-dihydroquinazoline $22 \mathrm{f}$ : eluent for column chromatography: hexanes/diethyl ether $(1: 9, \mathrm{v} / \mathrm{v})$; yield $85 \%$; mp $110^{\circ} \mathrm{C}$ (colorless prisms, diethyl ether); IR (nujol) 1630 (vs), 1607 (vs), 1585 (s), 1567 (s), 1338 (vs), 1304 (s), 1282 (s), 1182 (s), 1001 (m), 1089 (s), 1065 (vs), 1004 (s), 980 (vs), 965 (s), 950 (vs), 830 (s), 780 (s), 734 (s) cm ${ }^{-1}$; ${ }^{1} \mathrm{H}$ NMR $\left(\mathrm{CDCl}_{3}, 400 \mathrm{MHz}\right) \delta 2.39$ (s, $\left.3 \mathrm{H}\right), 3.58-3.61$ (m, $\left.2 \mathrm{H}\right), 4.09-4.12$ (m, $\left.2 \mathrm{H}\right)$, 7.23-7.25 (m, 2 $\mathrm{H})$, 7.29-7.30 (m, $2 \mathrm{H}), 7.39-7.41(\mathrm{~m}, 4 \mathrm{H}) ;{ }^{13} \mathrm{C} \mathrm{NMR}\left(\mathrm{CDCl}_{3}, 100 \mathrm{MHz}\right) \delta 21.3,66.3,111.6(\mathrm{~s})$, 123.7 (s), 124.7, 125.2, 129.3, 131.1, 131.7, 134.7 (s), 135.5 (s), 137.9 (s), 139.9 (s), 145.1; MS (EI, $70 \mathrm{eV}) \mathrm{m} / \mathrm{z}$ (rel int) $316\left(\mathrm{M}^{+}+2,26\right), 314\left(\mathrm{M}^{+}, 99\right), 270$ (100). Anal. Calcd for $\mathrm{C}_{17} \mathrm{H}_{15} \mathrm{ClN}_{2} \mathrm{O}_{2}$ (314.77): C, 64.87; H, 4.80; N, 8.90. Found: C, 64.63; H, 4.70; N, 8.77. 


\section{Computational Details}

All calculations were carried out with the Gaussian $03^{6}$ suite of programs. An intensive characterization of the potential energy surface was done at the HF/6-31G*7 theoretical level and then with the $\mathrm{B}^{2} \mathrm{LYP}^{8}$ functional using the $6-31+\mathrm{G}^{* *}$ basis set. All the reported stationary points were fully optimized by analytical gradient techniques. Harmonic frequency calculations at each level of theory verified the identity of each stationary point as a minimum or a transition state, and were used to provide an estimation of the zero-point vibrational energies (ZPVE), which were not scaled. 7 Second order perturbation analyses were achieved with the NBO (natural bond orbital) method. $^{9}$

TABLE S1. Electronic energy including the zero point vibrational correction (in hartrees), relative energy (E. relat. in $\mathrm{kcal} \cdot \mathrm{mol}^{-1}$ ) and first frequency $\left(\mathrm{cm}^{-1}\right)$ of the stationary points found in the transformations $\mathbf{9} \rightarrow \mathbf{1 1}$, $12 \rightarrow 14$, and $15 \rightarrow 17$.

\begin{tabular}{cccc}
\hline \hline \multirow{2}{*}{ Method } & \multicolumn{3}{c}{ B3LYP/6-31+G** } \\
\cline { 2 - 4 } & Electronic E. & E. relat. & Freq \\
\hline TS1a & -630.759560 & 0 & 25.5 \\
10 & -630.713327 & 29.01 & -1171.0 \\
TS2a & -630.746001 & 8.51 & 68.8 \\
$\mathbf{1 1}$ & -630.715309 & 27.77 & -443.4 \\
$\mathbf{1 2}$ & -1276.715519 & -24.95 & 131.2 \\
TS1b & -1276.666011 & 0 & 23.9 \\
$\mathbf{1 3}$ & -1276.694508 & 13.18 & -975.4 \\
TS2b & -1276.662217 & 33.45 & 68.4 \\
$\mathbf{1 4}$ & -1276.747219 & -19.89 & -308.7 \\
$\mathbf{1 5}$ & -402.960834 & 0 & 47.8 \\
TS1c & -402.896770 & 40.20 & 21.2 \\
$\mathbf{1 6}$ & -402.925190 & 22.37 & -938.1 \\
TS2c & -402.899379 & 38.56 & 49.6 \\
$\mathbf{1 7}$ & -402.991855 & -19.47 & -310.3 \\
\hline & & & 39.2 \\
\hline
\end{tabular}


Selected Interaction from the Second Order Perturbation Analysis

Structure 9

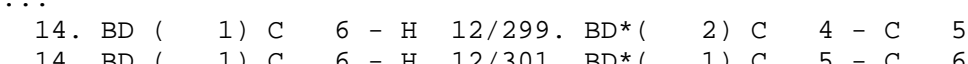

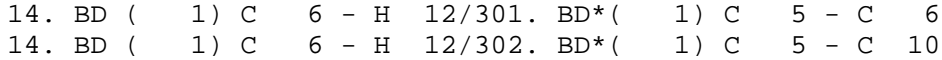

46. LP ( 1) $\mathrm{N} 3 \quad / 304 . \mathrm{BD}^{*}\left(\begin{array}{lllll}1 & \mathrm{C} & 6 & \mathrm{H} & \mathrm{H}\end{array}\right.$

$304 . \mathrm{BD}(\mathrm{1}, 1) \mathrm{C}$

47. LP ( 1) 011

/304. BD*( 1) C 6 - H 12

48. LP $\left(\begin{array}{llll}2 & 0 & 11\end{array}\right.$

/304. BD*( 1) C $6-$ H 12

50. LP ( 2) 013

/304. BD*( 1) C 6 - H 12

$\begin{array}{lll}0.68 & 0.55 & 0.019 \\ 0.60 & 0.94 & 0.021 \\ 3.61 & 1.09 & 0.056 \\ 0.78 & 0.77 & 0.023 \\ 1.07 & 1.02 & 0.030 \\ \mathbf{5 . 1 8} & 0.73 & 0.056 \\ \mathbf{6 . 2 0} & 0.73 & 0.060\end{array}$

Structure 12

14. BD (

1) $\mathrm{C} 6-\mathrm{H}$ 12/310. $\mathrm{BD}^{*}\left(\begin{array}{lllll}1 & \mathrm{C} & 5 & -\mathrm{C} & 10\end{array}\right.$

4.74

1.09

0.064

54. LP ( 1) N 3

/312. $B D^{*}($

1) $\mathrm{C} \quad 6-\mathrm{H} 12$

1.85

0.79

0.036

56. LP ( 2) S 11

/312. BD*(

1) $\mathrm{C} \quad 6-\mathrm{H} 12$

2.33

0.68

0.036

57. LP ( 1) S 13

/312. $B D^{*}($

1) $\mathrm{C} 6$ - H 12

0.65

1.10

0.024

58. LP ( 2) S 13

/312. BD*(

1) $\mathrm{C} \quad 6-\mathrm{H} 12$

2.65

0.69

0.039

Structure TS1a

47. LP ( 1) 011

48. LP ( 2) 011

50. LP (

2) 013

$\cdots$

Structure TS1b

54. LP ( 1) N 3

56. LP ( 2) S 11

58. LP ( 2) S 13
/304. BD* (

1) $\mathrm{C} \quad 6-\mathrm{H} 12$

/304. BD*(

1) $\mathrm{C} \quad 6-\mathrm{H} 12$

/304. BD* (

1) $\mathrm{C} \quad 6-\mathrm{H} 12$

/312. BD* (

1) $\mathrm{C} \quad 6-\mathrm{H} 12$

/312. BD*(

1) $\mathrm{C} \quad 6-\mathrm{H} 12$

/312. BD*(

1) $\mathrm{C} \quad 6-\mathrm{H} 12$
0.51

0.80

11.86

0.52

0.52

12.19

$0.51 \quad 0.51 \quad 0.015$

$5.28 \quad 0.43 \quad 0.045$

$\begin{array}{lll}4.95 & 0.44 & 0.043\end{array}$ 

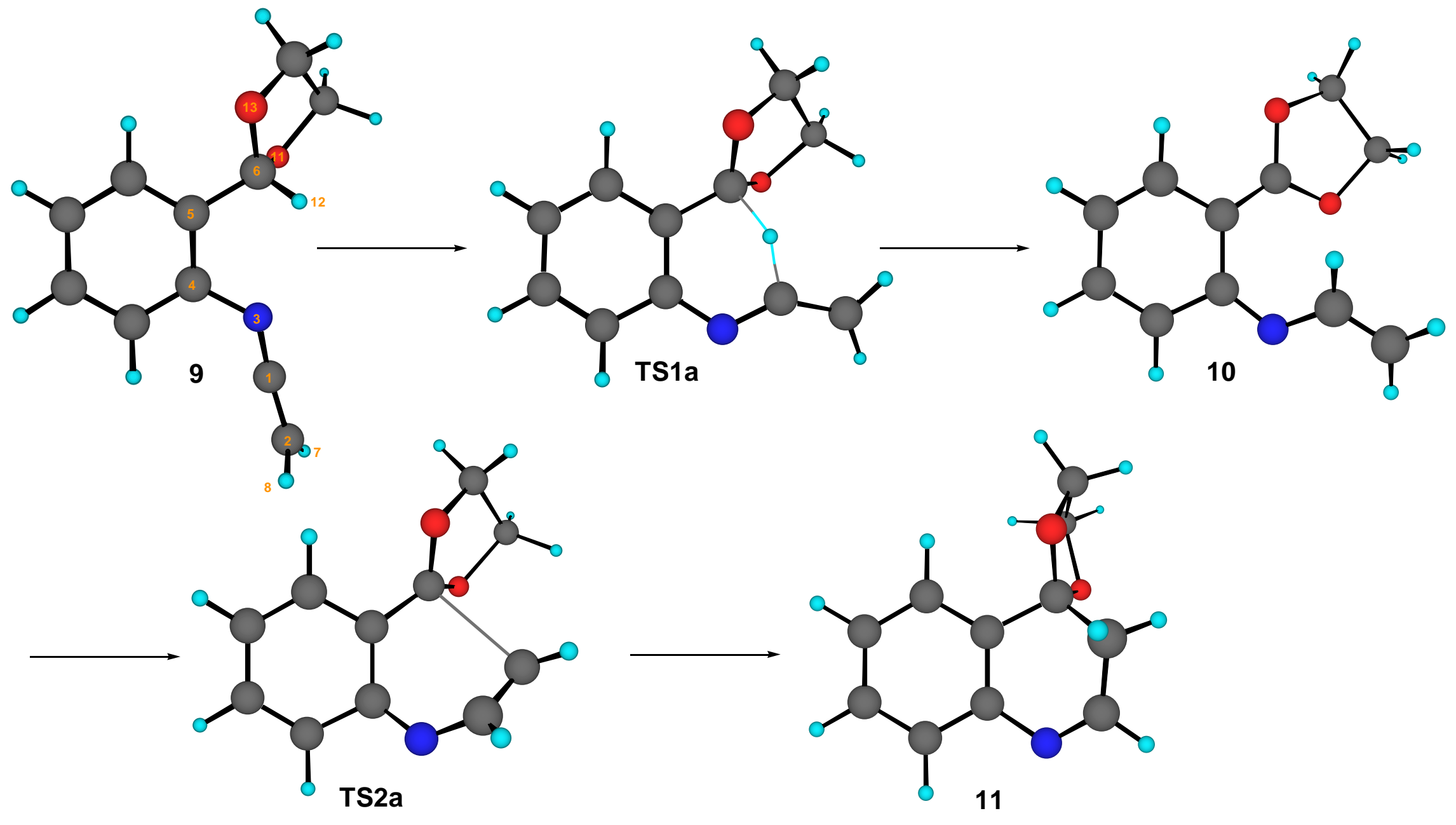

Figure S1. B3LYP/6-31+G**-optimized geometries of stationary points found in the transformation $\mathbf{9} \rightarrow \mathbf{1 1}$. 


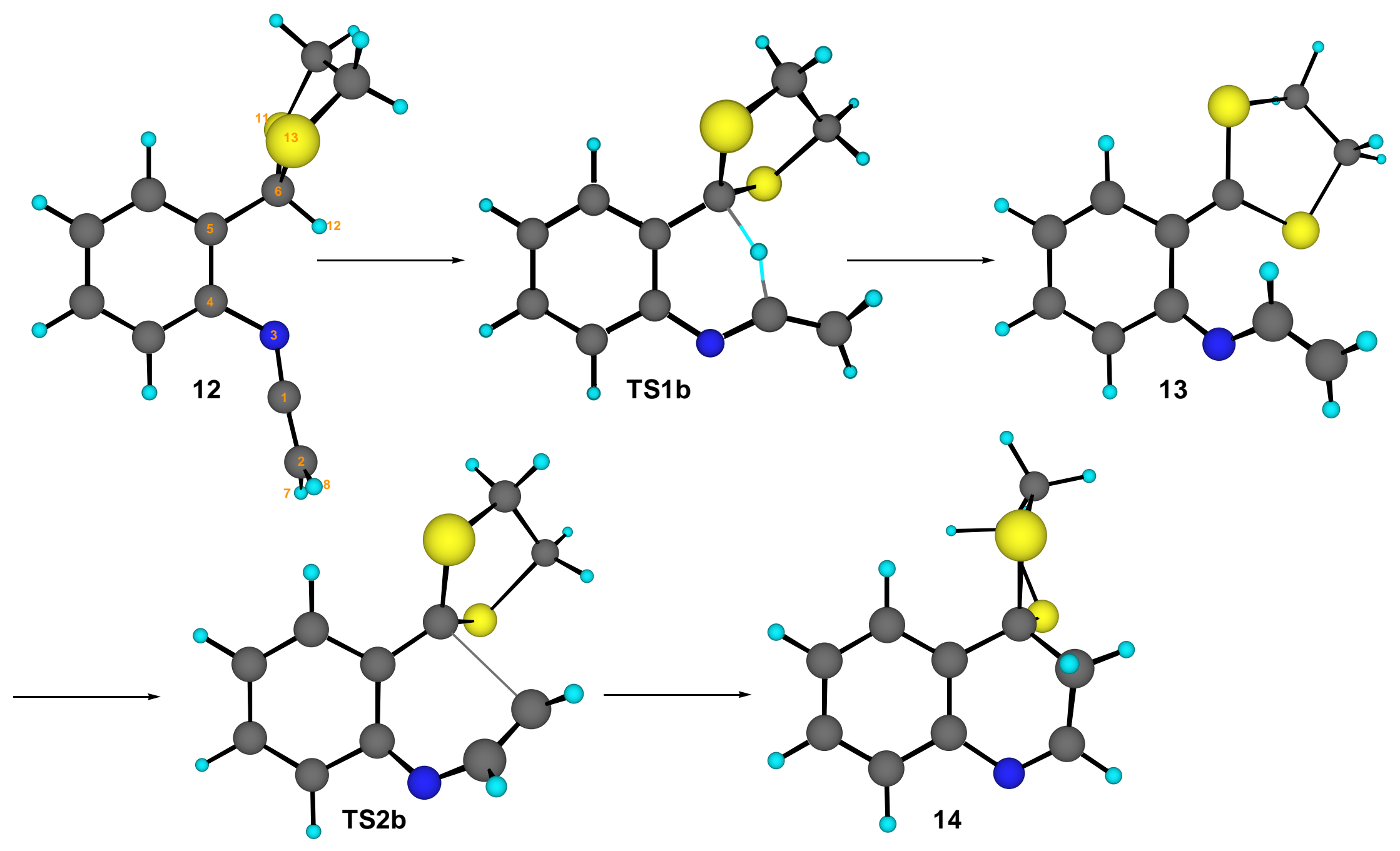

Figure S2. B3LYP/6-31+G**-optimized geometries of stationary points found in the transformation $\mathbf{1 2} \rightarrow \mathbf{1 4}$. 

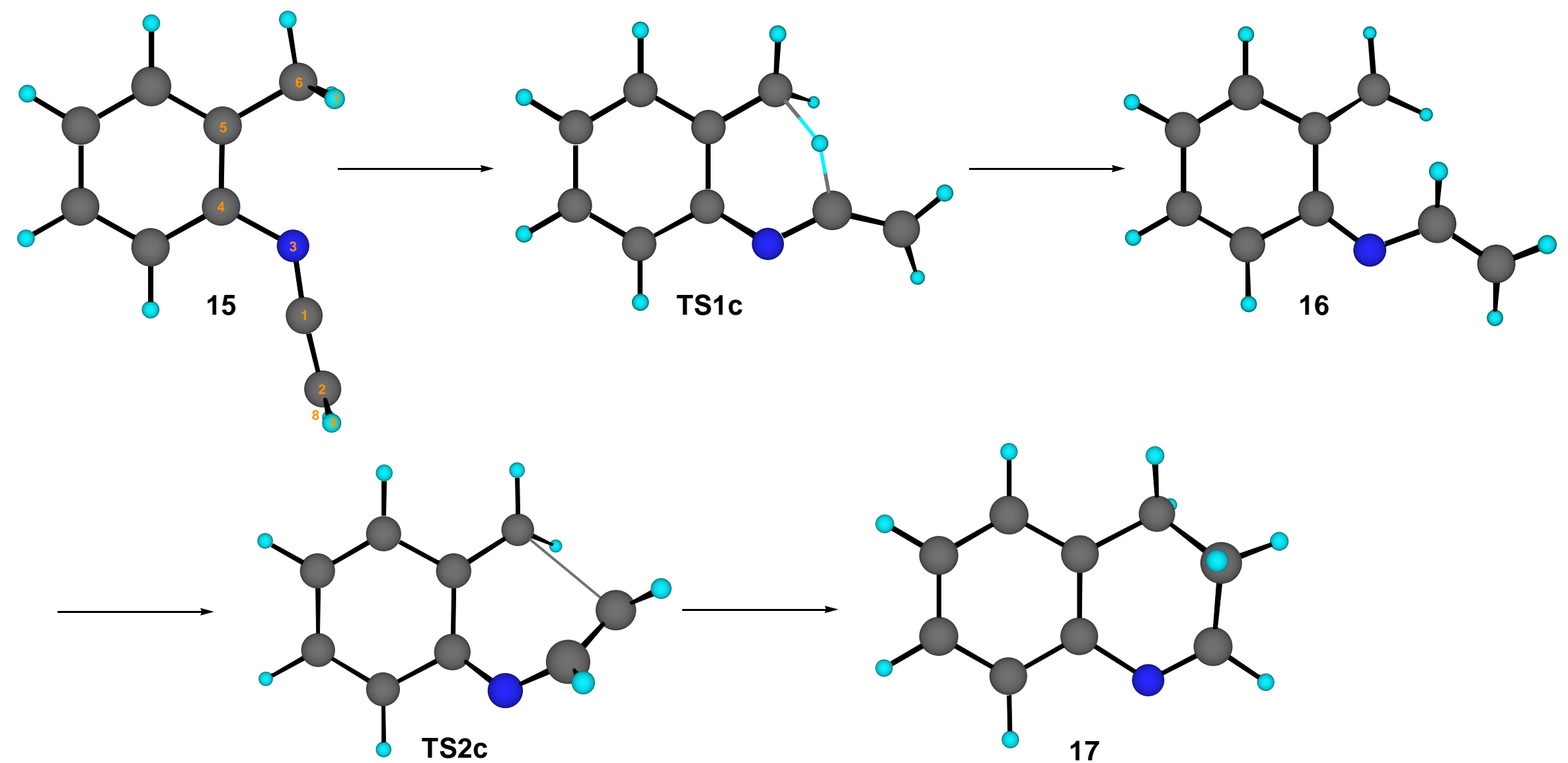

Figure S3. B3LYP/6-31+G**-optimized geometries of stationary points found in the transformation $\mathbf{1 5} \rightarrow \mathbf{1 7}$. 
Cartesian coordinates of the stationary points found in the transformations $9 \rightarrow \mathbf{1 1}, \mathbf{1 2} \rightarrow \mathbf{1 4}$, and $\mathbf{1 5} \rightarrow \mathbf{1 7}$ optimized at the B3LYP/6-31+G** theoretical level.

\begin{tabular}{|c|c|c|}
\hline 9 & TS1a & 10 \\
\hline C,2.8939819186,0.5702178524,-0.9974088779 & $\mathrm{C}, 0.7781613111,-1.5256314252,1.3273783815$ & C,1.7719532977,-0.5744588653,-1.5150329709 \\
\hline C,4.0164984259,1.078325639,-1.4587126808 & $\mathrm{C}, 1.7896570216,-1.7478842865,2.1828772349$ & C,3.0511034261,-0.4529444645,-1.9249318074 \\
\hline $\mathrm{N}, 1.8984762252,0.0826290706,-0.4718169446$ & $\mathrm{~N}, 0.1177841645,-2.1269970457,0.3822968133$ & N,0.7554360195,0.2331906096,-1.971438672 \\
\hline $\mathrm{C}, 0.6313132512,-0.1175062633,-1.0790282661$ & $C,-0.4979012636,-1.3994682063,-0.5930943841$ & $C,-0.4091959856,0.3072003604,-1.372681827$ \\
\hline$C,-0.4764987152,-0.3248451984,-0.2389023721$ & $C,-0.5558442923,0.0310795605,-0.5588716845$ & $C,-0.7713475455,-0.1353894218,-0.0042674676$ \\
\hline$C,-0.3191103606,-0.2700760415,1.2601634157$ & $\mathrm{C}, 0.2540455602,0.7481552592,0.4085584389$ & $\mathrm{C}, 0.1134023638,-0.1106174199,1.046906874$ \\
\hline $\mathrm{H}, 4.2323821864,2.1348725767,-1.3414429848$ & $\mathrm{H}, 1.9579742719,-1.1045412549,3.0372007374$ & H,3.8100455451,-1.1516917299,-1.5897334799 \\
\hline $\mathrm{H}, 4.7395341986,0.4476543572,-1.9650503205$ & Н,2.4619189789,-2.5849215087,2.0224132695 & H,3.3479157587,0.3482363951,-2.5943888554 \\
\hline$C, 0.4737615855,-0.1472244023,-2.4731177254$ & $C,-1.2685989445,-2.0713775364,-1.5785757021$ & $C,-1.5133369178,0.8342074854,-2.1567993935$ \\
\hline$C,-1.7324550649,-0.5466271477,-0.8133822899$ & $C,-1.4744454987,0.7185363705,-1.3802906899$ & C,-2.1640409959,-0.4242598575,0.2896504679 \\
\hline $\mathrm{O},-0.548130731,1.0509942954,1.7360294978$ & $\mathrm{O}, 1.585733407,0.9545661934,0.1402628236$ & $\mathrm{O}, 1.3357825217,0.4481851064,1.0218541707$ \\
\hline $\mathrm{H}, 0.688902202,-0.5924687989,1.5584946491$ & $\mathrm{H}, 0.3434497941,-0.3018138165,1.3788472031$ & H,1.509133973,-1.3955530962,-0.8370561207 \\
\hline$O,-1.2924673639,-1.0823083607,1.9147556987$ & $\mathrm{O},-0.2726700278,1.8671157477,1.006630644$ & $\mathrm{O},-0.1920761522,-0.5800358416,2.2813397722$ \\
\hline$C,-1.6249470107,-0.4457784951,3.1568365336$ & $\mathrm{C}, 0.8110866641,2.765190849,1.339258725$ & $\mathrm{C}, 0.8209470761,-0.1150011904,3.2001656774$ \\
\hline $\mathrm{H},-1.3333154774,-1.0855798862,3.9976189507$ & $\mathrm{H}, 0.6776346973,3.1055513024,2.3679513992$ & $\mathrm{H}, 0.9947062186,-0.8997062814,3.9370069827$ \\
\hline $\mathrm{H},-2.7087129759,-0.2888481199,3.1838011647$ & $\mathrm{H}, 0.7718776016,3.6180694581,0.6541034437$ & $\mathrm{H}, 0.4599011896,0.7943287618,3.6924248459$ \\
\hline C,-0.8355259281,0.8798801817,3.1220272712 & C,2.0509483063,1.8928463035,1.1265996406 & $\mathrm{C}, 2.0005508913,0.1485191353,2.2673487111$ \\
\hline H,0.0972160639,0.8077917416,3.7002990654 & H,2.3466205243,1.3538392314,2.0338750477 & $\mathrm{H}, 2.6355632205,-0.7322174774,2.124906764$ \\
\hline $\mathrm{H},-1.4066549637,1.7458606212,3.4627146611$ & H,2.9080588178,2.4208562527,0.7082073559 & H,2.6091047731,1.011149581,2.5388736388 \\
\hline $\mathrm{C},-0.7820522417,-0.374432533,-3.0294997181$ & $\mathrm{C},-2.0893134676,-1.3669769123,-2.4368827105$ & $C,-2.8112058109,0.6339054129,-1.7954070485$ \\
\hline $\mathrm{H}, 1.3415164723,0.0045865915,-3.1082035281$ & $\mathrm{H},-1.2108527685,-3.1543099778,-1.6120534389$ & $\mathrm{H},-1.2488094623,1.2997662691,-3.1007633309$ \\
\hline$C,-1.8901833387,-0.5738002609,-2.1984240061$ & $C,-2.2138547503,0.0366808342,-2.3310213561$ & $C,-3.1470157112,-0.05889526,-0.5770339811$ \\
\hline $\mathrm{H},-0.8958603664,-0.4000402156,-4.109424248$ & $\mathrm{H},-2.6670284561,-1.9015967581,-3.1857687082$ & $\mathrm{H},-3.6114333655,0.9759108586,-2.4462947018$ \\
\hline$H,-2.870080745,-0.7554685811,-2.6295693323$ & $\mathrm{H},-2.8719507806,0.5795836906,-3.002122175$ & $\mathrm{H},-4.1877074446,-0.2553061518,-0.3387564924$ \\
\hline $\mathrm{H},-2.5781705398,-0.7150512437,-0.1561087416$ & $\mathrm{H},-1.5503427442,1.7979023115,-1.2894949307$ & $\mathrm{H},-2.4070080259,-0.8860410009,1.2407965053$ \\
\hline
\end{tabular}




\section{TS2a}

C, $2.5764075731,-0.514152395,0.2075944347$ C, $2.0706354576,-0.2148118784,1.4560284223$ N,2.206675035,-0.0320878025,-0.984191624 C, $0.9297983856,0.2137034869,-1.332886191$ C, $-0.2563671368,-0.1306365719,-0.5678796905$ C, $-0.3828863813,-0.0639507231,0.8482486932$ H, 2.4567888814,-0.7606373532,2.3143618532 $\mathrm{H}, 1.6773257142,0.7705545709,1.6454524553$ C,0.713287776,0.5473355203,-2.7136945469 C, $-1.4332141599,-0.5259193371,-1.277655794$ $\mathrm{O},-0.3928406402,1.1314184258,1.5025232663$ H,3.3841741513,-1.2507113347,0.1437651241 O,-1.1593507085,-0.9613178855,1.5015014018 C,-1.655523815,-0.3618916212,2.7195755063 H,-1.5568724897,-1.091569186,3.5247111658 $\mathrm{H},-2.7085063126,-0.1000428431,2.5736727635$ C,-0.75198924,0.8620942069,2.8694458256 $\mathrm{H}, 0.1546776243,0.6406605872,3.444698024$ H, $-1.2485514275,1.7481087783,3.2646889099$ C, $-0.484545046,0.3040394179,-3.3379435515$ H, $1.5773764454,0.8985147718,-3.2686704664$ C, $-1.5597159315,-0.2955746003,-2.6284899893$ $\mathrm{H},-0.5972682448,0.5292141978,-4.3950951552$ $\mathrm{H},-2.4920233066,-0.525548734,-3.1347676567$ H,-2.2716403817,-0.91614619,-0.7097317068

\section{1}

C,2.5257376209,-0.0224816485,0.330975531 C, $1.5578845064,-0.8193434979,1.1711969688$ $\mathrm{N}, 2.2476557969,0.5130851011,-0.7986375854$ C, $0.9343701884,0.354518775,-1.3023835909$ C, $-0.1405808275,-0.1194482944,-0.5176795002$ C,0.1194816341,-0.3584089248,0.9618293899 $\mathrm{H}, 1.6234576048,-1.8771739072,0.8764292853$ $\mathrm{H}, 1.8131258925,-0.7569098157,2.2330849861$ C,0.7171945199,0.6817847983,-2.6469622142 C,-1.3973172466,-0.2856417678,-1.1011984851 $\mathrm{O},-0.064461127,0.8571429666,1.7058949996$ $\mathrm{H}, 3.5486382025,0.0863763947,0.6960933222$ $\mathrm{O},-0.8122719508,-1.2977377916,1.504402387$ C,-1.5466487414,-0.6683455801,2.5627847134 H,-1.1100094962,-0.9312997869,3.5351337145 H,-2.5813790829,-1.0202433549,2.5214084718 C,-1.3810383084,0.8170093252,2.2541544186 $\mathrm{H},-1.400368325,1.4632731642,3.1344230829$ $\mathrm{H},-2.1224886477,1.1694452047,1.523813218$ C,-0.5450234863,0.5199525138,-3.2188556539 H, $1.5572011329,1.0526703268,-3.2256314902$ C,-1.6030023363,0.0299112818,-2.4481036518 $\mathrm{H},-0.7016788274,0.7697418272,-4.2642153306$ $\mathrm{H},-2.5839299221,-0.1108428587,-2.8926299685$ $\mathrm{H},-2.2086396268,-0.6889161866,-0.5043728395$
12

C,0.1871133455,-3.1536192582,1.516231249 C,0.2155469432,-4.3098146022,2.1431893002 $\mathrm{N}, 0.234415263,-2.0392208251,1.0041785068$ C,-0.4968708621,-1.5900145924,-0.1270199759 C, $-0.3769915672,-0.2352531394,-0.4961087595$ C, $0.4926311055,0.7042542003,0.3058276795$ $\mathrm{H}, 0.9107933979,-5.0850178475,1.8385544392$ H,-0.4556190579,-4.503770775,2.9732160105 C, $-1.3118955333,-2.45863248,-0.8723127133$ C, $-1.0918252721,0.2161774919,-1.6162493272$ $\mathrm{S}, 1.8332064438,1.517992303,-0.7222383887$ H,0.9950823317,0.1388993938,1.0880620862 S, $-0.5104500345,2.0332218492,1.1023734807$ C,0.9121686378,3.168056235,1.2767222802 H, 1.5848433303,2.8101031374,2.0620446704 H,0.520517217,4.147579562,1.5688885461 C, $1.623823738,3.2311090709,-0.0738635679$ H,2.6209909642,3.670989775,0.0261132515 H, $1.0445926309,3.8213215573,-0.7881835123$ C,-2.01146384,-1.9893967951,-1.9779071045 H,-1.3860867967,-3.4998054451,-0.5721382848 C,-1.9023302062,-0.6435347793,-2.3513558835 H,-2.6384228048,-2.6679430917,-2.548967596 $\mathrm{H},-2.4447344181,-0.2703755174,-3.2148044826$ H,-0.9964051187,1.2571504827,-1.9071152096 


\section{TS1b}

C, $1.5016755135,-1.5986218218,0.6387140718$ C. $2.786398987,-1.598056045,1.0396244726$ $\mathrm{N}, 0.7070528811,-2.3305178576,-0.09372565$ C, $-0.3899500038,-1.7759544243,-0.682398168$ C, $-0.8202464368,-0.4266050519,-0.415025705$ C, $0.0696331705,0.4915283789,0.2864310439$ H,3.1137069406,-0.9801262986,1.8668428846 $\mathrm{H}, 3.5301207935,-2.1813046062,0.5050889181$ C, $-1.2506784817,-2.6221199533,-1.4327260965$ C, $-2.1366971874,-0.0515923838,-0.7718603418$ S, $1.3471929163,1.3175429186,-0.6608446268$ H,0.8763273522,-0.5478752294,0.948927977 S,-0.617554291,1.5574220614,1.566144054 C, $0.5766863896,2.9442750943,1.3642064226$ $\mathrm{H}, 0.7712437771,3.3631499381,2.3555658336$ $\mathrm{H}, 0.12234325,3.710718355,0.7311226993$ C, $1.8528835626,2.3949730641,0.7288851945$ $\mathrm{H}, 2.4443223335,1.8163388193,1.4444049984$ $\mathrm{H}, 2.4737005774,3.197346069,0.3202140631$ C,-2.4913910894,-2.1877898826,-1.8506133461 H,-0.9033778617,-3.6286890764,-1.6407691111 C,-2.9510783427,-0.8987144331,-1.5027152075 $\mathrm{H},-3.1287928462,-2.8505118205,-2.4289771317$ $\mathrm{H},-3.9314637023,-0.5619085404,-1.8250841562$ H,-2.4751352737,0.9491124639,-0.5211823047
C,2.5217534384,-0.6187968297,-0.1514621177 C $3.7510345096,-0.4881808769,0.3906940975$ $\mathrm{N}, 2.0869807577,0.1818795507,-1.1816025179$ C, $0.8341821976,0.2171226976,-1.5766862581$ C, $-0.3733124596,-0.2447182469,-0.8410403515$ C, $-0.5314249758,-0.1036205125,0.530218271$ $\mathrm{H}, 4.0876133332,-1.1624976528,1.1703886973$ $\mathrm{H}, 4.4207791361,0.3011820531,0.064400635$ C,0.611160149,0.694566629,-2.9304088465 C,-1.4955589143,-0.6836450428,-1.6562100247 $\mathrm{S}, 0.5112473969,0.8882336871,1.5578611408$ $\mathrm{H}, 1.8632333618,-1.4189977798,0.2006948683$ S,-1.9144063066,-0.8174652877,1.3909501043 C,-1.8308140944,0.3117750105,2.8299843031 $\mathrm{H},-2.347987346,-0.1758248866,3.6610505489$ $\mathrm{H},-2.3397373167,1.2481346871,2.5853532323$ C, $-0.3582032067,0.5397671992,3.136512263$ $\mathrm{H}, 0.0914779806,-0.340718759,3.6038783769$ H, $-0.2076943965,1.4071854145,3.78547179$ C,-0.5229617865,0.3798382072,-3.6140747942 H,1.446682665,1.1995986276,-3.404145973 C,-1.5683220225,-0.3819360098,-2.9820109198 $\mathrm{H},-0.6315271179,0.671865288,-4.6548443309$ H,-2.4397194772,-0.6786934424,-3.5577821646 H,-2.3266405794,-1.1897181435,-1.1773217085
TS2b

C,2.6808375765,-0.7724160246,-0.2108347003 C, $2.2627624675,-0.4938762062,1.0739857264$ $\mathrm{N}, 2.2959470632,-0.2199054441,-1.3653800738$ C, $1.0335125682,0.1364653652,-1.6701337066$ C, $-0.1533212375,-0.1671564559,-0.8763617356$ C, $-0.2165974589,-0.1023746807,0.5557513754$ $\mathrm{H}, 2.6061890491,-1.1391672482,1.8800273256$ $\mathrm{H}, 1.9521736785,0.4985967872,1.3466017546$ C, $0.8199045786,0.5347927432,-3.0333010807$ C, $-1.3523488559,-0.4746562716,-1.591955531$ S, $-0.2021371932,1.5191537005,1.3043852303$ H,3.4267785075,-1.5630823279,-0.3419475808 S, $-1.2048314651,-1.265863789,1.4787106095$ C,-1.6023672451,-0.1566360987,2.8912879909 $\mathrm{H},-1.657378051,-0.7743665418,3.792106511$ $\mathrm{H},-2.5733199517,0.3139885766,2.7166907587$ C, $-0.4953213108,0.8921295785,2.9955361065$ $\mathrm{H}, 0.4278624928,0.4617306809,3.3959716199$ $\mathrm{H},-0.8000153703,1.7370790991,3.6193132576$ C,- $-0.3996018997,0.3854451277,-3.6442953392$ $\mathrm{H}, 1.694873411,0.8587689521,-3.5876687041$ C,-1.4873779463,-0.1832956639,-2.9318508311 $\mathrm{H},-0.5215225615,0.659723726,-4.6884820176$ $\mathrm{H},-2.4438262834,-0.3357243186,-3.4226115358$ $\mathrm{H},-2.2124332503,-0.8213763397,-1.028843956$ 


\section{4}

C,2.6764594455,-0.0547267069,-0.0567563091 C, $1.7254202819,-0.8914627072,0.7636340608$ $\mathrm{N}, 2.3688359236,0.5131776794,-1.1625500545$ C, $1.045649676,0.3603323797,-1.6365804365$ C, $-0.0229118634,-0.1009241181,-0.8257824307$ C, $0.2801581339,-0.381501564,0.6415370368$ H, 1.7707594688,-1.9238051343,0.3822026707 H,2.0306297425,-0.9261606054,1.8122368852 C, $0.8098481276,0.7116938037,-2.9716913106$ C, $-1.2957425418,-0.2058176787,-1.3949317181$ $\mathrm{S}, 0.1327205486,1.2008059085,1.6437498757$ H,3.7052304092,0.049850584,0.2930424622 S, $-0.8831978033,-1.6268719856,1.4142301005$ C,-1.7000929983,-0.5221988735,2.6392638923 H,-1.2096388099,-0.6263927569,3.6100552882 H,-2.7424269931,-0.8460243861,2.7258817235 C,-1.6012904659,0.9136648801,2.1354851301 H,-1.8382781594,1.6340078735,2.9248344735 H, $-2.2711365414,1.0896736985,1.2884582993$ C, $-0.4654074763,0.5980488758,-3.5233131059$ H, 1.6504726142,1.0700298126,-3.5573056999 C, $-1.5195368449,0.1369983336,-2.7315906173$ $\mathrm{H},-0.6349477875,0.8666129738,-4.5617898256$ $\mathrm{H},-2.5167456641,0.0380984188,-3.1504442579$ H,-2.1134545161,-0.5857167492,-0.792646408
C,-1.5589504496,0.0205838818,-1.958554679 $\mathrm{C},-2.7302328436,0.0326723305,-2.5591713493$ $\mathrm{N},-0.4216812488,0.0072767143,-1.4981530958$ C, $-0.0543803191,0.0014945376,-0.1253131403$ C,1.3238680554,-0.0163752231,0.1684356636 C, $2.3553706071,-0.0288522505,-0.9324272785$ H, 2.2371725768,-0.9049149741,-1.5785838434 $\mathrm{H},-3.2122860819,0.9717522438,-2.8097680138$ $\mathrm{H},-3.230380857,-0.8964888456,-2.8113294509$ C,-1.0086030684,0.0130692923,0.9023950084 C, $1.7037059336,-0.0222426969,1.5177776351$ $\mathrm{H}, 3.3656840903,-0.0414728381,-0.514422093$ $\mathrm{H}, 2.2588258029,0.8501693168,-1.5781473035$ C,-0.605398532,0.0069474606,2.235694771 $\mathrm{H},-2.0641355478,0.0268673013,0.645447184$ C,0.7579726708,-0.0108193317,2.5448977212 $\mathrm{H},-1.3492745615,0.0160032537,3.0269411483$ H,1.0837229599,-0.015703906,3.5809819175 $\mathrm{H}, 2.7623280346,-0.036016555,1.7635460123$
TS1c

C,1.481892688,1.3604410023,-1.0126322595 C, $2.2042674954,2.4282190691,-1.3875873569$ $\mathrm{N}, 0.313388186,0.8388931005,-1.3145445815$ C, $-0.3933506954,0.1204175038,-0.4186168773$ C,0.1631766829,-0.2240565885,0.8727985149 C, $1.3245190697,0.4559702407,1.3287848114$ H, 1.9503365742,0.7176881321,-0.0192150822 H,3.2498263414,2.5164471416,-1.1186258546 $\mathrm{H}, 1.7367732227,3.2505302804,-1.921004047$ C,-1.5958124502,-0.5229096144,-0.8437580379 C, $-0.3810093555,-1.3503765924,1.5563106542$ H, $1.9692373912,-0.0582138874,2.0428377196$ H, $1.3036085738,1.5342987288,1.4545883082$ C,-2.1664919031,-1.5118255343,-0.079873252 H,-2.0178926678,-0.2250515676,-1.7978680529 C,-1.5381514403,-1.9534678879,1.1171627885 $\mathrm{H},-3.083053541,-1.991523523,-0.4107966594$ H,-1.9810480486,-2.7618073084,1.691080021 $\mathrm{H}, 0.0842543036,-1.6690892899,2.485281805$ 


\section{6}

C, $-0.2392020175,-0.3518026238,-2.3517963845$ C, $-0.6462940932,-0.0409296076,-3.5982163437$

N,-0.960422796,0.0157469294,-1.2331452378

C, $-0.4616630553,0.097314828,-0.0298797464$

C, $0.9820315294,0.0683969286,0.372099781$

C, $1.9983002412,0.3982926282,-0.4654076069$

$\mathrm{H}, 0.6613610151,-0.9579963887,-2.213840684$

H, $-0.0833255191,-0.3724930719,-4.4636496963$

$\mathrm{H},-1.5309394547,0.5667637905,-3.7592996008$

C,-1.4302716719,0.2112488823,1.053808669

C, $1.2755672762,-0.2190496693,1.7753432862$

H,3.0239425052,0.3866341645,-0.106623234

$\mathrm{H}, 1.8386961097,0.7377119294,-1.4801223808$

C,-1.0742408259,0.0387744169,2.3521289969

H,-2.4631920988,0.3590099646,0.7565512395

C, $0.3024631856,-0.2235969638,2.7180484781$

H,-1.8291804478,0.0712695966,3.1324679949

H,0.5492289498,-0.4013422512,3.7605105915

$\mathrm{H}, 2.3162251017,-0.371679157,2.0492476561$

\section{TS2c}

C,-0.2429110552,0.4459906946,2.3791148086 C,0.9215084012,-0.1955931928,2.7713775164 $\mathrm{N},-1.0648910952,0.1839443932,1.3594716109$ C, $-0.6583155044,-0.1667728663,0.1274045$ C,0.7367993699,-0.1610272965,-0.2976784607 C, $1.8024662448,-0.5793712738,0.5047377941$ H,-0.5530672667,1.3151554571,2.9671480838 $\mathrm{H}, 1.5104976815,0.2525982774,3.5691135332$ $\mathrm{H}, 1.0102860001,-1.2663892502,2.6845561339$ C,-1.6640347631,-0.1900846025,-0.8999888994 C, $1.0066081685,0.3042802,-1.6314431524$ $\mathrm{H}, 2.809921962,-0.2629910303,0.2371629693$ $\mathrm{H}, 1.7529764086,-1.5128117782,1.034834037$ C,-1.3362944152,0.0566192036,-2.2073785906 $\mathrm{H},-2.6962582533,-0.3057355032,-0.5848863919$ C,0.0086932204,0.3708407608,-2.5700814992 H,-2.1124543591,0.0755424004,-2.9672498019 $\mathrm{H}, 0.2431384815,0.6331549362,-3.5973418047$ $\mathrm{H}, 2.0420790103,0.4745759753,-1.9160221352$
17

C,-0.1396091784,-0.0894424287,2.4093380107 C, $1.2913061179,0.2977079176,2.1079724638$ N,-1.0472085087,-0.2681550721,1.5224680259 C, $-0.6920958346,-0.0996841576,0.1594905547$ C, $0.6508585926,-0.096455597,-0.2752624841$ C, $1.7301016658,-0.300183438,0.7645672424$ H,-0.4385923322,-0.205907698,3.4534657442 H, 1.347184981,1.3976112427,2.0727723604 $\mathrm{H}, 1.948455543,-0.02219165,2.9231825664$ C,-1.7300684206,0.0418320449,-0.7703459985 C, $0.9216455548,0.0504173235,-1.6370779293$ H,2.6776259621,0.1365531652,0.432713336 $\mathrm{H}, 1.9078036875,-1.3790409843,0.8920943459$ C,-1.4445825869,0.1997451221,-2.1271356824 $\mathrm{H},-2.7525642025,0.0271452739,-0.405983344$ C, $-0.1163872683,0.2020471284,-2.5628666594$ H,-2.2541197294,0.3153104508,-2.8418337987 $\mathrm{H}, 0.1129131837,0.3207177163,-3.6178843453$ H,1.9547406136,0.0509844969,-1.9778801535 


\section{References}

1 Ardakani, M. A.; Smalley, R. K.; Smith, R. M. J. Chem. Soc., Perkin Trans.1 1983, 2501.

2 Cuevas, J. C.; Mendoza, J.; Prados, P. J. Org. Chem. 1988, 53, 2055.

3 2-Azido-5-chlorobenzaldehyde $\mathbf{5 c}$ was prepared following the experimental procedure described by Mendoza for the preparation of compounds $\mathbf{5 b}$ and 5d, see reference 2 .

4 Taylor, E. C.; McKillop, A.; Hawks, G. H. Org. Synth. 1973, 52, 36.

5 Pracejus, H.; Wallura, G. J. Prakt. Chem. 1962, 19, 33.

6 Frisch, M. J.; Trucks, G. W.; Schlegel, H. B.; Scuseria, G. E.; Robb, M. A.; Cheeseman, J. R.; Montgomery, J. A.; Vreven, T.; Kudin, K. N.; Burant, J. C.; Millam, J. M.; Iyengar, S. S.; Tomasi, J.; Barone, V.; Mennucci, B.; Cossi, M.; Scalmani, G.; Rega, N.; Petersson, G. A.; Nakatsuji, H.; Hada, M.; Ehara, M.; Toyota, K.; Fukuda, R.; Hasegawa, J.; Ishida, M.; Nakajima, T.; Honda, Y.; Kitao, O.; Nakai, H.; Klene, M.; Li, X.; Knox, J. E.; Hratchian, H. P.; Cross, J. B.; Adamo, C.; Jaramillo, J.; Gomperts, R.; Stratmann, R. E.; Yazyev, O.; Austin, A. J.; Cammi, R.; Pomelli, C.; Ochterski, J. W.; Ayala, P. Y.; Morokuma, K.; Voth, G. A.; Salvador, P.; Dannenberg, J. J.; Zakrzewski, V. G.; Dapprich, S.; Daniels, A. D.; Strain, M. C.; Farkas, O.; Malick, D. K.; Rabuck, A. D.; Raghavachari, K.; Foresman, J. B.; Ortiz, J. V.; Cui, Q.; Baboul, A. G.; Clifford, S.; Cioslowski, J.; Stefanov, B. B.; Liu, G.; Liashenko, A.: Piskorz, P.; Komaromi, L.; Martin, R. L.; Fox, D. J.; Keith, T.; Al-Laham, M. A.; Peng, C. Y.; Nanayakkara, A.; Challacombe, M. P.; Gill, M. W.; Johnson, B. G.; Chen, W.; Wong, M. W.; Gonzalez, C.; Pople, J. A. Gaussian 03, Revision B.03; Gaussian, Inc.: Pittsburgh, PA, 2003.

7 Hehre, W. J.; Radom, L.; Schleyer, P. v. R.; Pople, J. A. in Ab Initio Molecular Orbital Theory, Wiley, New York, 1986, pp 71-82, and references cited therein.

8 a) Parr, R. G.; Yang, W. Density-Functional Theory of Atoms and Molecules, Oxford University Press, New York, 1989. b) Bartolotti, L. J.; Fluchichk, K. in Reviews in Computational Chemistry, Lipkowitz, K. B.; Boyds, D. B. Eds. VCH Publishers, New York, 1996, Vol. 7; pp 187-216. c) Kohn, W.; Becke, A. D.; Parr, R. G. J. Phys. Chem. 1996, 100, 12974-12980. d) Ziegler, T. Chem. Rev. 1991, 91, 651-667.

9 (a) Redd, A. E.; Weinstock, R. B.; Weinhold, F. J. Chem. Phys. 1985, 83, 735-746. (b) Reed, A. E.; Curtiss, L. A.; Weinhold, F. Chem. Rev. 1988, 88, 899-926. (c) Reed, A. E.; Schleyer, P. v. R. J. Am. Chem. Soc. 1990, 112, 1434-1445. 


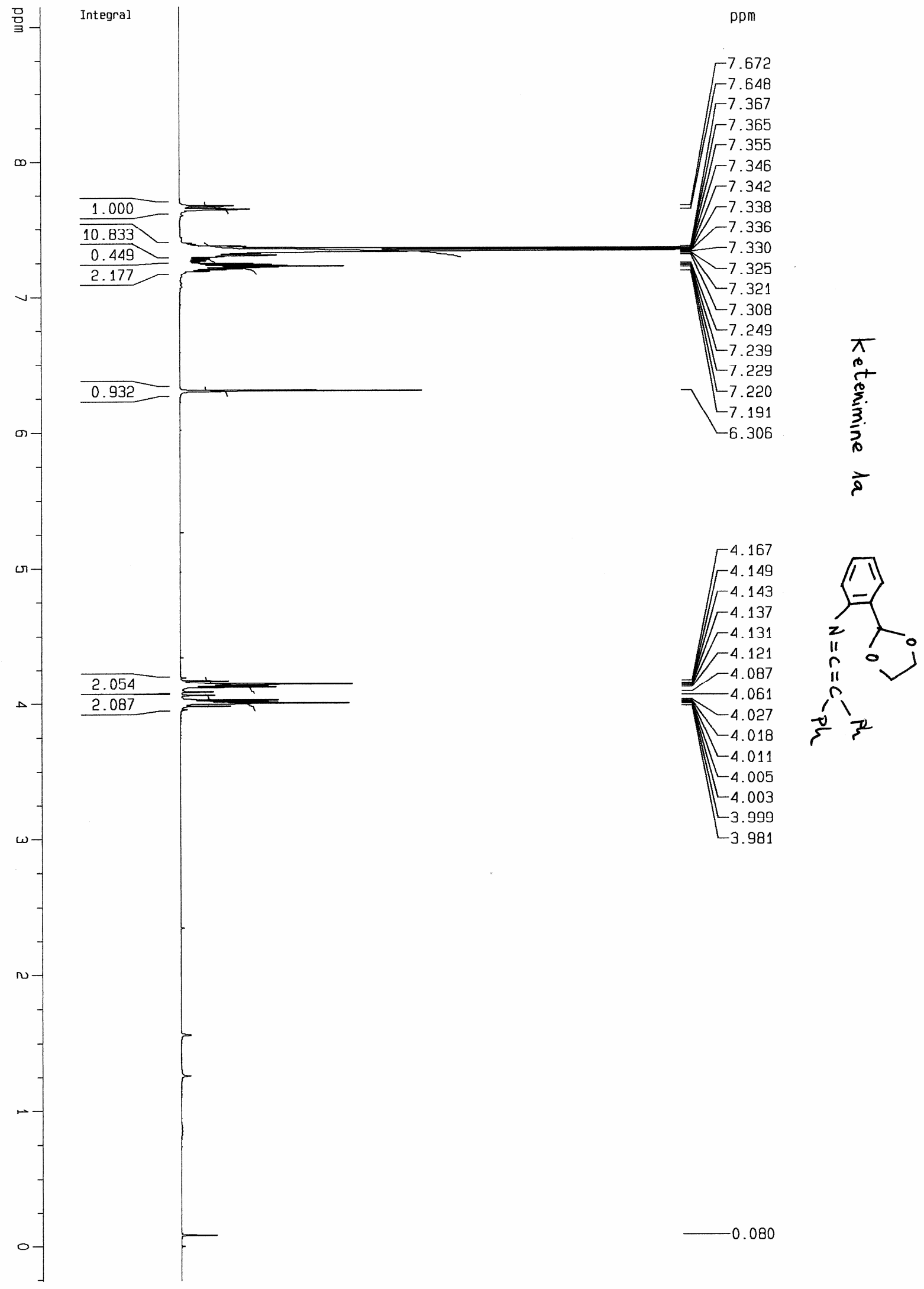




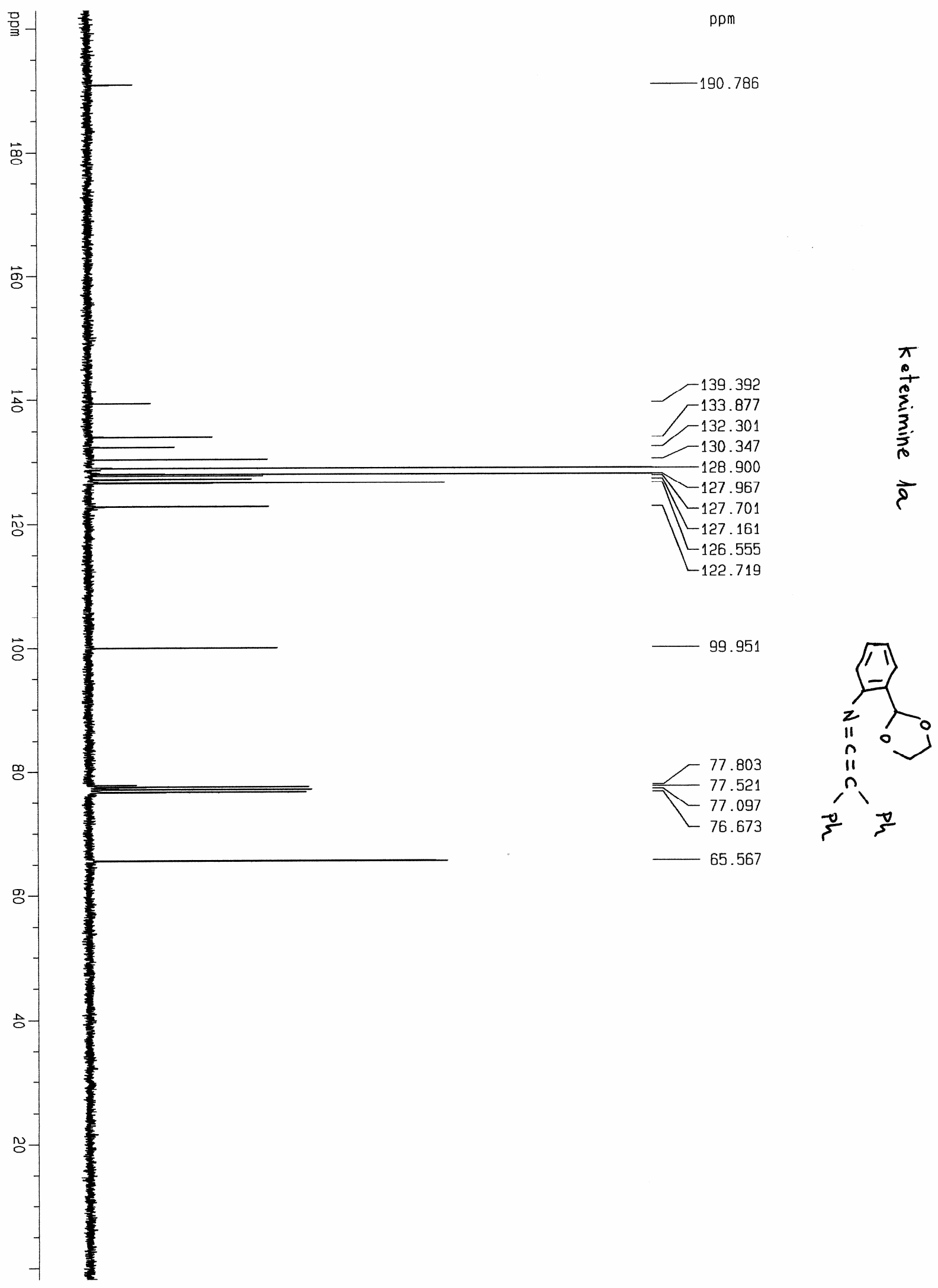




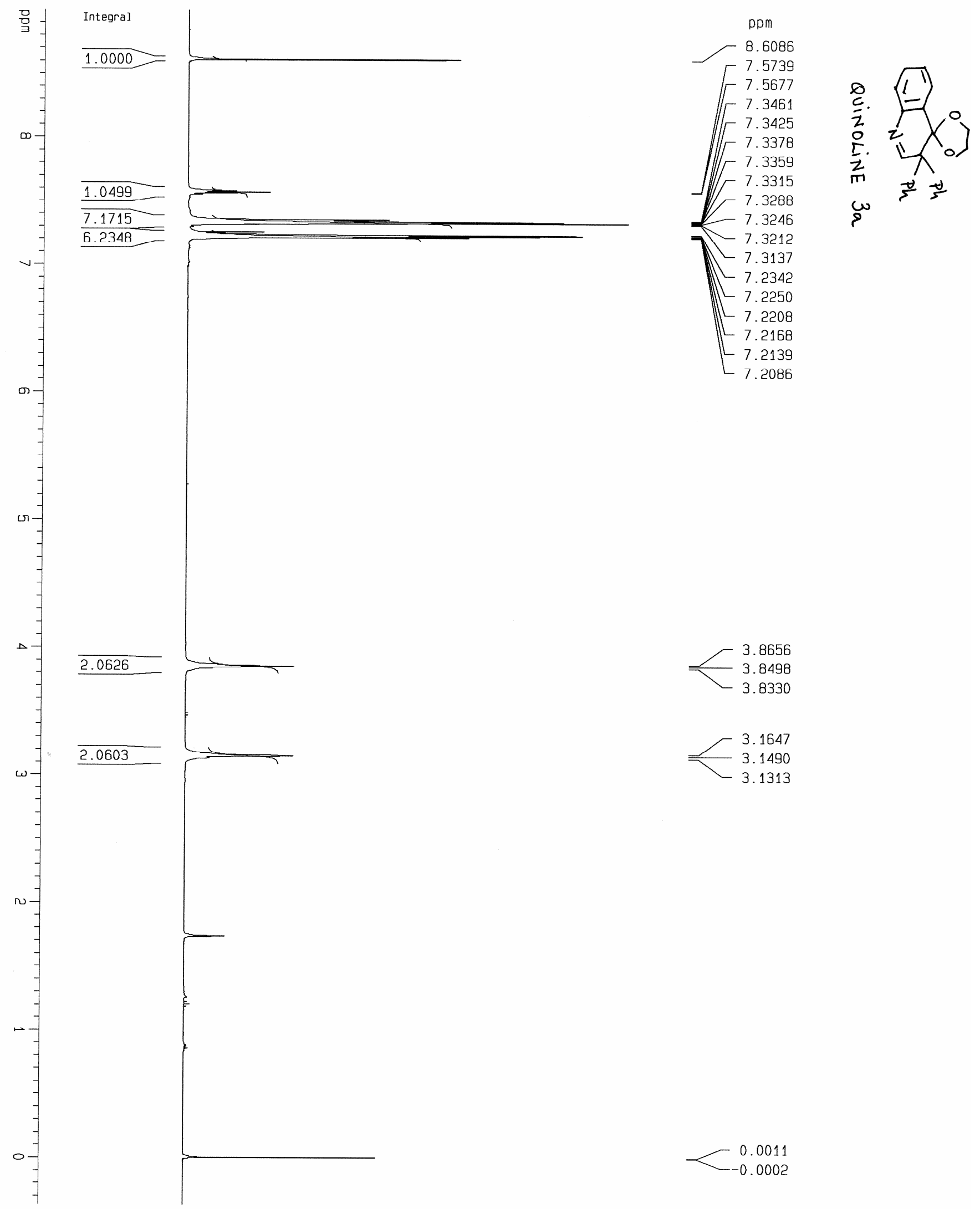



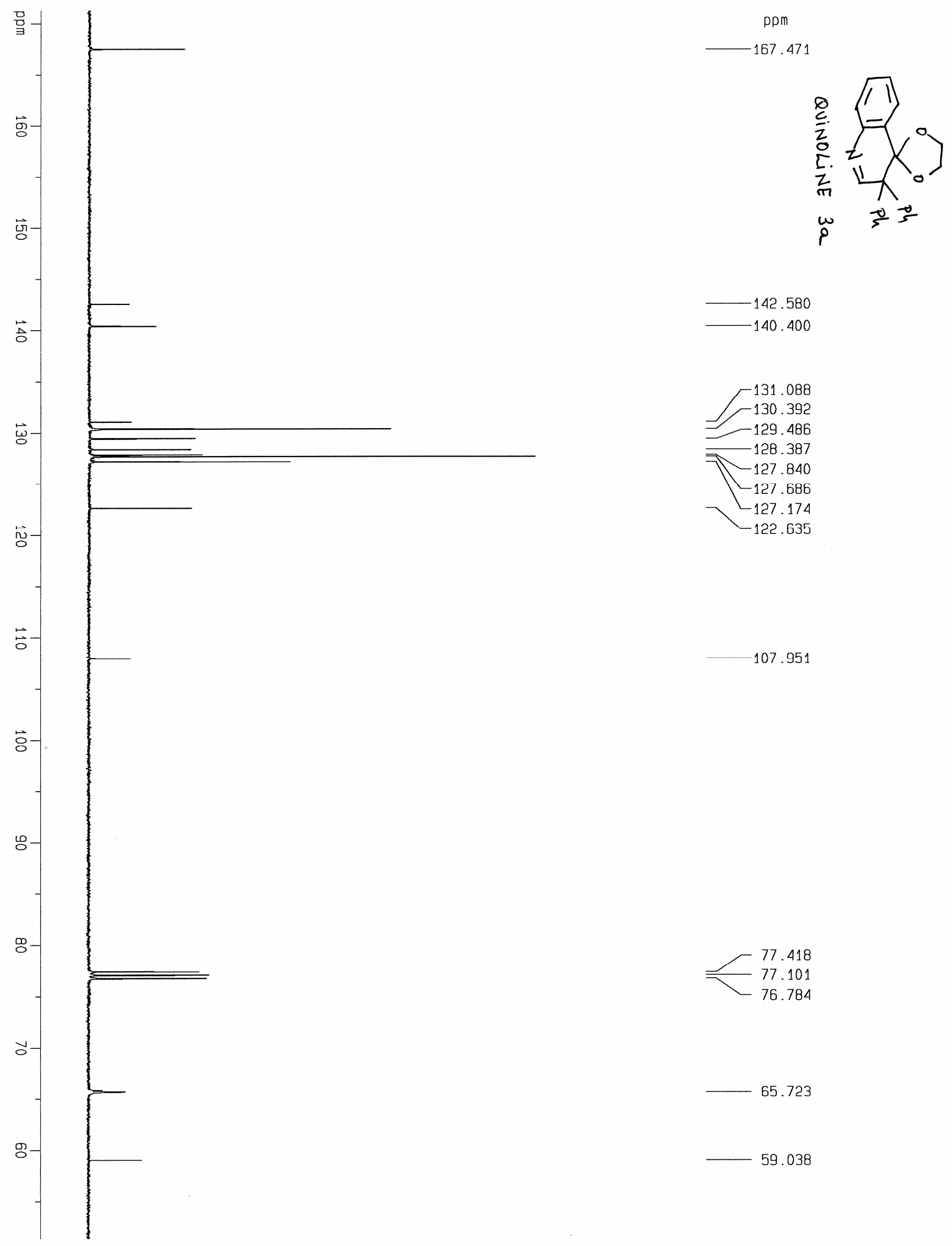

140.400

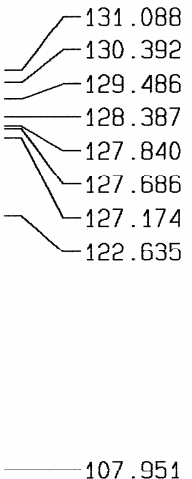




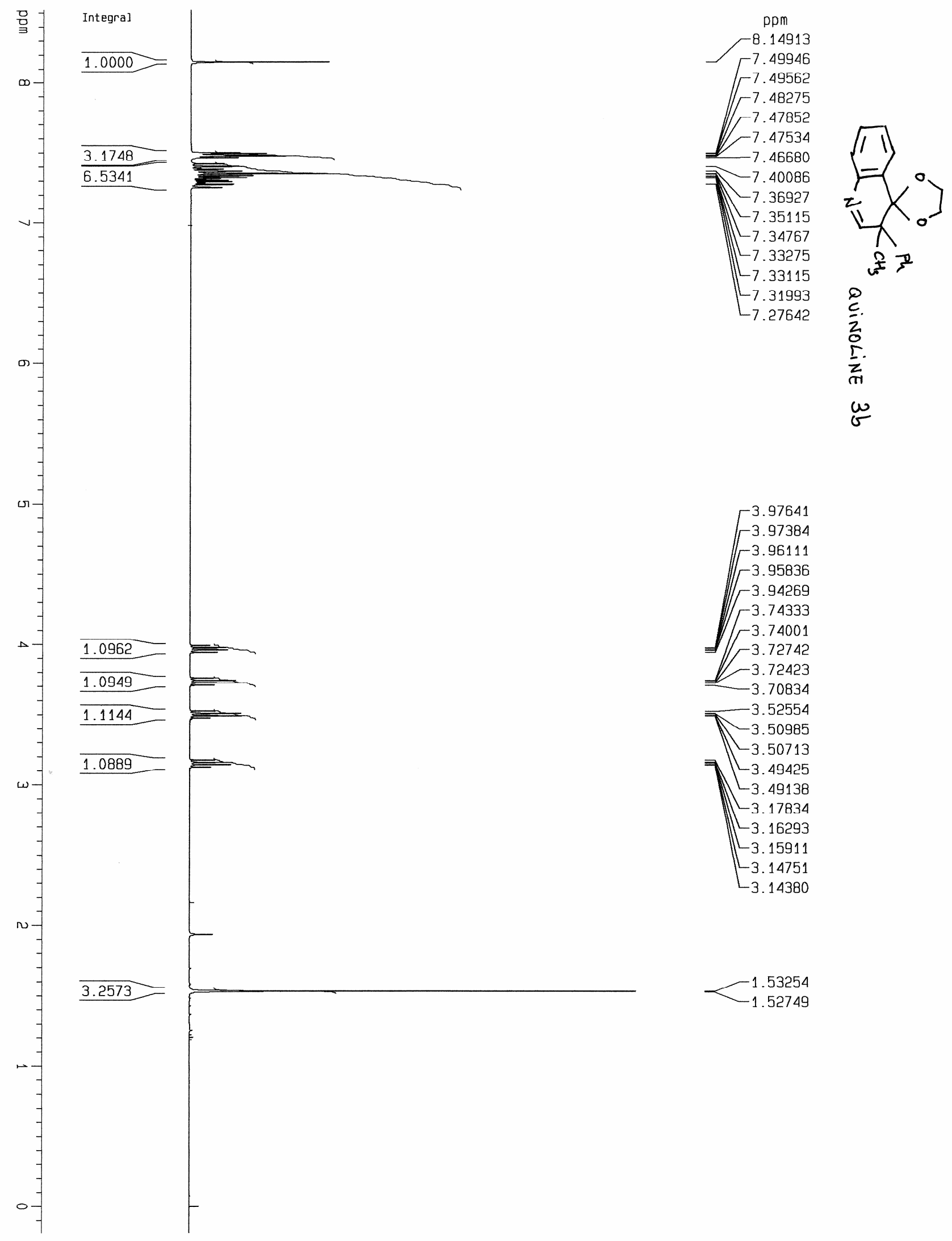




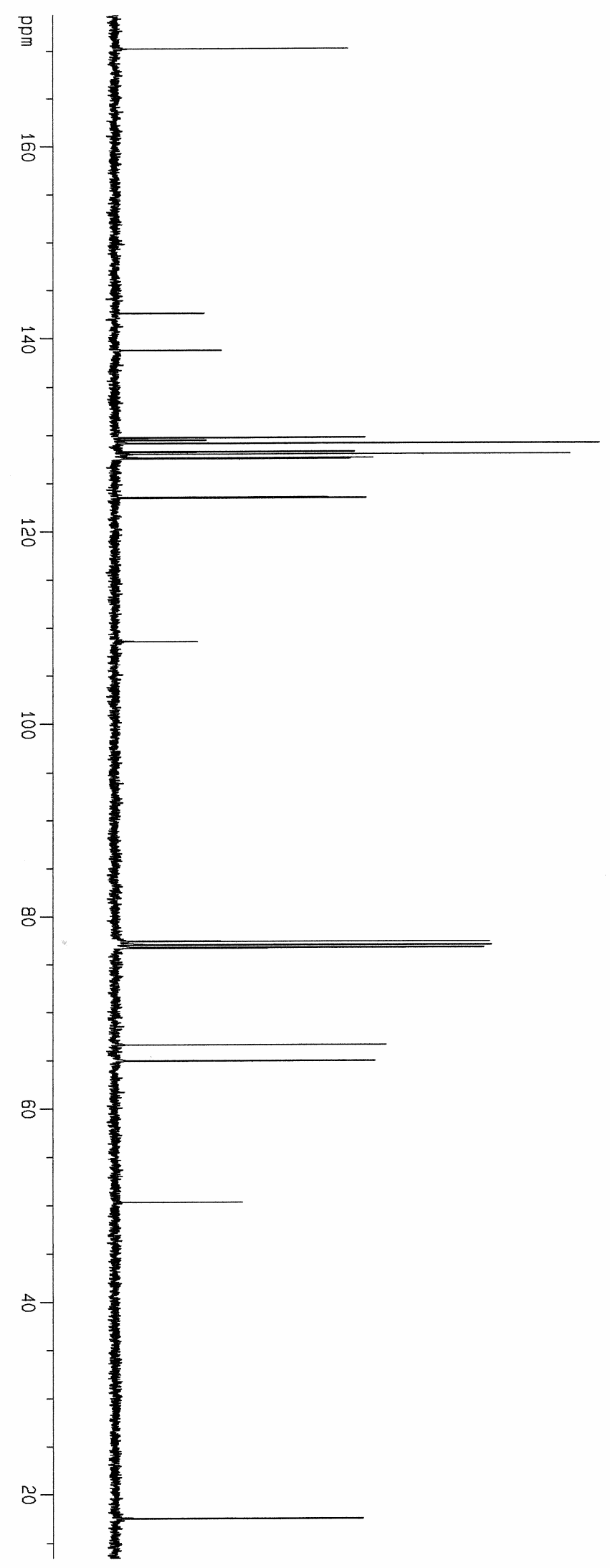



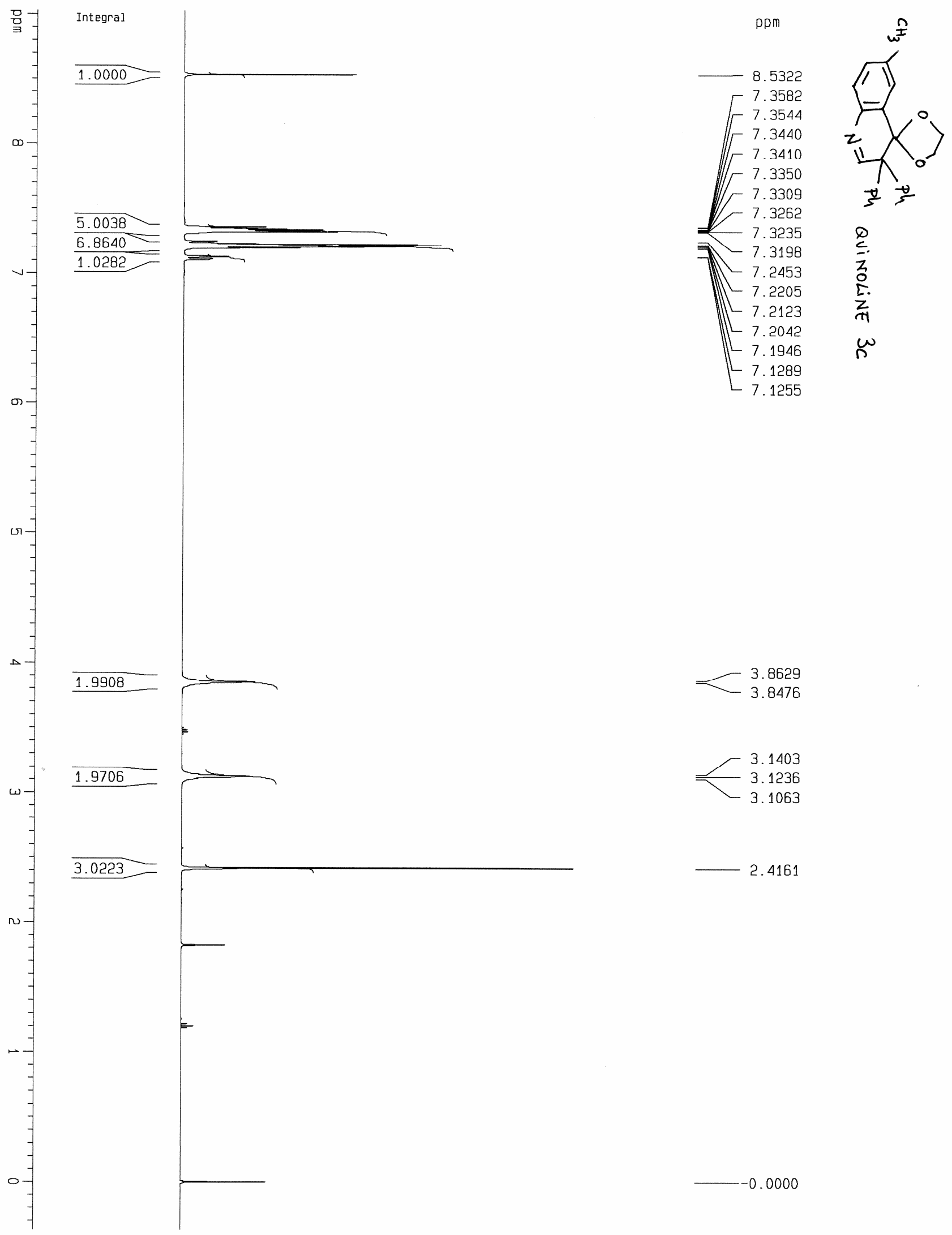

3.8476

3. 1403

-
$\square$
- .12366

2. 4161

$-0.0000$ 

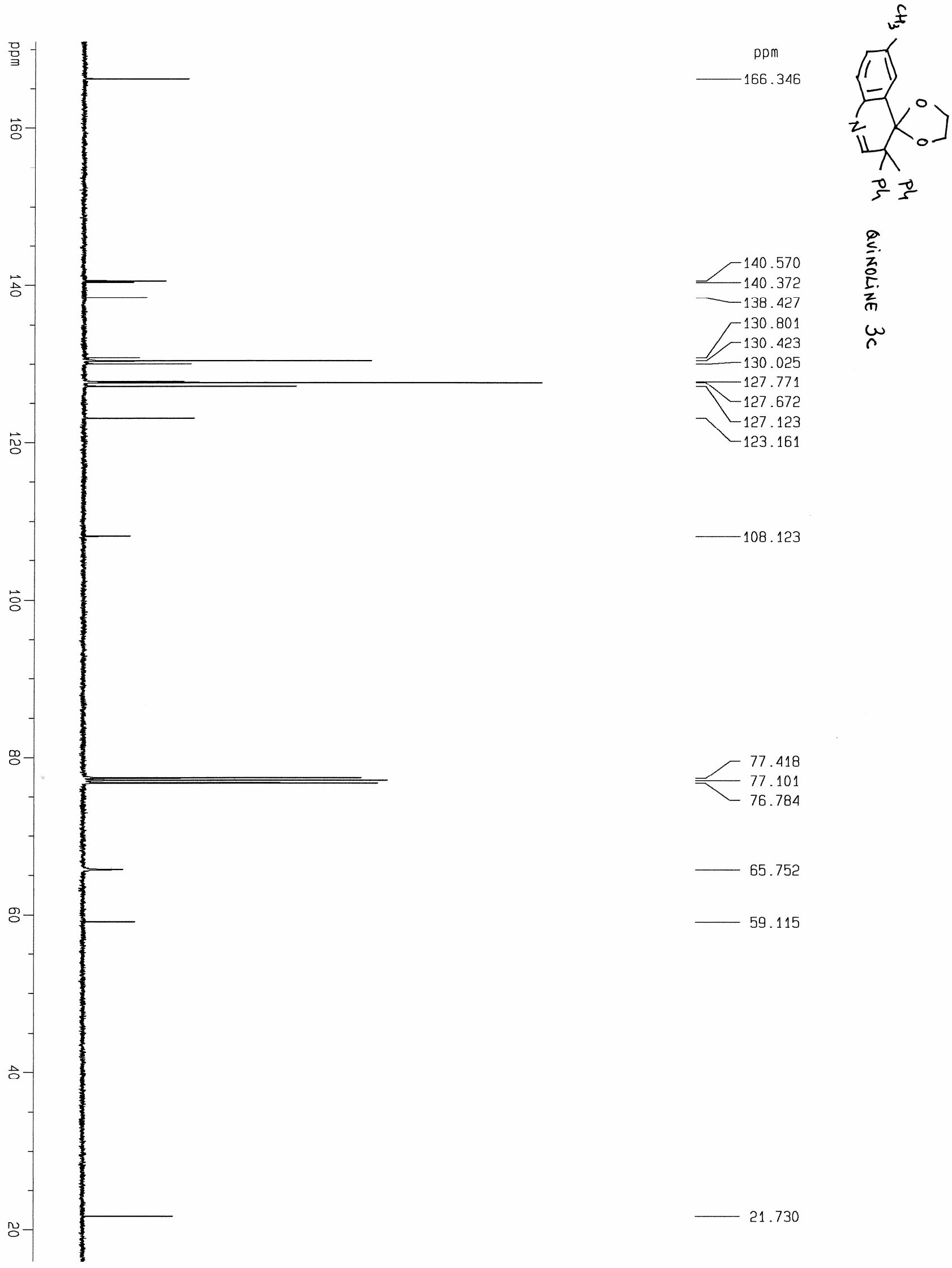

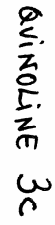

108.123

77.418

77.101

76.784

65.752

59.115

21.730 


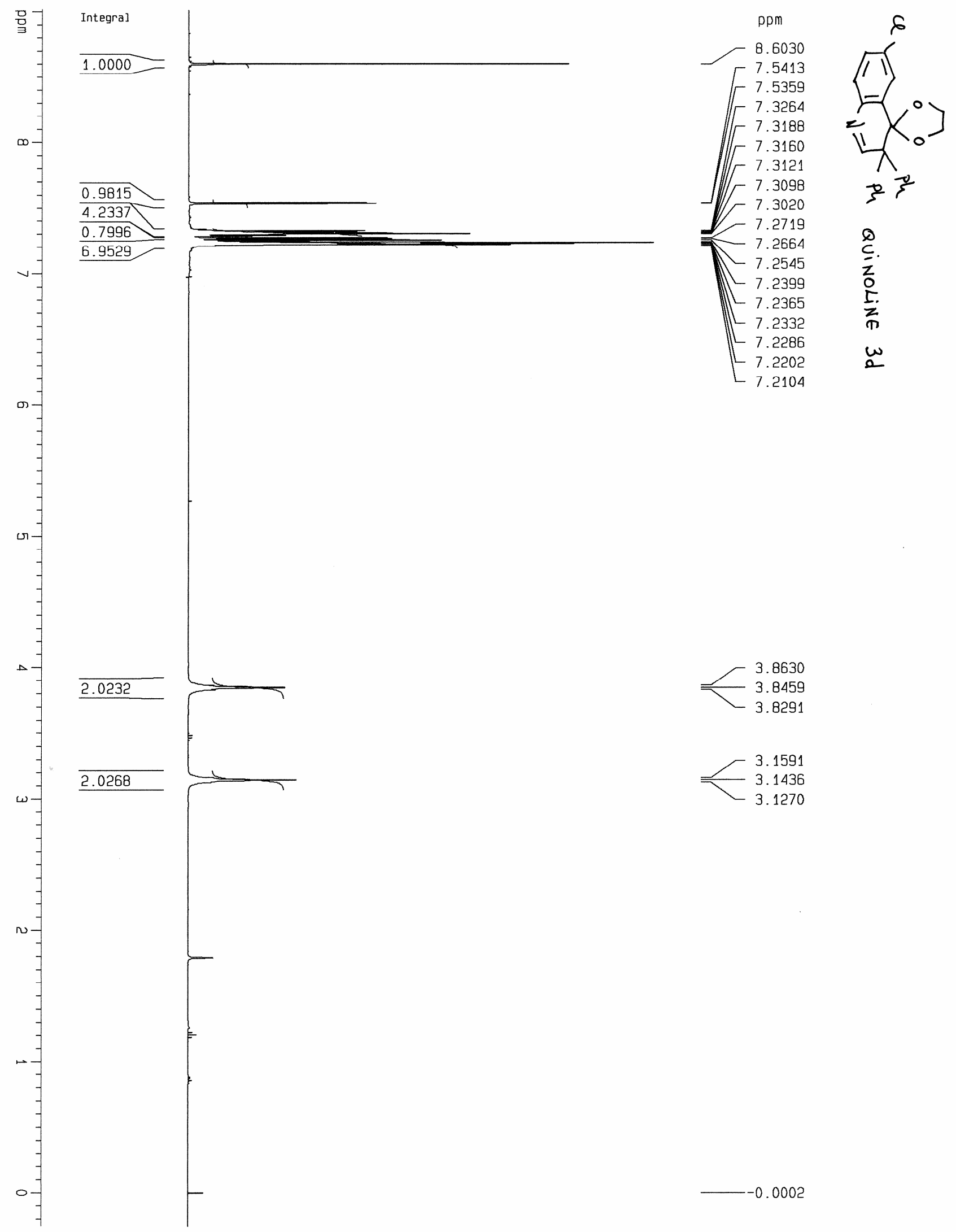



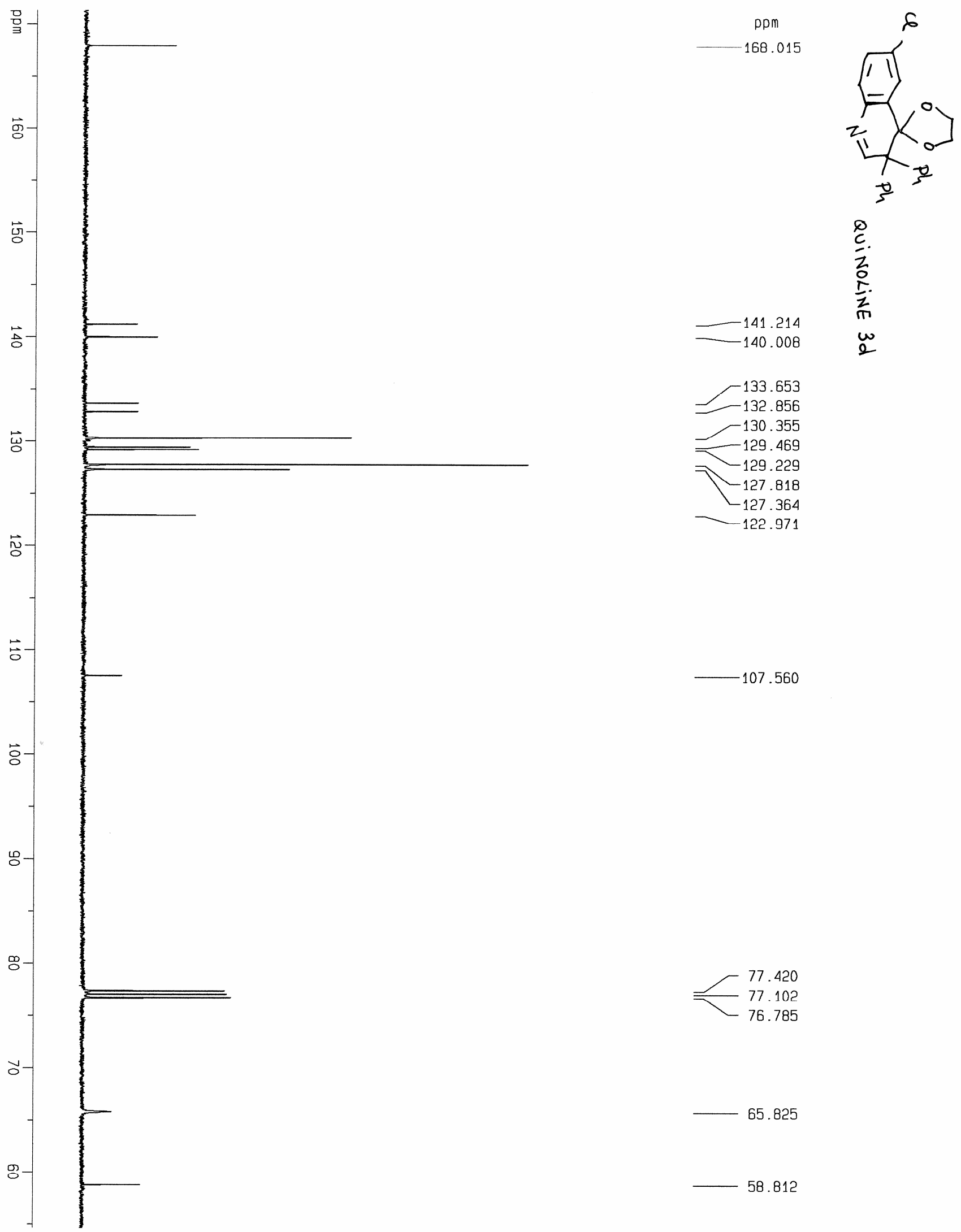

65.825

58.812 


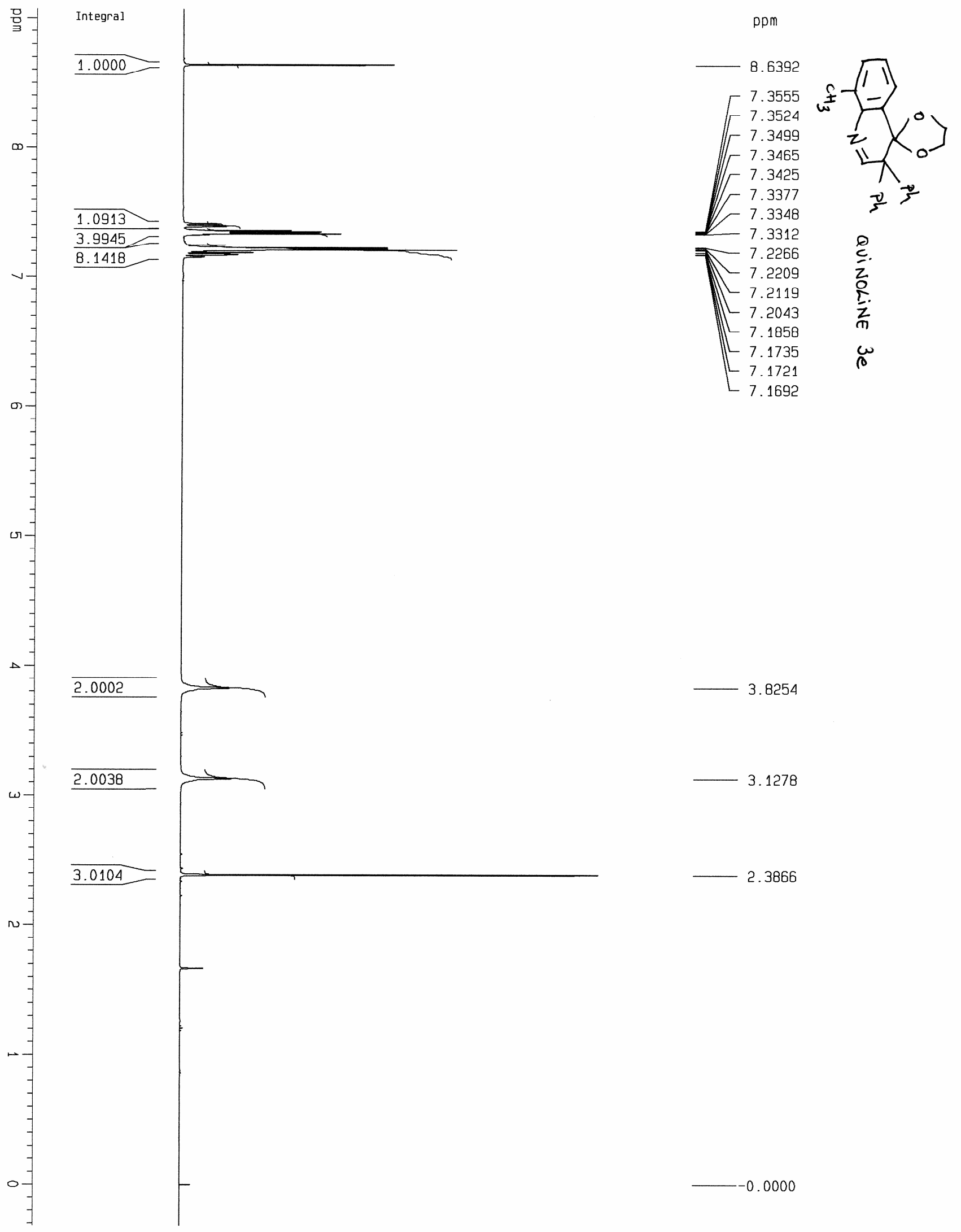




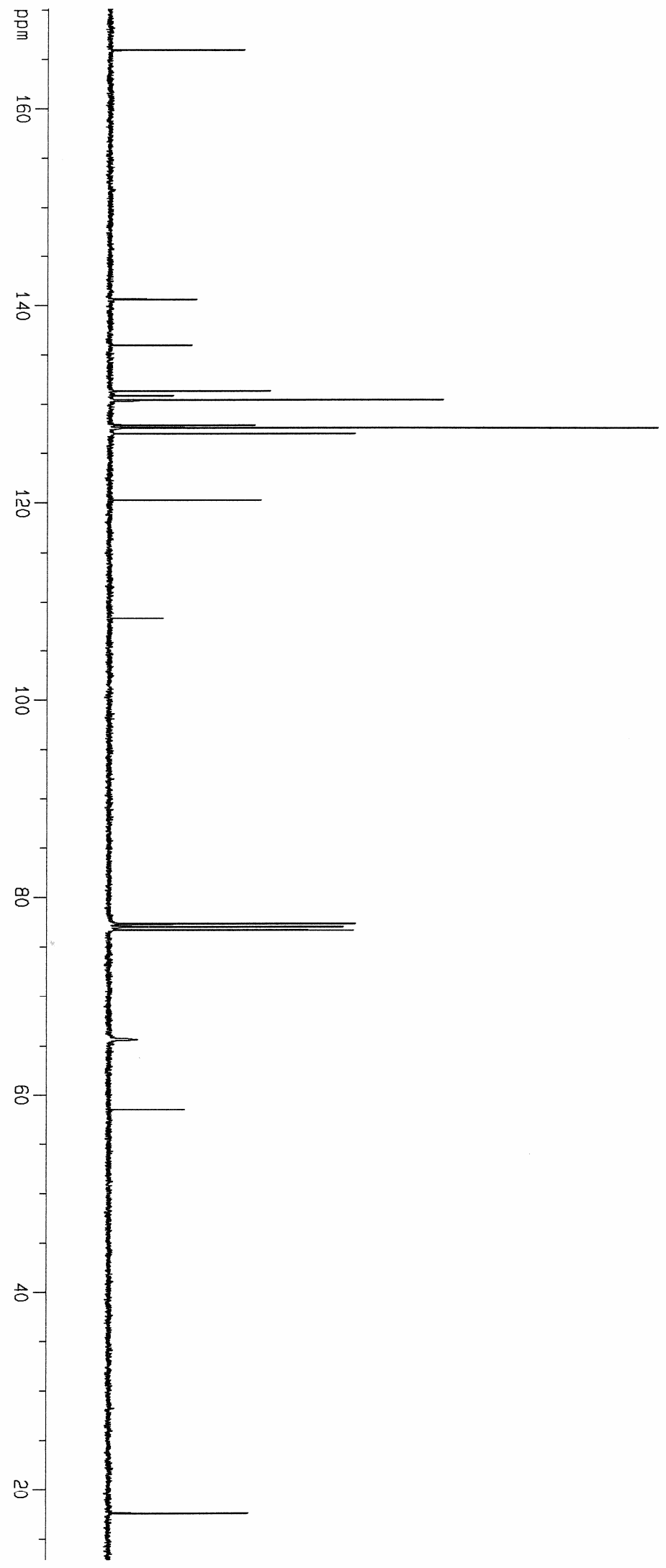

ppm

$-166.051$
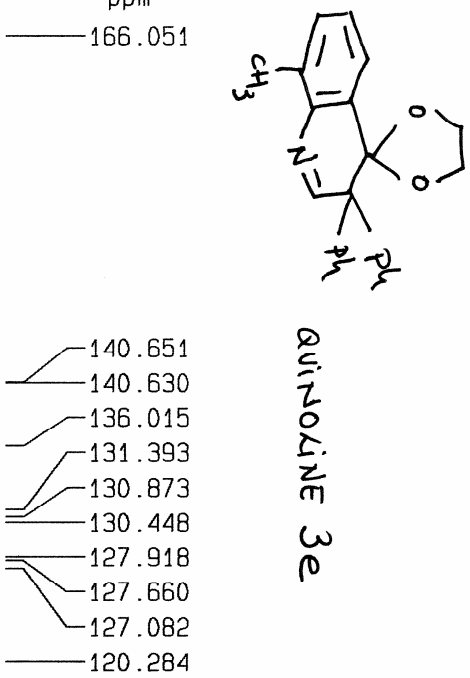

0
0
$i$
0
1.
$\vdots$
$\omega$
$\omega$
0

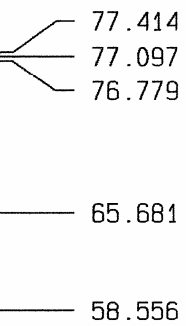

17.628 

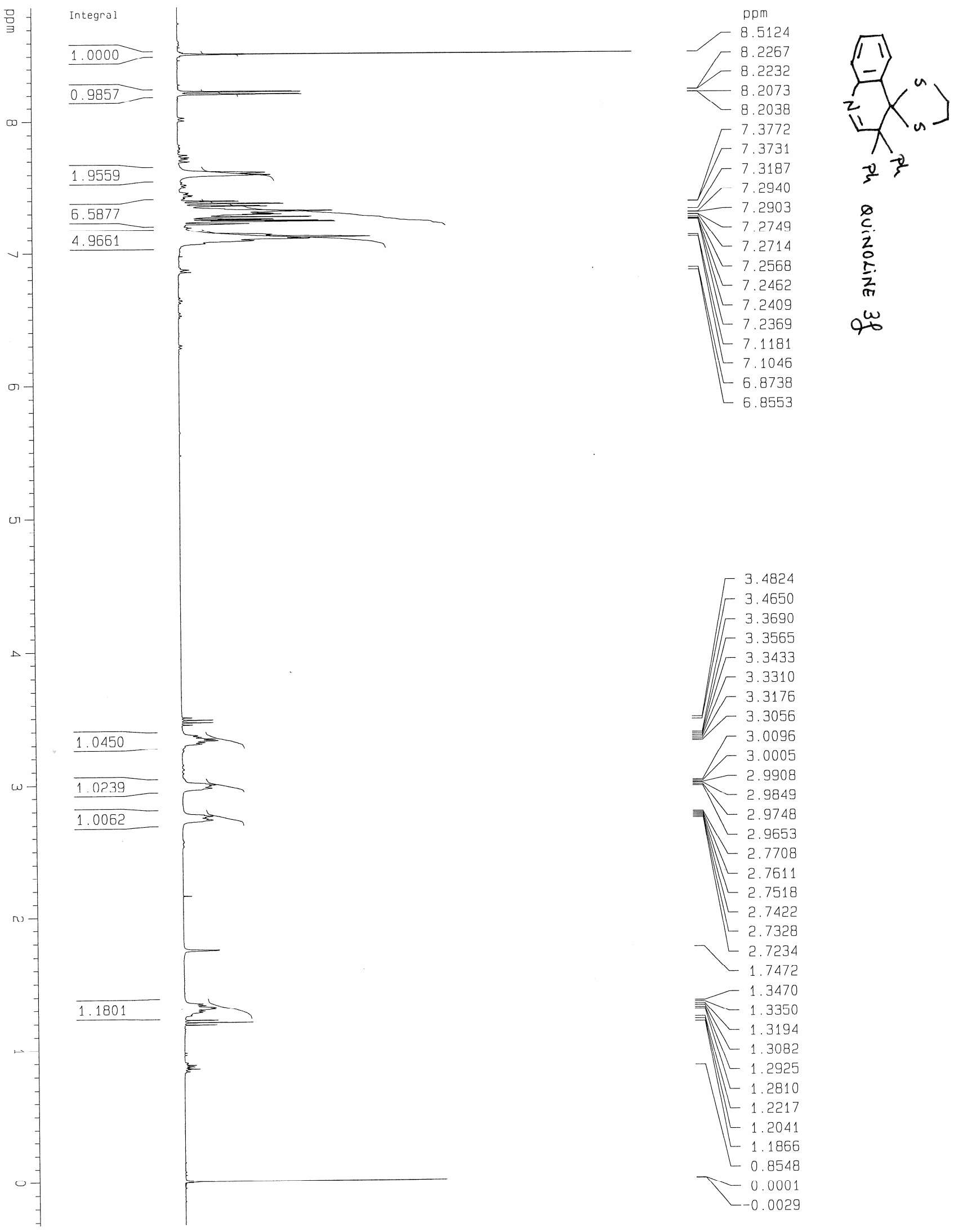

$\left[\begin{array}{r}3.4824 \\ -3.4650\end{array}\right.$

- 3.3690

- 3.3565

- 3.3433

F 3.3310

$\int 3.3176$

$=-3.3056$

F 3.0096

$-3.0005$

2.9908

$-2.9849$

$\lcm{2.9748}$

$\leftarrow 2.9653$

$L 2.7708$

$-2.7611$

$-2.7518$

L 2.7422

$\leftarrow 2.7328$

L 2.7234

$-1.7472$

1.3470

$-1.3350$

$\exists\lfloor 1.3194$

$L_{1} .3082$

I -1.2925

L 1.2810

$-1.2217$

L 1.2041

L 1.1866

L 0.8548

T 0.0001

$-0.0029$ 

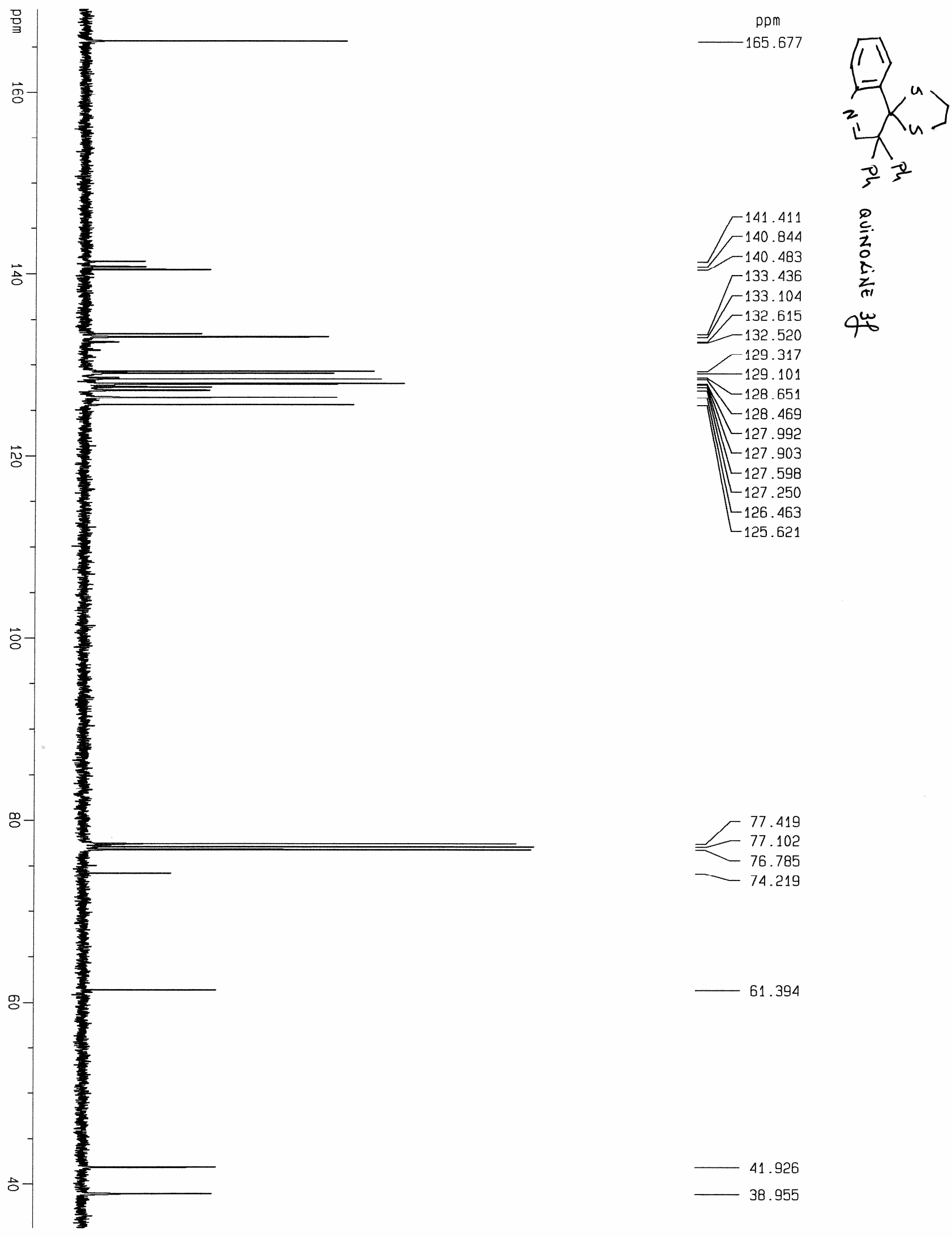

$-140.844$

$-140.483$

133.436

133.104

$-132.615$

$-132.520$

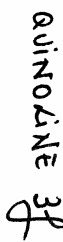

$-129.317$

$-129.101$

al工 -128.651

$-128.469$

$L_{127.992}$

$-127.903$

$L_{127.598}$

$-127.250$

$L_{126.463}$

$-125.621$

76.785

$-74.219$

61.394

- 41.926

38.955 


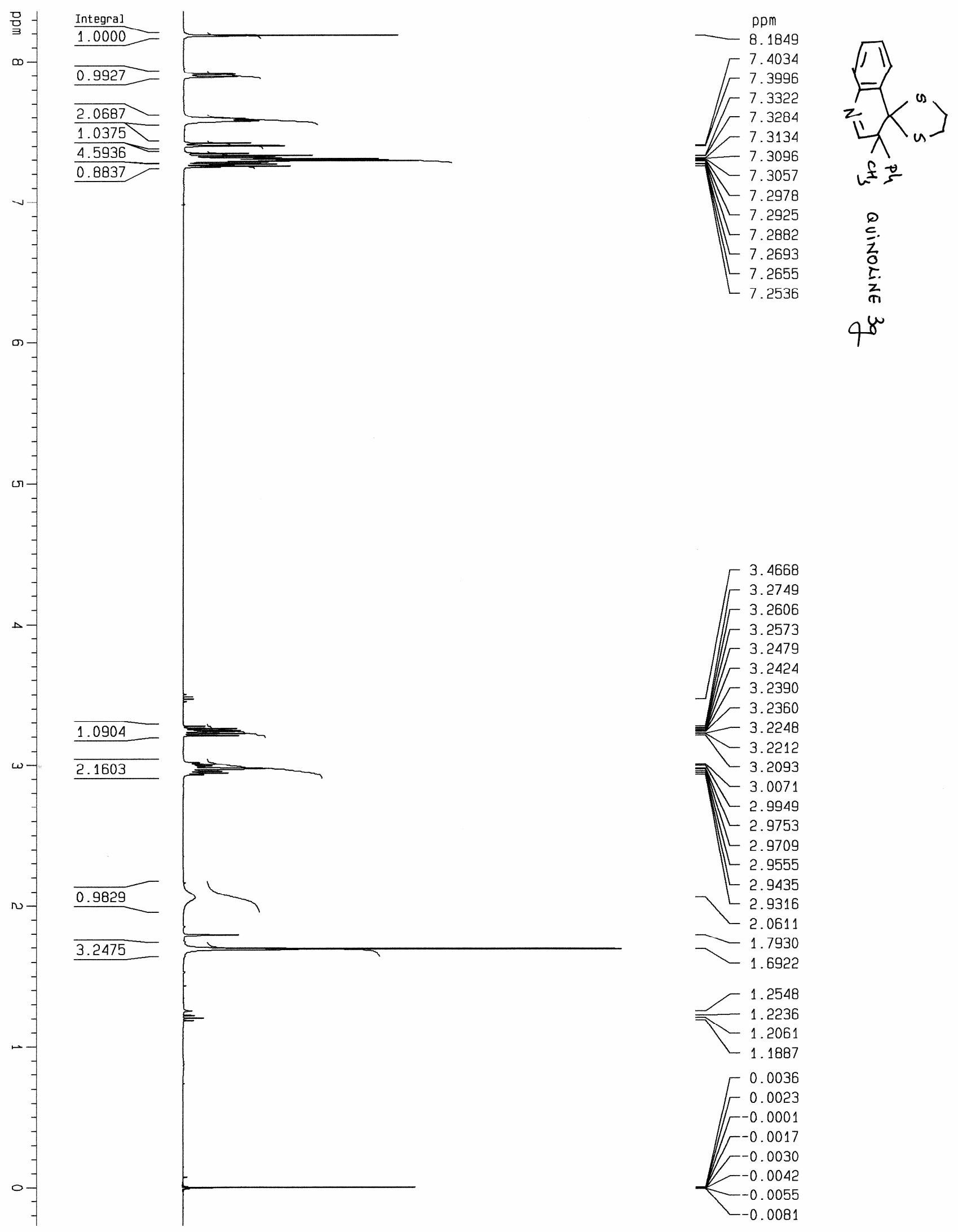



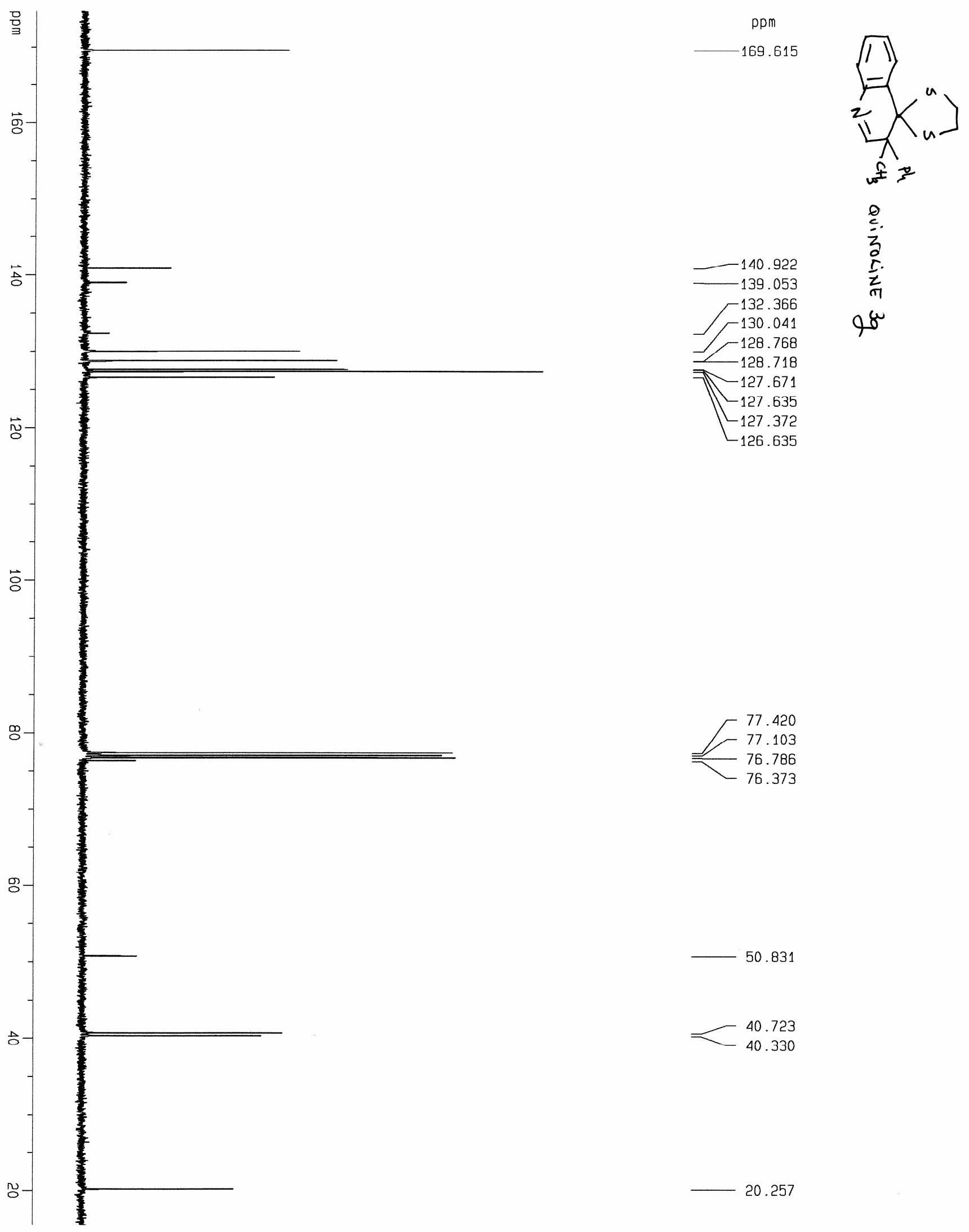

$+\quad 40.723$

40.330

20.257 

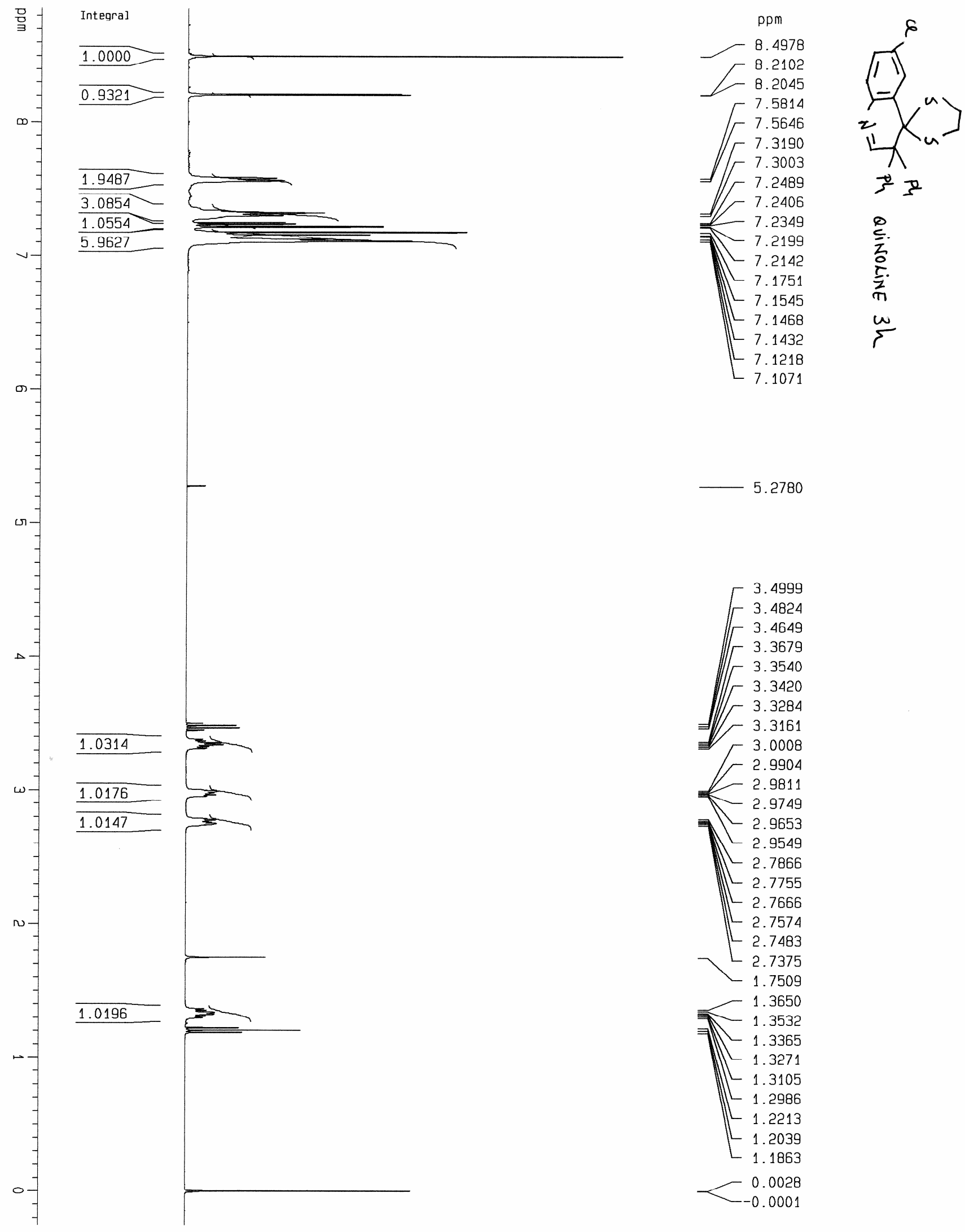

5. 2780

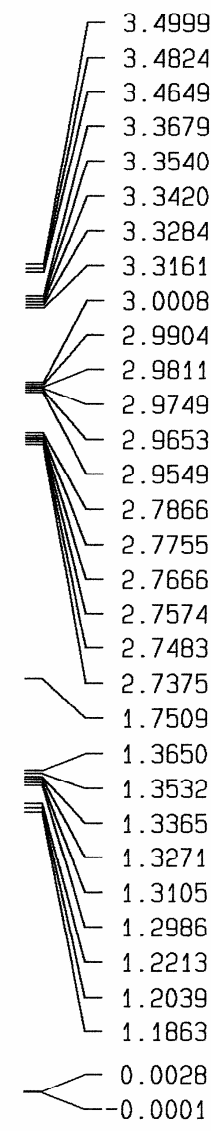



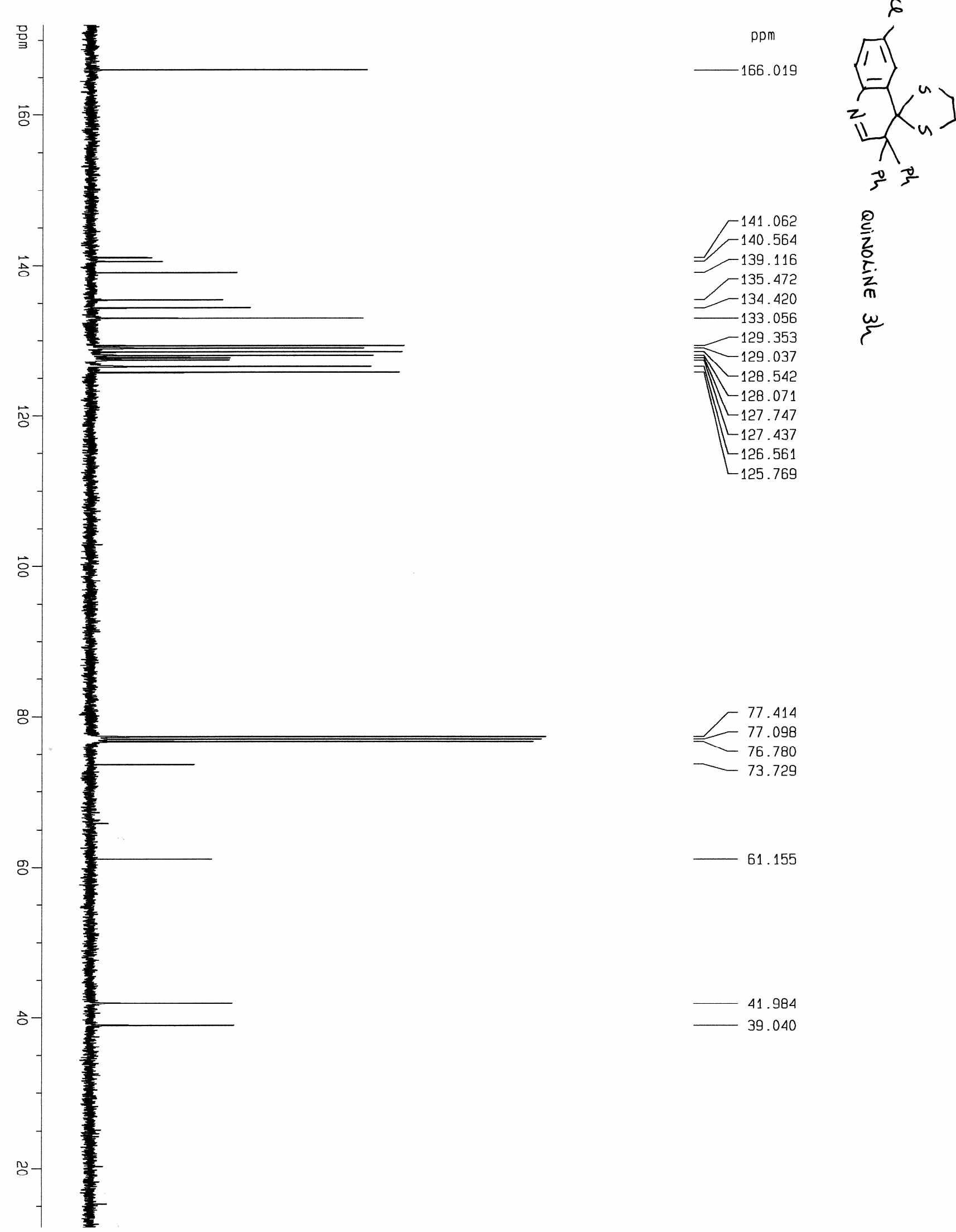

77.414

$-77.098$

$-76.780$

$-73.729$

61.155

41.984

39.040 


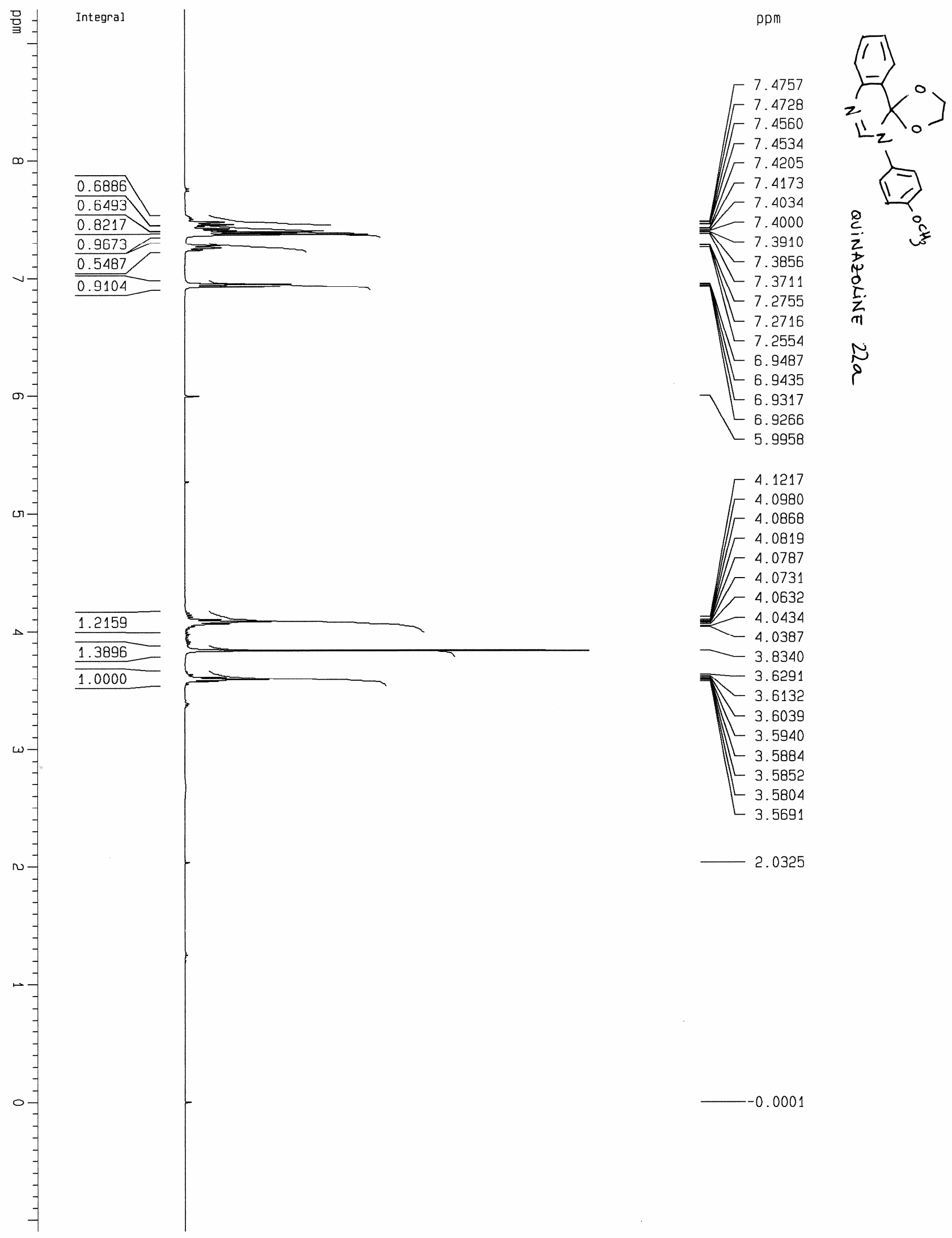



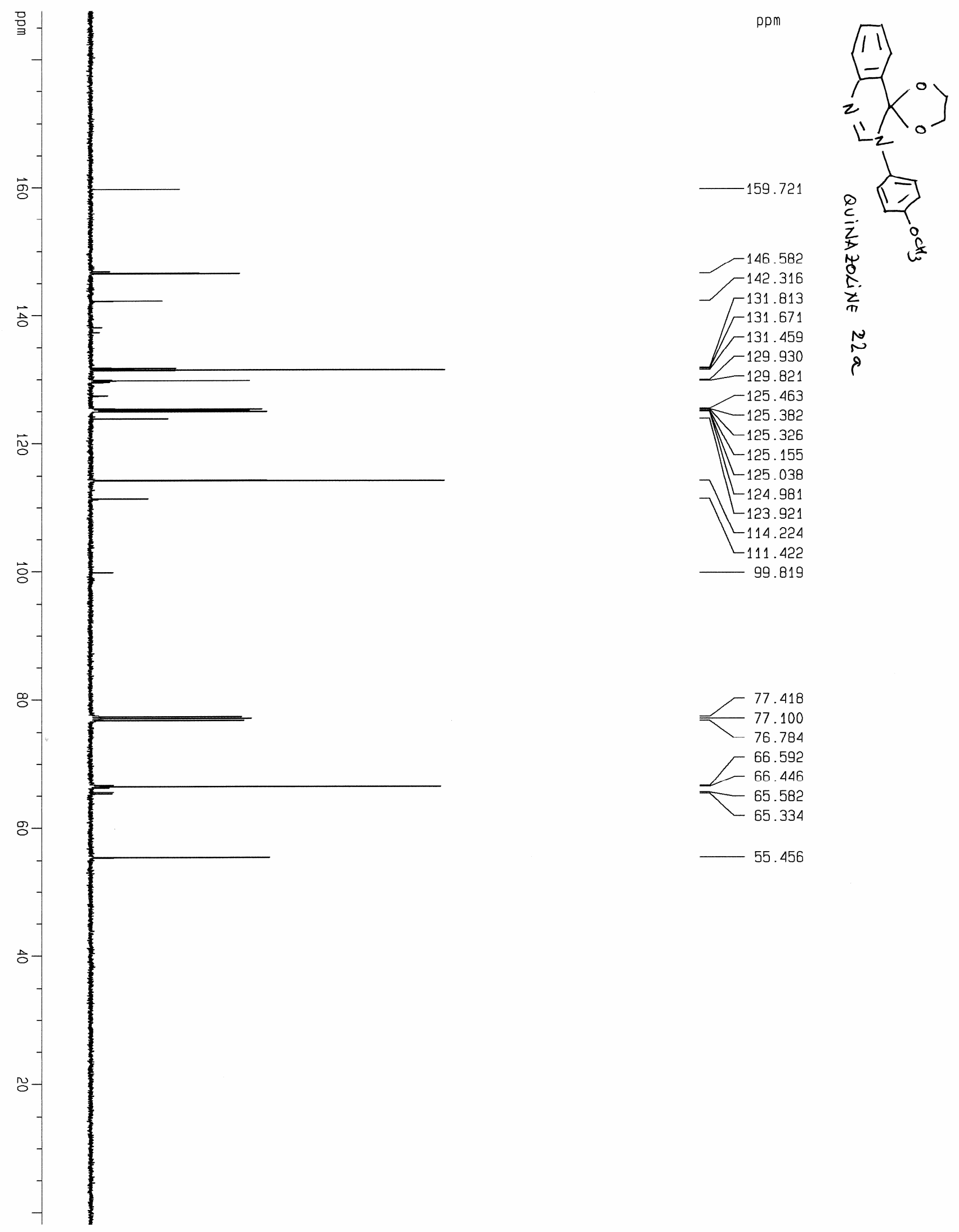

S44 


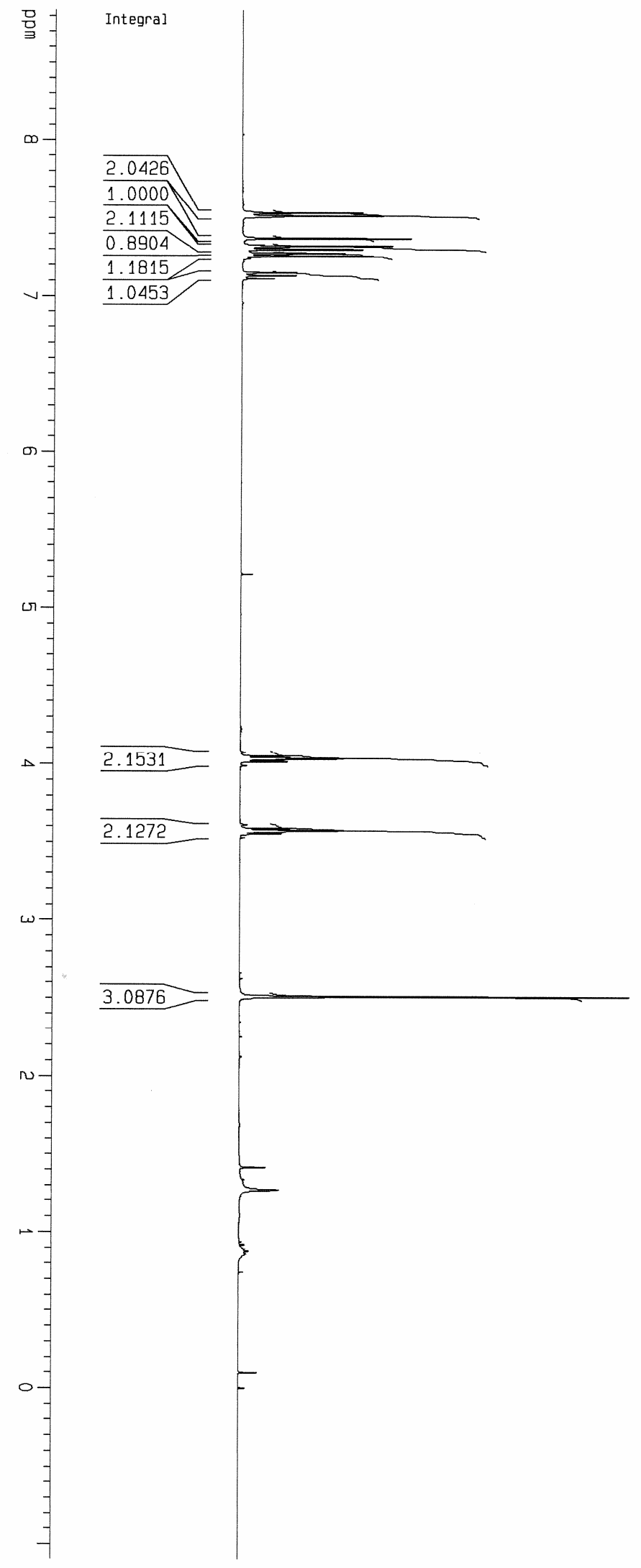




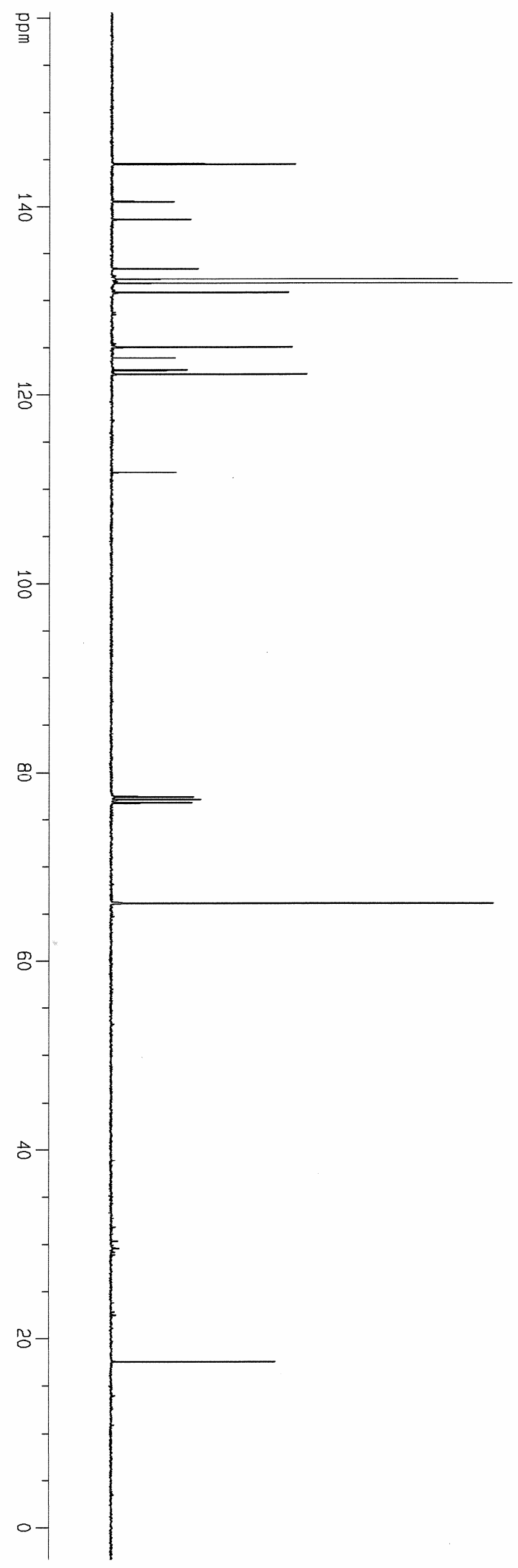

ppm

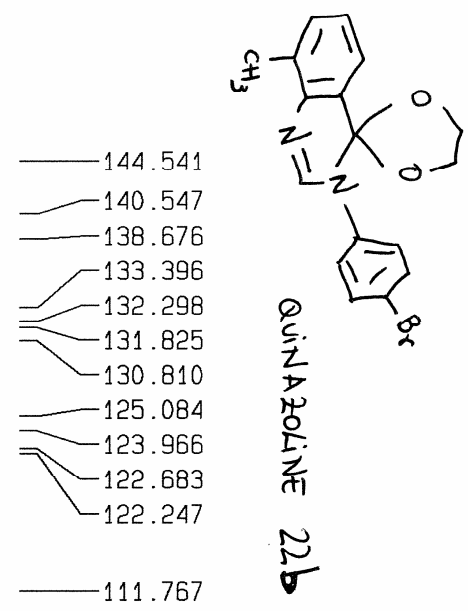

77.417

$-77.100$

$-76.782$

66. 167

17.594 

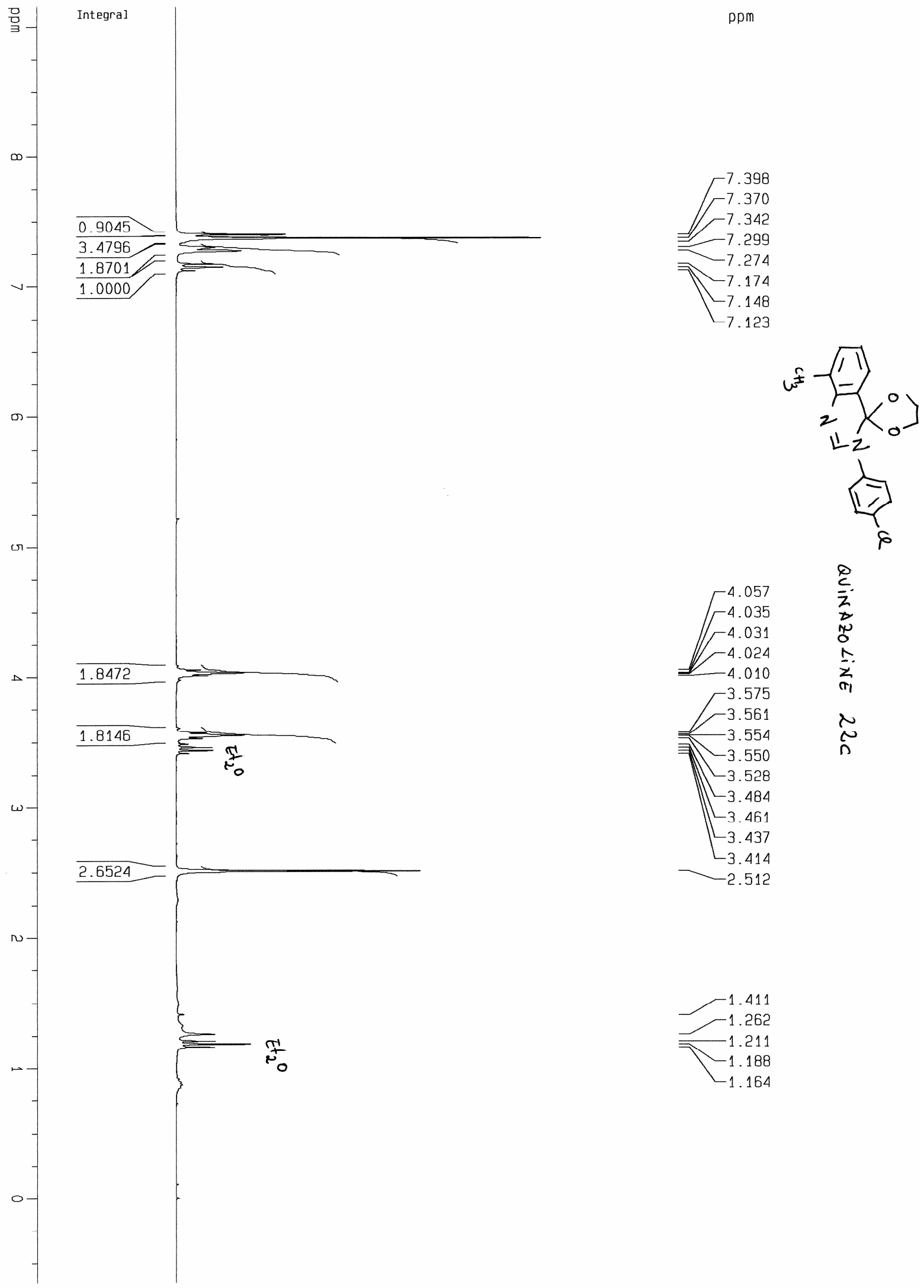

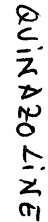

$-3.575$

$-3.561$

更 3.554

N

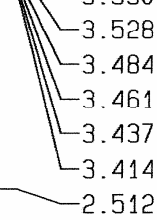

$-1.411$

$-1.262$

$-1.211$

$-1.188$

$-1.164$ 

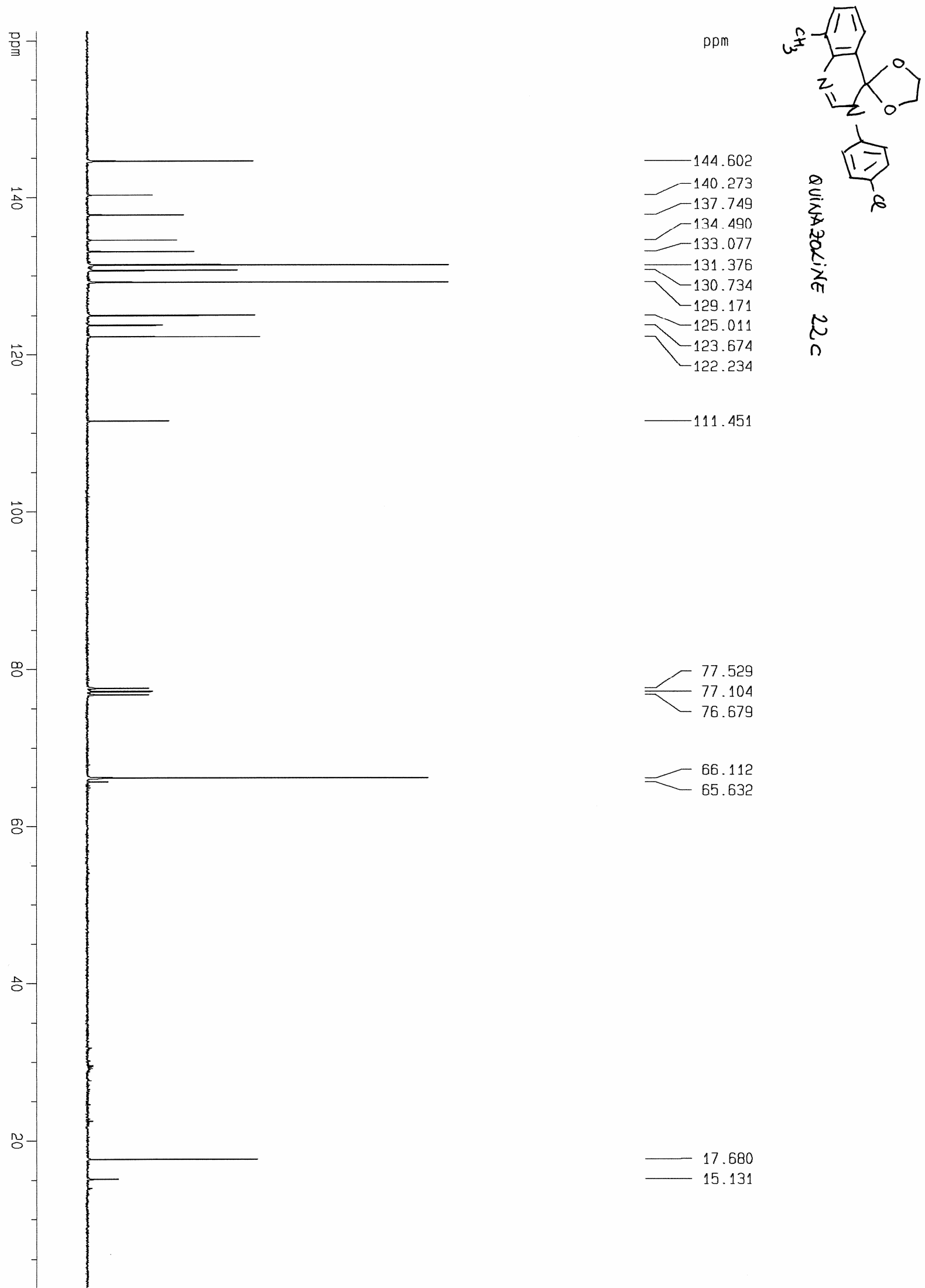

17.680

15. 131 


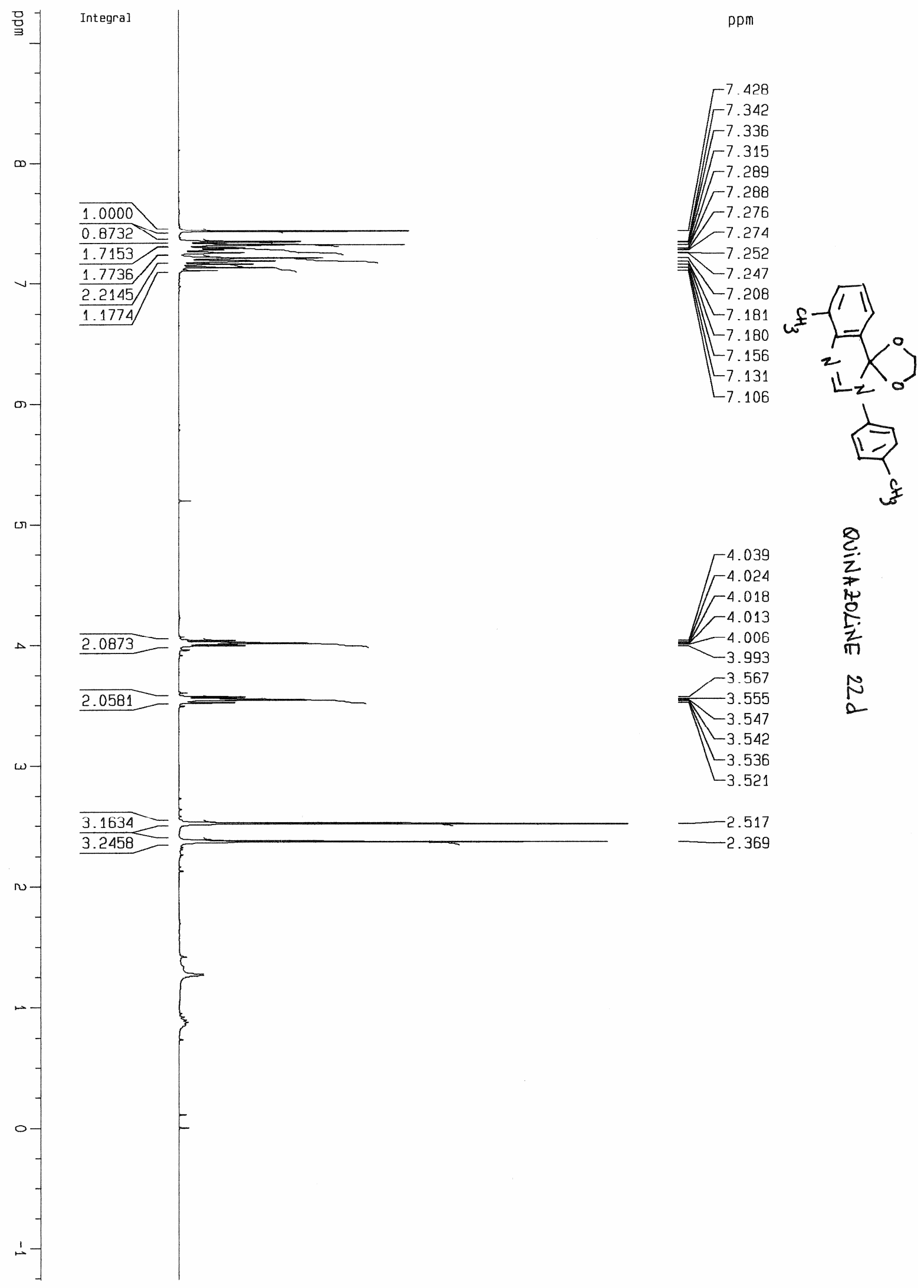



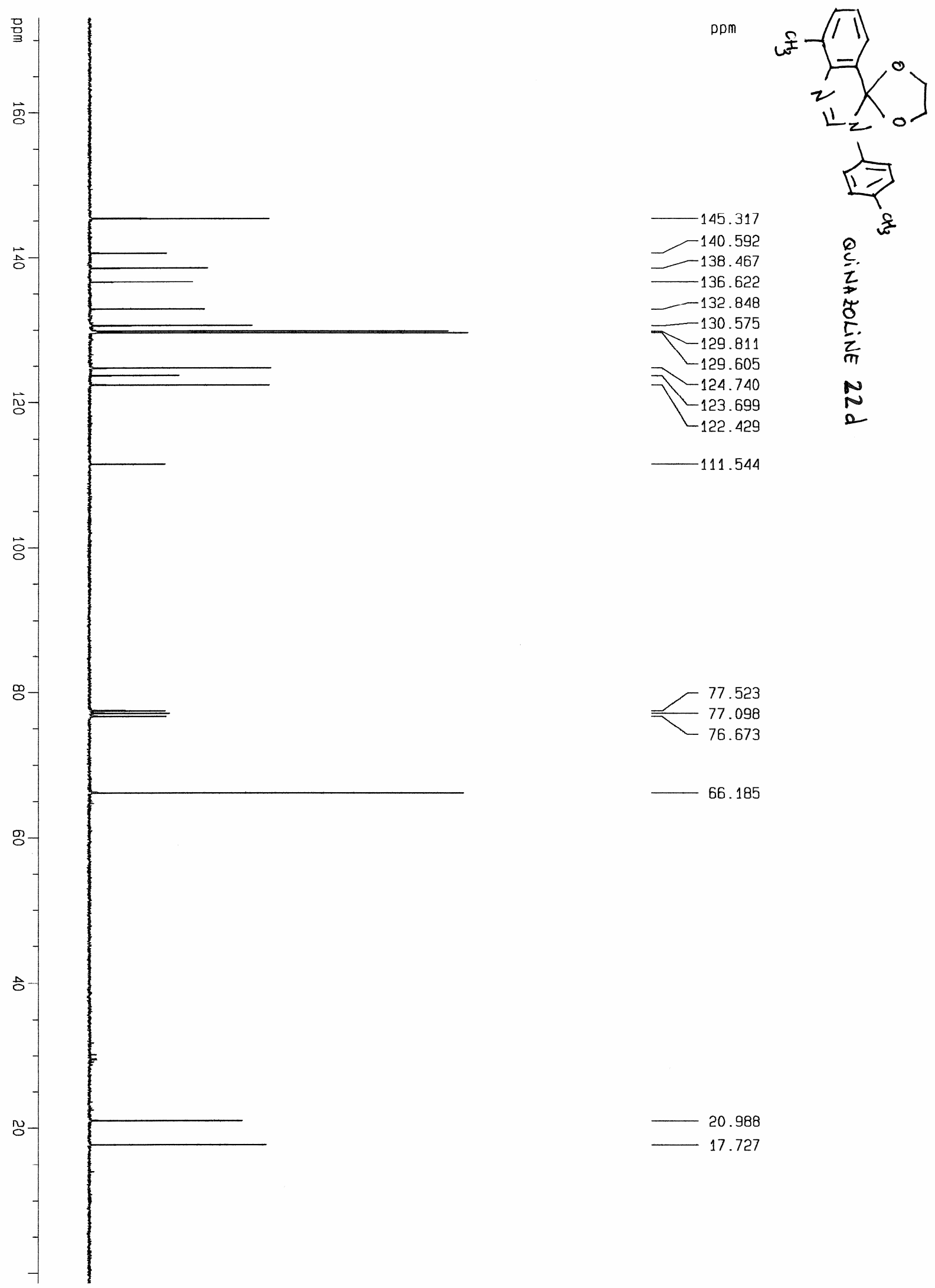

77.523

77.098

76.673

66. 185

20.988

17.727 

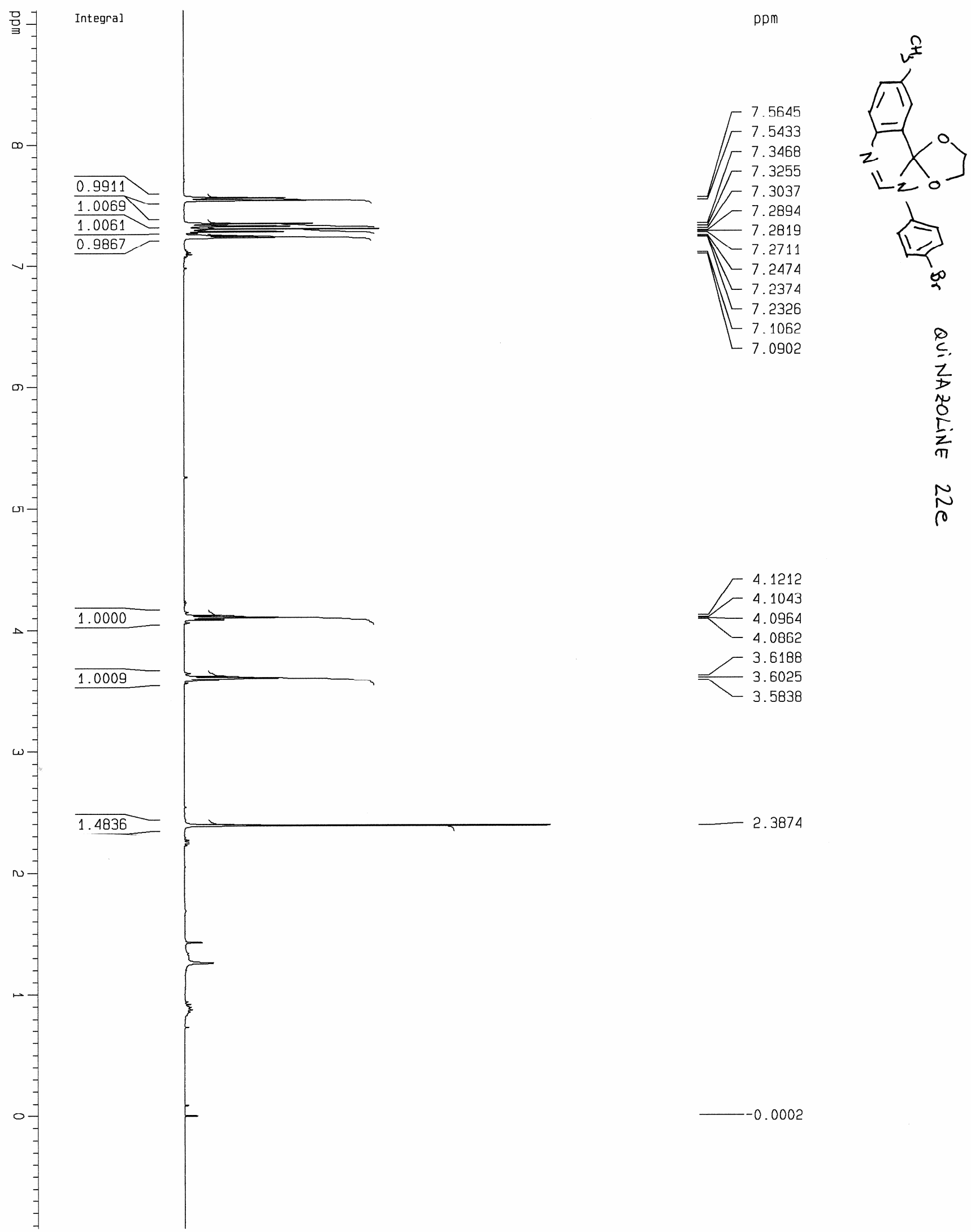

4. 1212

4.1043

4.0964

4.0862

3.6188

3.6025

- 3.5838

2. 3874

永

$\tilde{n}$



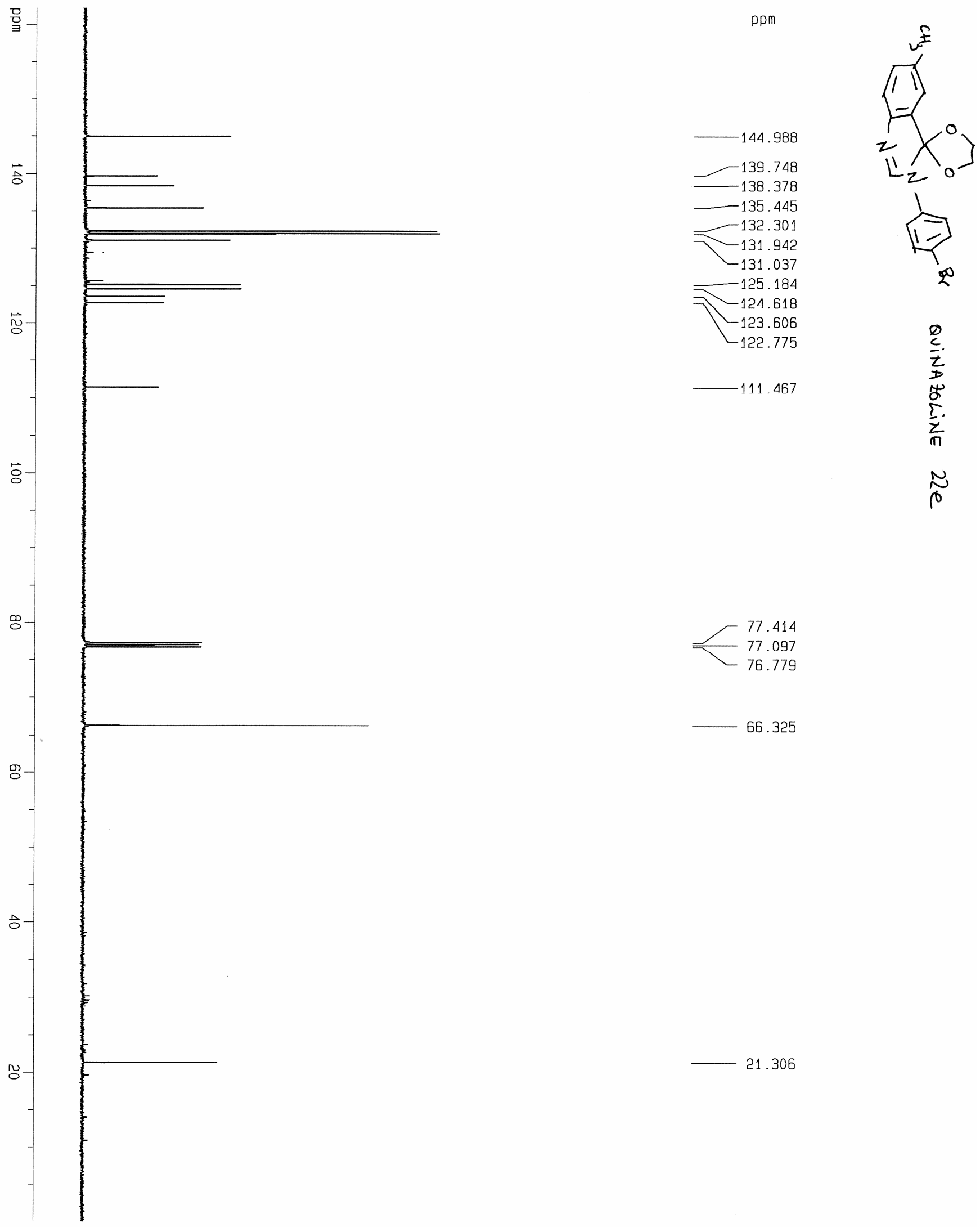

66.325

21.306 


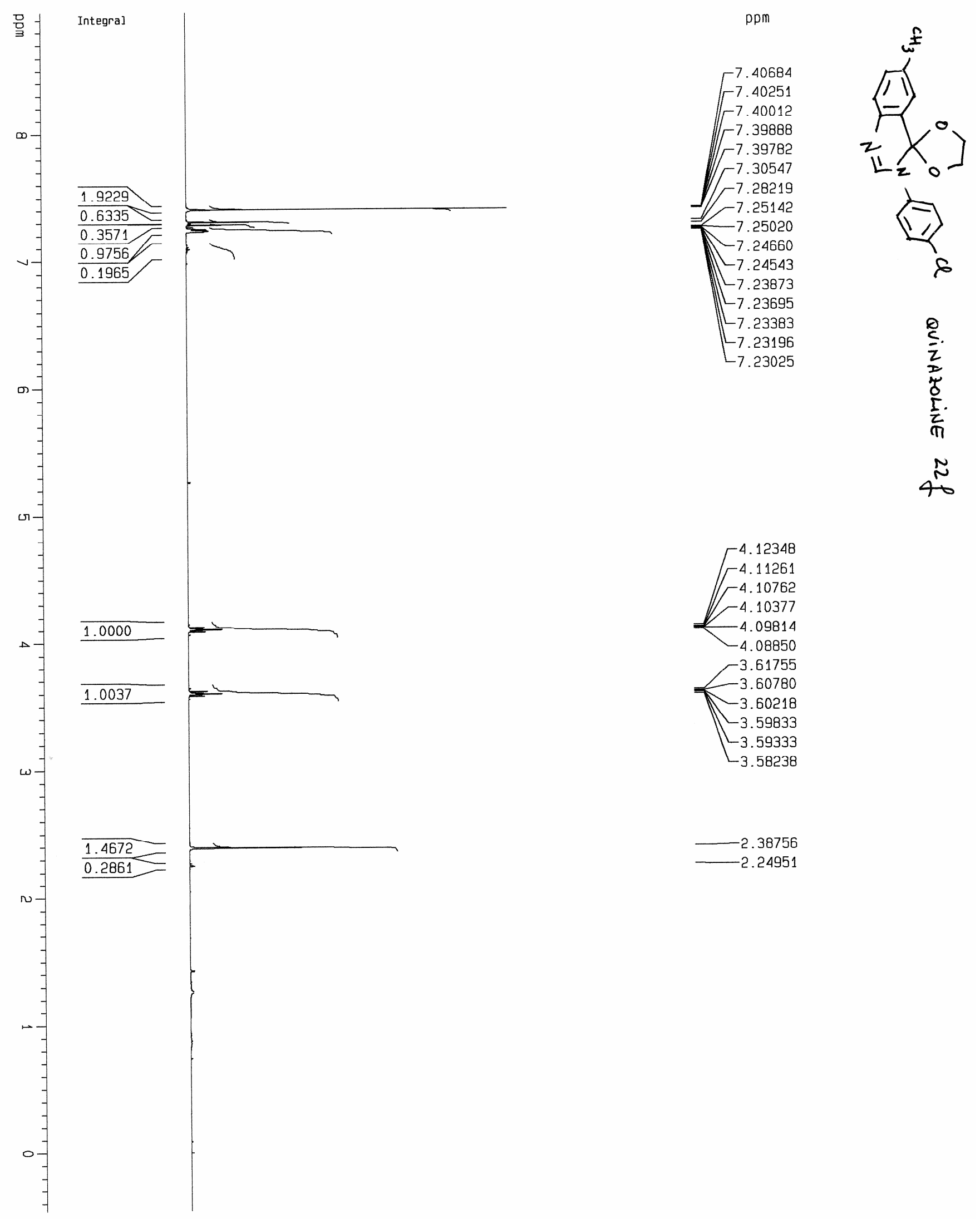



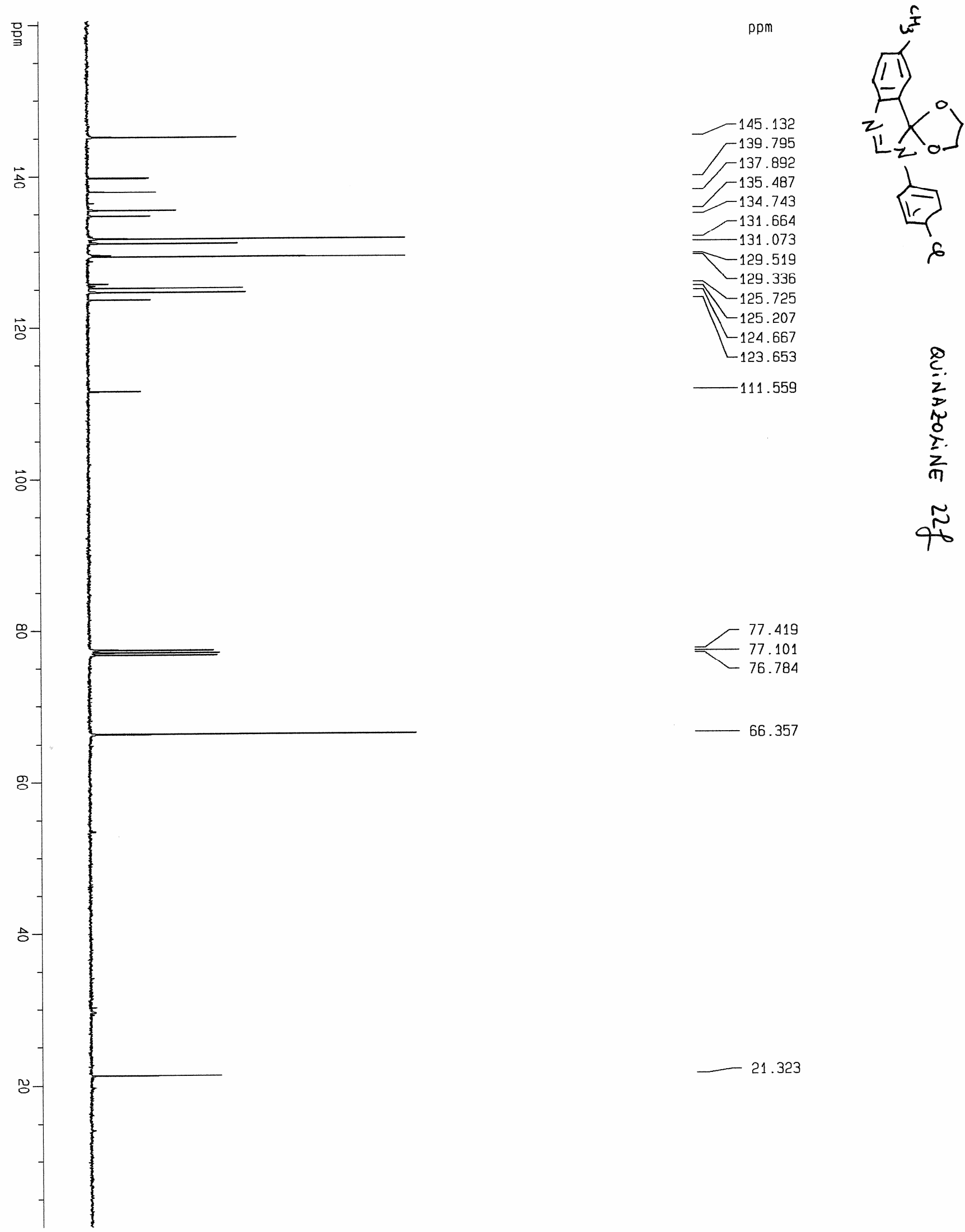

76.784
-761

66.357

21.323 